\title{
On Parametric Gevrey Asymptotics for Some Cauchy Problems in Quasiperiodic Function Spaces
}

\author{
A. Lastra ${ }^{1}$ and S. Malek ${ }^{2}$ \\ ${ }^{1}$ Departamento de Física y Matemáticas, University of Alcalá, Apartado de Correos 20, 28871 Alcalá de Henares, Spain \\ ${ }^{2}$ Laboratoire Paul Painlevé, University of Lille 1, 59655 Villeneuve d’Ascq Cedex, France
}

Correspondence should be addressed to A. Lastra; alberto.lastra@uah.es

Received 18 March 2014; Accepted 18 June 2014; Published 22 December 2014

Academic Editor: Graziano Crasta

Copyright (C) 2014 A. Lastra and S. Malek. This is an open access article distributed under the Creative Commons Attribution License, which permits unrestricted use, distribution, and reproduction in any medium, provided the original work is properly cited.

We investigate Gevrey asymptotics for solutions to nonlinear parameter depending Cauchy problems with $2 \pi$-periodic coefficients, for initial data living in a space of quasiperiodic functions. By means of the Borel-Laplace summation procedure, we construct sectorial holomorphic solutions which are shown to share the same formal power series as asymptotic expansion in the perturbation parameter. We observe a small divisor phenomenon which emerges from the quasiperiodic nature of the solutions space and which is the origin of the Gevrey type divergence of this formal series. Our result rests on the classical Ramis-Sibuya theorem which asks to prove that the difference of any two neighboring constructed solutions satisfies some exponential decay. This is done by an asymptotic study of a Dirichlet-like series whose exponents are positive real numbers which accumulate to the origin.

\section{Introduction}

We consider a family of nonlinear Cauchy problems of the form

$$
\begin{aligned}
\left(\epsilon^{r_{3}}\left(t^{2} \partial_{t}+t\right)^{r_{2}}+\left(-i \partial_{z}+1\right)^{r_{1}}\right) \partial_{x}^{S} X_{i}(t, z, x, \epsilon) & \\
= & \sum_{\underline{k}=\left(s, k_{0}, k_{1}, k_{2}\right) \in \mathcal{S}} b_{k}(z, x, \epsilon) t^{s} \partial_{t}^{k_{0}} \partial_{z}^{k_{1}} \partial_{x}^{k_{2}} X_{i}(t, z, x, \epsilon) \\
& +\sum_{\underline{l}=\left(l_{0}, l_{1}\right) \in \mathcal{N}} \mathcal{c}_{l}(z, x, \epsilon) t^{l_{0}+l_{1}-1}\left(X_{i}(t, z, x, \epsilon)\right)^{l_{1}}
\end{aligned}
$$

for given initial data

$$
\begin{gathered}
\left(\partial_{x}^{j} X_{i}\right)(t, z, 0, \epsilon)=\Xi_{i, j}(t, z, \epsilon), \\
0 \leq i \leq v-1, \quad 0 \leq j \leq S-1,
\end{gathered}
$$

where $\epsilon$ is a complex parameter, $S, r_{1}, r_{2}, r_{3}$ are some positive integers, $\mathcal{S}$ is a finite subset of $\mathbb{N}^{4}$, and $\mathcal{N}$ is a finite subset of $\mathbb{N}^{2}$ that fulfills constraints (185). The coefficients $b_{\underline{k}}(z, x, \epsilon)$ of the linear part and $c_{l}(z, x, \epsilon)$ of the nonlinear part are $2 \pi$ periodic Fourier series

$$
\begin{gathered}
b_{\underline{k}}(z, x, \epsilon)=\sum_{\beta \geq 0} b_{\underline{k}, \beta}(x, \epsilon) e^{i z \beta}, \\
c_{\underline{l}}(z, x, \epsilon)=\sum_{\beta \geq 0} c_{\underline{l}, \beta}(x, \epsilon) e^{i z \beta}
\end{gathered}
$$

in the variable $z$ with coefficients $b_{k, \beta}(x, \epsilon), c_{l, \beta}(x, \epsilon)$ in $\mathcal{O}\{x, \epsilon\}$ (which denotes the Banach space of bounded holomorphic functions in $(x, \epsilon)$ on some small polydisc $D(0, \rho) \times D\left(0, \epsilon_{0}\right)$ centered at the origin in $\mathbb{C}^{2}$ with supremum norm). We assume that all Fourier coefficients $b_{k, \beta}(x, \epsilon), c_{l, \beta}(x, \epsilon)$ have exponential decay in $\beta$ (see (177), (178)). Hence, $b_{k}(z, x, \epsilon)$ and $c_{l}(z, x, \epsilon)$ define bounded holomorphic functions on $H_{\rho^{\prime}} \times$ $\bar{D}(0, \rho) \times D\left(0, \epsilon_{0}\right)$ where $H_{\rho^{\prime}}=\left\{z \in \mathbb{C} /|\operatorname{Im}(z)|<\rho^{\prime}\right\}$ is some strip of width $2 \rho^{\prime}>0$. 
The initial data are quasiperiodic in the variable $z$ and are constructed as follows:

$$
\Xi_{i, j}(t, z, \epsilon)=\sum_{\beta_{0}, \ldots, \beta_{l} \geq 0} \Xi_{i, \beta_{0}, \ldots, \beta_{l}, j}(t, \epsilon) \frac{\exp \left(i z\left(\sum_{j=0}^{l} \beta_{j} \xi_{j}\right)\right)}{\beta_{0} ! \cdots \beta_{l} !},
$$

where $\xi_{0}=1$ and $\xi_{1}, \ldots, \xi_{l}$ are real algebraic numbers (for some integer $l \geq 1$ ) such that the family $\left\{1, \xi_{1}, \ldots, \xi_{l}\right\}$ is $\mathbb{Z}$ linearly independent and where the coefficients $\Xi_{i, \beta_{0}, \ldots, \beta_{l}, j}(t, \epsilon)$ are bounded holomorphic functions on $\mathscr{T} \times \mathscr{E}_{i}$, where $\mathscr{T}$ is a fixed open bounded sector centered at 0 and $\underline{\mathscr{E}}=\left\{\mathscr{E}_{i}\right\}_{0 \leq i \leq \nu-1}$ is a family of open bounded sectors centered at the origin and whose union forms a covering of $\mathscr{V} \backslash\{0\}$, where $\mathscr{V}$ denotes some bounded neighborhood of 0 . These functions (4) are constructed in such a way that they define holomorphic functions on $\mathscr{T} \times H_{\rho_{1}^{\prime}} \times \mathscr{E}_{i}$ for some $0<\rho_{1}^{\prime}<\rho^{\prime}$.

Recall that a function $f: \mathbb{R} \rightarrow \mathbb{E}$ (where $\mathbb{E}$ denotes some vector space) is said to be quasiperiodic with period $w=$ $\left(w_{0}, \ldots, w_{l}\right) \in \mathbb{R}^{l+1}$, for some integer $l \geq 1$, if there exists a function $F: \mathbb{R}^{l+1} \rightarrow \mathbb{E}$ such that, for all $0 \leq j \leq l$, the partial function $x_{j} \mapsto F\left(x_{0}, \ldots, x_{j}, \ldots, x_{l}\right)$ is $w_{j}$-periodic on $\mathbb{R}$, for all fixed $x_{k} \in \mathbb{R}$ when $k \neq j$, which satisfies $f(t)=F(t, \ldots, t)$ (see, e.g., [1] for a definition and properties of quasiperiodic functions). In particular, one can check that functions (4) are quasiperiodic with period $w=\left(2 \pi, 2 \pi / \xi_{1}, \ldots, 2 \pi / \xi_{l}\right)$ in the $z$ variable.

Our main purpose is the construction of actual holomorphic functions $X_{i}(t, z, x, \epsilon)$ to the problem (1), (2) on the domains $\mathscr{T} \times H_{\rho_{1}^{\prime}} \times D\left(0, \rho_{1}\right) \times \mathscr{E}_{i}$ for some small disc $D\left(0, \rho_{1}\right) \subset \mathbb{C}$ and the analysis of their asymptotic expansions as $\epsilon$ tends to zero on $\mathscr{E}_{i}$, for all $0 \leq i \leq v-1$. More precisely, we can state our main result as follows.

Main Statement. We take a set of directions $d_{i} \in \mathbb{R}, 0 \leq i \leq$ $v-1$, such that $d_{i} \neq \pi\left((2 k+1) / r_{2}\right)$, for $0 \leq k \leq r_{2}-1$, which are assumed to satisfy moreover

$$
\frac{r_{3}}{r_{2}} \arg (\epsilon)+\arg (t) \in\left(d_{i}-\theta, d_{i}+\theta\right)
$$

for all $\epsilon \in \mathscr{E}_{i}$, all $t \in \mathscr{T}$, and all $0 \leq i \leq \nu-1$, for some fixed $\theta>$ $\pi$. We make the hypothesis that the coefficients $\Xi_{i, \beta_{0}, \ldots, \beta_{l}, j}(t, \epsilon)$ of the initial data (4) can be expressed as Laplace transforms

$$
\Xi_{i, \beta_{0}, \ldots, \beta_{l}, j}(t, \epsilon)=\frac{1}{\epsilon^{r_{3} / r_{2} t}} \int_{L_{d_{i}}} V_{i, \beta_{0}, \ldots, \beta_{l}, j}(\tau, \epsilon) e^{-\tau /\left(\epsilon^{r_{3} / r_{2}} t\right)} d \tau
$$

on $\mathscr{T} \times \mathscr{E}_{i}$ along the half-line $L_{d_{i}}=\mathbb{R}_{+} e^{\sqrt{-1} d_{i}}$, where $V_{i, \beta_{0}, \ldots, \beta_{l}, j}(\tau, \epsilon)$ is a family of holomorphic functions which share the exponential growth constraints (152) with respect to $\tau$, the uniform bound estimates (161), and the analytic continuation property (184).

Then, in Proposition 28, we construct a family of holomorphic and bounded functions

$$
X_{i}(t, z, x, \epsilon)=\sum_{\beta_{0}, \ldots, \beta_{l} \geq 0} X_{i, \beta_{0}, \ldots, \beta_{l}}(t, x, \epsilon) \frac{\exp \left(i z\left(\sum_{j=0}^{l} \beta_{j} \xi_{j}\right)\right)}{\beta_{0} ! \cdots \beta_{l} !}
$$

which are quasiperiodic with period $w=\left(2 \pi, 2 \pi / \xi_{1}, \ldots\right.$, $\left.2 \pi / \xi_{l}\right)$ in the variable $z$ and which solve the problem (1), (2) on the products $\mathscr{T} \times H_{\rho_{1}^{\prime}} \times D\left(0, \rho_{1}\right) \times \mathscr{E}_{i}$, where $\rho_{1}^{\prime}>$ 0 satisfies inequality (153) and for some small radius $0<$ $\rho_{1}<\rho$. Moreover, the differences $X_{i+1}(t, z, x, \epsilon)-X_{i}(t, z, x, \epsilon)$ satisfy the exponential decay (187) whose type depends on the constants $r_{1}, r_{2}, r_{3}$ and on the degree $h+1$ of any algebraic number field $\mathbb{Q}(\xi)$ containing $\xi_{1}, \ldots, \xi_{l}$.

In Theorem 32, we show the existence of a formal series

$$
\widehat{X}(\epsilon)=\sum_{k \geq 0} H_{k}(t, z, x) \frac{\epsilon^{k}}{k !}
$$

whose coefficients $H_{k}(t, z, x)$ belong to the Banach space of bounded holomorphic functions on $\mathscr{T} \times H_{\rho_{1}^{\prime}} \times D\left(0, \rho_{1}\right)$, which formally solves (1) and is moreover the Gevrey asymptotic expansion of order $\left(h r_{1}+r_{2}\right) / r_{3}$ of $X_{i}$ on $\mathscr{E}_{i}$. In other words, there exist two constants $C, M>0$ such that

$$
\begin{aligned}
& \sup _{t \in \mathscr{T}, z \in H_{\rho_{1}^{\prime}}, x \in D\left(0, \rho_{1}\right)}\left|X_{i}(t, z, x, \epsilon)-\sum_{k=0}^{N-1} H_{k}(t, z, x) \frac{\epsilon^{k}}{k !}\right| \\
& \leq C M^{N} N !^{\left(h r_{1}+r_{2}\right) / r_{3}}|\epsilon|^{N}
\end{aligned}
$$

for all $N \geq 1$ and all $\epsilon \in \mathscr{E}_{i}$.

Notice that the problem (1), (2) is singularly perturbed with irregular singularity (in the sense of Mandai, [2]) with respect to $t$ at $t=0$ provided that $r_{2}>r_{1}$. It is of Kowalevski type if $r_{2}<r_{1}$ (meaning that the hypotheses of the classical Cauchy-Kowalevski theorem (see, e.g., [3], pp. 346-349) are fulfilled for (1)) and of mixed type when $r_{2}$ and $r_{1}$ are equal.

In a recent work [4], we have considered singularly perturbed nonlinear Cauchy problems of the form

$$
\begin{aligned}
& \epsilon^{r_{3}}\left(z \partial_{z}\right)^{r_{1}}\left(t^{2} \partial_{t}\right)^{r_{2}} \partial_{z}^{S} u_{i}(t, z, \epsilon) \\
& \quad=F\left(t, z, \epsilon, \partial_{t}, \partial_{z}\right) u_{i}(t, z, \epsilon)+P\left(t, z, \epsilon, u_{i}(t, z, \epsilon)\right)
\end{aligned}
$$

which carry both an irregular singularity with respect to $t$ at $t=0$ and a Fuchsian singularity (see [5] for a definition) with respect to $z$ at $z=0$, for given initial data

$$
\begin{aligned}
& \left(\partial_{z}^{j} u_{i}\right)(t, 0, \epsilon)=\phi_{i, j}(t, \epsilon), \\
& 0 \leq i \leq v-1, \quad 0 \leq j \leq S-1,
\end{aligned}
$$

where $F$ is some linear differential operator with polynomial coefficients and $P$ is some polynomial. The initial data $\phi_{j, i}(t, \epsilon)$ were assumed to be holomorphic on products $\mathscr{T} \times \mathscr{E}_{i}$. Under suitable constraints on the shape of (10) and on the initial data (11), we have shown the existence of a formal series $\widehat{u}(\epsilon)=\sum_{k \geq 0} h_{k} \epsilon^{k} / k$ ! with coefficients $h_{k}$ belonging to the Banach space $\mathbb{F}$ of bounded holomorphic functions on $\mathscr{T} \times$ $D(0, \delta)$ (for some $\delta>0$ ) equipped with the supremum norm, solution of (10), which is the Gevrey asymptotic expansion of order $\left(r_{1}+r_{2}\right) / r_{3}$ of actual holomorphic solutions $u_{i}$ of (10), (11) on $\mathscr{E}_{i}$ as $\mathbb{F}$-valued functions, for all $0 \leq i \leq v-1$.

Compared to this former result [4], the singularity nature of (1) does not come from the divergence of the formal 
series. This divergence rather emerges from the quasiperiodic structure of the solution space which produces a small divisor problem (as we will see below) and its Gevrey type depends not only on the type of space of our initial data but also on the shape of (1). It is worth noticing that a similar phenomenon has been observed in the paper [6] for the steady SwiftHohenberg equation

$$
(1+\Delta)^{2} U(\mathbf{x}, \mu)-\mu U(\mathbf{x}, \mu)+U^{3}(\mathbf{x}, \mu)=0
$$

where the authors have constructed formal series solutions

$$
U(\mathbf{x}, \mu)=\sqrt{\mu} \sum_{n \geq 0} U^{(n)}(\mathbf{x}) \mu^{n},
$$

where the coefficients $U^{(n)}(\mathbf{x})$ belong to some weighted Sobolev space $H^{s}(\Gamma)$ (for well-chosen real number $s>0$ ) of quasiperiodic Fourier expansions in $\mathbf{x} \in \mathbb{R}^{2}$ of the form

$$
U^{(n)}(\mathbf{x})=\sum_{\mathbf{k} \in \Gamma} U_{\mathbf{k}} e^{i \mathbf{k} \cdot \mathbf{x}},
$$

where $\Gamma=\left\{\sum_{j=1}^{Q} m_{j} \mathbf{k}_{j} /\left(m_{1}, \ldots, m_{Q}\right) \in \mathbb{N}^{Q}\right\}$ with $\mathbf{k}_{j}=$ $(\cos (2 \pi((j-1) / Q)), \sin (2 \pi((j-1) / Q)))$ is the so-called quasilattice in $\mathbb{R}^{2}$ for some integer $Q \geq 8$. They have shown that this formal series (13) is actually at most of Gevrey order $4 l$ (for a suitable integer $l$ depending on $Q$ ) as series in the Hilbert space $H^{s}(\Gamma)$. Their main purpose was actually to use this result in order to construct approximate smooth quasiperiodic solutions of (12) up to an exponential small order by means of truncated Laplace transforms.

In a more general setting, the Cauchy problem (1), (2) we consider in this work comes within the framework of asymptotic analysis of solutions to differential equations or to partial differential equations with periodic or quasiperiodic coefficients which is a domain of intense research in these last years.

In the category of differential equations most of the results concern nonlinear equations of the form

$$
\sum_{k=0}^{K} a_{k}(\epsilon) \partial_{t}^{k} u(t, \epsilon)=F(u(t, \epsilon), t, \epsilon),
$$

where the forcing term $F$ contains periodic or quasiperiodic coefficients. These statements deal with the construction of formal solutions $u(t, \epsilon)=\sum_{l=0}^{+\infty} u_{l}(t) \epsilon^{l}$ which are called Lindstedt series in the literature. For convergence properties of these series, we quote the seminal work [7] and the overview [8], and for Borel resummation procedures applied more recently, we mention [9]. For applications in KAM theory for nearly integrable finitely dimensional Hamiltonian systems, we may refer to $[10,11]$.

In the context of partial differential equations, for existence results of quasiperiodic solutions to general families of nonlinear PDE containing a small real parameter, we indicate [12] and for the construction of periodic solutions to abstract second order nonlinear equations, we notice [13]. Concerning KAM theory results in the context of PDE such as small nonlinear perturbations of wave equations or Schrödinger equations we mention the fundamental works [14-16].
Now, we explain our main result and the principal arguments needed in its proof. The first step consists (as in [4]) of transforming (1) by means of the linear map $T \mapsto T / \epsilon^{r_{3} / r_{2}}$ into an auxiliary regularly perturbed nonlinear equation (149). The drawback of this transformation is the appearance of poles in the coefficients of this new equation with respect to $\epsilon$ at 0 .

The approach we follow is the same as in our previous works $[4,17]$ and is based on a Borel resummation procedure applied to formal expansions of the form

$$
\begin{aligned}
& \widehat{Y}(T, z, x, \epsilon) \\
& =\sum_{\underline{\beta}=\left(\beta_{0}, \ldots, \beta_{l}, \beta_{l+1}\right) \in \mathbb{N}^{l+2}} \widehat{Y}_{\beta}(T, \epsilon) \frac{\exp \left(i z\left(\sum_{j=0}^{l} \beta_{j} \xi_{j}\right)\right)}{\beta_{0} ! \cdots \beta_{l} !} \frac{x^{\beta_{l+1}}}{\beta_{l+1} !},
\end{aligned}
$$

where $\widehat{Y}_{\underline{\beta}}(T, \epsilon)=\sum_{m \geq 0} \chi_{m, \underline{\beta}}(\epsilon) T^{m} / m$ ! are formal series in $T$, which formally solves the auxiliary equation (149) for well-chosen initial data (165). It is worth pointing out that this resummation method known as $\kappa$-summability already enjoys a large success in the study of Gevrey asymptotics for analytic solutions to linear and nonlinear differential equations with irregular singularity; see, for instance, [18-24]. We show that the formal Borel transform of $\widehat{Y}(T, z, x, \epsilon)$ with respect to $T$ given by

$$
\begin{aligned}
& \widehat{V}(\tau, z, x, \epsilon) \\
& =\sum_{\underline{\beta}=\left(\beta_{0}, \ldots, \beta_{l}, \beta_{l+1}\right) \in \mathbb{N}^{l+2}} \widehat{V}_{\beta}(\tau, \epsilon) \frac{\exp \left(i z\left(\sum_{j=0}^{l} \beta_{j} \xi_{j}\right)\right)}{\beta_{0} ! \cdots \beta_{l} !} \frac{x^{\beta_{l+1}}}{\beta_{l+1} !},
\end{aligned}
$$

where $\widehat{V}_{\beta}(\tau, \epsilon)=\sum_{m \geq 0} \chi_{m, \beta}(\epsilon) \tau^{m} /(m !)^{2}$, formally solves a nonlinear convolution integrodifferential Cauchy problem with rational coefficients in $\tau$ and is holomorphic with respect to $x$ near the origin and with respect to $z$ in some strip and meromorphic in $\epsilon$ with a pole at 0 ; see (171), (172).

For appropriate initial data satisfying conditions (152), (184), and (161), we show (in Proposition 20) that the formal series $\widehat{V}(\tau, z, x, \epsilon)$ actually defines a holomorphic function $V_{i}$ on the product $U_{i} \times H_{\rho_{1}^{\prime}} \times D\left(0, \rho_{1}\right) \times D\left(0, \epsilon_{0}\right) \backslash\{0\}$, for some $0<\rho_{1}^{\prime}<\rho^{\prime}, 0<\rho_{1}<\rho$ and where $U_{i}$ is some unbounded open sector with small aperture and with bisecting direction $d_{i}$ (as described above in the main statement). The functions $V_{i}$ have exponential growth rate with respect to $(\tau, \epsilon)$ meaning that there exist two constants $C, K>0$ such that

$$
\sup _{z \in H_{\rho_{1}^{\prime}}, x \in D\left(0, \rho_{1}\right)}\left|V_{i}(\tau, z, x, \epsilon)\right| \leq C e^{K|\tau| /|\epsilon|}
$$

for all $\tau \in U_{i}, \epsilon \in D\left(0, \epsilon_{0}\right) \backslash\{0\}$. Moreover, we show that, for all $\underline{\beta}=\left(\beta_{0}, \ldots, \beta_{l}, \beta_{l+1}\right) \in \mathbb{N}^{l+2}$, the formal series $\widehat{V}_{\underline{\beta}}(\tau, \epsilon)$ actually define holomorphic functions $V_{i, \beta}(\tau, \epsilon)$ on domains $\left(U_{i} \cup D\left(0, \rho_{\beta}\right)\right) \times D\left(0, \epsilon_{0}\right) \backslash\{0\}$, where $\rho_{\underline{\beta}}$ is a Riemann type sequence of the form $R /(1+|\beta|)^{h r_{1} / r_{2}}$, for some constant 
$R>0$, which tends to 0 as $|\underline{\beta}|=\sum_{j=0}^{l+1} \beta_{j}$ tends to infinity, and share the same exponential growth rate, namely, that there exist constants $C>0, K>0, M>0$ with

$$
\sup _{z \in H_{\rho_{1}^{\prime}}, x \in D\left(0, \rho_{1}\right)}\left|V_{i, \underline{\beta}}(\tau, \epsilon)\right| \leq C K^{\sum_{j=0}^{l+1} \beta_{j}} \beta_{0} ! \cdots \beta_{l+1} ! e^{M|\tau| /|\epsilon|}
$$

for all $\tau \in U_{i}, \epsilon \in D\left(0, \epsilon_{0}\right) \backslash\{0\}$. We point out that the occurrence of a radius of convergence shrinking to zero for the coefficients $V_{i, \beta}$ near the origin of the Borel transform is due to the presence of a small divisor phenomenon in the convolution Cauchy problem (171), (172) mentioned above. In our previous study [4], a similar outcome was caused by a leading term in the main equation (10) containing a Fuchsian operator $\left(z \partial_{z}\right)^{r_{1}}$. In this analysis, the denominators arise from the function space where the solutions are found, especially from their Fourier exponents $\sum_{j=0}^{l+1} \beta_{j} \xi_{j}$ which may tend to zero but not faster than a Riemann type sequence as follows from Lemma 13.

In order to get the estimates described above, we use a majorizing technique described in Propositions 17, 18, and 19 which reduces the investigation for bounds (19) to the study of a Cauchy-Kowalevski type problem (114), (115) in several complex variables for which local analytic solutions are found in Section 2.1; see Proposition 5. On the way we make use of estimates in weighted Banach spaces introduced in Section 2.2; see Propositions 9, 10, and 12 and Corollary 11, which are very much like those already seen in the work [4].

In the next step, for given suitable initial data (150) satisfying (158), we construct actual solutions

$$
\begin{aligned}
& Y_{i}(T, z, x, \epsilon) \\
& =\sum_{\underline{\beta}=\left(\beta_{0}, \ldots, \beta_{l}, \beta_{l+1}\right) \in \mathbb{N}^{l+2}} Y_{i, \underline{\beta}}(T, \epsilon) \frac{\exp \left(i z\left(\sum_{j=0}^{l} \beta_{j} \xi_{j}\right)\right)}{\beta_{0} ! \cdots \beta_{l} !} \frac{x^{\beta_{l+1}}}{\beta_{l+1} !}
\end{aligned}
$$

of (149), where each function $Y_{i, \beta}(T, \epsilon)$ can be written as a Laplace transform of the function $V_{i, \beta}(\tau, \epsilon)$ with respect to $\tau$ along a half-line $L_{\gamma_{i}}=\mathbb{R}_{+} e^{i \sqrt{-1} \gamma_{i}} \subset U_{i} \cup\{0\}$. For each $\epsilon \in \mathscr{E}_{i}$, the function $T \mapsto Y_{i, \beta}(T, \epsilon)$ is bounded and holomorphic on a sector $U_{i, \epsilon}$ with aperture larger than $\pi$, with bisecting direction $\gamma_{i}$ and with radius $h^{\prime}|\epsilon|^{r_{3} / r_{2}}$ for some constant $h^{\prime}>0$. In Proposition 23, we show that the function $Y_{i}$ itself turns out to define a holomorphic function on $U_{i, \epsilon} \times H_{\rho_{1}^{\prime}} \times D\left(0, \rho_{1}\right)$ for some $0<\rho_{1}<\rho$ and where $\rho_{1}^{\prime}>0$ satisfies (153).

We observe that, for all $0 \leq i \leq v-1$, the functions $X_{i}$ defined as

$$
X_{i}(t, z, x, \epsilon)=Y_{i}\left(\epsilon^{r_{3} / r_{2}} t, z, x, \epsilon\right)
$$

actually solve our initial Cauchy problem (1), (2) on the products $\mathscr{T} \times H_{\rho_{1}^{\prime}} \times D\left(0, \rho_{1}\right) \times \mathscr{E}_{i}$ and bear representation (7) as a quasiperiodic function whose Fourier coefficients decay exponentially in $\beta$. It is worthy to mention that spaces of quasiperiodic Fourier series with exponential decay were also recently used in [25] in order to find global in time and quasiperiodic in space solutions to the KdV equation.

In Proposition 28, we show moreover that the difference of two neighboring solutions $X_{i+1}$ and $X_{i}$ has exponentially small bounds of order $r_{3} /\left(h r_{1}+r_{2}\right)$, uniformly in $(t, z, x)$, as $\epsilon$ tends to 0 on $\mathscr{E}_{i+1} \cap \mathscr{E}_{i}$. We observe that for each $\underline{\beta} \in \mathbb{N}^{l+2}$ the difference $X_{i+1, \beta}-X_{i, \beta}$ for the Fourier coefficients has exponential decay of order $r_{3} / r_{2}$ but its type is proportional to $\rho_{\beta}$ and therefore tends to 0 as $\beta$ tends to infinity. This small denominator phenomenon is the reason for the decreasement of the order $r_{3} / r_{2}$ to $r_{3} /\left(h r_{1}+r_{2}\right)$. As in our previous study [4], the bulk of the proof rests on a thorough estimation of a Dirichlet like series of the form $\sum_{k \geq 0} e^{-1 /(k+1)^{\alpha} \epsilon^{r}} a^{k}$ for $0<a<1$ and $\alpha, r>0$ with $\epsilon>0$ small. This kind of series appears in the context of almost periodic functions introduced by $H$. Bohr; see, for instance, the textbook [26]. These estimates (187) are crucial in order to apply a cohomological criterion known in the literature as the Ramis-Sibuya theorem (Theorem RS) which leads to the main result of this paper, namely, the existence of a formal series

$$
\widehat{X}(t, z, x, \epsilon)=\sum_{k \geq 0} H_{k} \frac{\epsilon^{k}}{k !}
$$

with coefficients $H_{k}$ in the Banach space of holomorphic and bounded functions on $\mathscr{T} \times H_{\rho_{1}^{\prime}} \times D\left(0, \rho_{1}\right)$, which formally solves (1) and which is, moreover, the Gevrey asymptotic expansion of order $\left(h r_{1}+r_{2}\right) / r_{3}$ of the functions $X_{i}$ on $\mathscr{E}_{i}$, for all $0 \leq i \leq v-1$.

The layout of the paper reads as follows.

Section 2.1 is dedicated to the study of a version of the Cauchy-Kowalevski theorem for nonlinear PDEs in analytic spaces of functions with precise control on the domain of existence of their solutions in terms of norm estimates of the initial data. In Section 2.2 we establish some continuity properties of several integrodifferential and multiplication operators acting on weighted Banach spaces of holomorphic functions. These results are applied in Section 2.3 when looking for global solutions with growth constraint at infinity for a parameter depending nonlinear convolution differential Cauchy problem with singular coefficients.

We recall briefly the classical theory concerning the Borel-Laplace transform and we show some commutation formulas with multiplication and integrodifferential operators in Section 3.1; then we center our attention on finding solutions of an auxiliary nonlinear Cauchy problem obtained by the linear change of variable $T \mapsto T / \epsilon^{r}$ from our main Cauchy problem in Section 3.2. The link between this Cauchy problem and the one solved in Section 2.3 is performed by means of Borel-Laplace transforms on the corresponding solutions.

In Section 4.1, we construct actual holomorphic solutions $X_{i}, 0 \leq i \leq v-1$, of our initial problem and we show exponential decay of the difference of any two of these solutions with respect to $\epsilon$ on the intersection of their domain of definition, uniformly in the other variables. Finally, in Section 4.2, we conclude with the main result of the work, 
that is, the existence of a formal power series with coefficients in an appropriate Banach space, which asymptotically represents the functions $X_{i}$ with a precise control on the Gevrey order on the sectors $\mathscr{E}_{i}$, for all $0 \leq i \leq v-1$.

\section{A Global Cauchy Problem in Holomorphic and Quasiperiodic Function Spaces}

2.1. A Cauchy-Kowalevski Theorem in Several Variables. In this section, we recall the well-known Cauchy-Kowalevski theorem in some spaces of analytic functions for which the size of the domain of existence of the solution can be controlled in terms of some supremum norm of the initial data.

The next Banach spaces are natural extensions to the several variables cases of the spaces used in [27].

Definition 1. Let $l \geq 1$ be some integer. Let $\bar{Z}_{0}, \ldots, \bar{Z}_{l}, \bar{X}>0$ be positive real numbers. We denote by $G\left(\bar{Z}_{0}, \ldots, \bar{Z}_{l}, \bar{X}\right)$ the space of formal series

$$
U\left(Z_{0}, \ldots, Z_{l}, X\right)=\sum_{\underline{\beta}=\left(\beta_{0}, \ldots, \beta_{l}, \beta_{l+1}\right) \in \mathbb{N}^{l+2}} u_{\underline{\beta}} \frac{Z_{0}^{\beta_{0}} \cdots Z_{l}^{\beta_{l}} X^{\beta_{l+1}}}{\beta_{0} ! \cdots \beta_{l} ! \beta_{l+1} !}
$$

that belong to $\mathbb{C}\left[\left[Z_{0}, \ldots, Z_{l}, X\right]\right]$ such that

$$
\begin{aligned}
& \left\|U\left(Z_{0}, \ldots, Z_{l}, X\right)\right\|_{\left(\bar{Z}_{0}, \ldots, \bar{Z}_{l}, \bar{X}\right)} \\
& =\sum_{\underline{\beta}=\left(\beta_{0}, \ldots, \beta_{l}, \beta_{l+1}\right) \in \mathbb{N}^{l+2}}\left|u_{\underline{\beta}}\right| \frac{\bar{Z}_{0}^{\beta_{0}} \cdots \bar{Z}_{l}^{\beta_{l}} \bar{X}^{\beta_{l+1}}}{\left(\sum_{j=0}^{l+1} \beta_{j}\right) !}
\end{aligned}
$$

is finite. One can show that $G\left(\bar{Z}_{0}, \ldots, \bar{Z}_{l}, \bar{X}\right)$ equipped with the norm $\|\cdot\|_{\left(\bar{Z}_{0}, \ldots, \bar{Z}_{l}, \bar{X}\right)}$ are Banach spaces.

In the next two lemmas we show continuity properties for some linear integrodifferential operators acting on the aforementioned Banach spaces.

Lemma 2. Let $h_{0}, \ldots, h_{l}, h_{l+1} \in \mathbb{N}$ with

$$
h_{l+1} \geq \sum_{j=0}^{l} h_{j}
$$

Then, for any given $\bar{Z}_{0}, \ldots, \bar{Z}_{l}, \bar{X}>0$, the operator $\partial_{Z_{0}}^{h_{0}} \ldots$ $\partial_{Z_{l}}^{h_{l}} \partial_{X}^{-h_{l+1}}$ is a bounded linear map from $G\left(\bar{Z}_{0}, \ldots, \bar{Z}_{l}, \bar{X}\right)$ into itself. Moreover,

$$
\begin{aligned}
\| \partial_{Z_{0}}^{h_{0}} & \cdots \partial_{Z_{l}}^{h_{l}} \partial_{X}^{-h_{l+1}} U\left(Z_{0}, \ldots, Z_{l}, X\right) \|_{\left(\bar{Z}_{0}, \ldots, \bar{Z}_{l}, \bar{X}\right)} \\
& \leq \bar{Z}_{0}^{h_{0}} \ldots \bar{Z}_{l}{ }_{l}^{h_{l}} \bar{X}^{h_{l+1}}\left\|U\left(Z_{0}, \ldots, Z_{l}, X\right)\right\|_{\left(\bar{Z}_{0}, \ldots, \bar{Z}_{l}, \bar{X}\right)}
\end{aligned}
$$

for all $U\left(Z_{0}, \ldots, Z_{l}, X\right) \in G\left(\bar{Z}_{0}, \ldots, \bar{Z}_{l}, \bar{X}\right)$.
Proof. Let $U\left(Z_{0}, \ldots, Z_{l}, X\right) \in G\left(\bar{Z}_{0}, \ldots, \bar{Z}_{l}, \bar{X}\right)$ of form (23). By definition, we can write

$$
\begin{gathered}
\left\|\partial_{Z_{0}}^{h_{0}} \cdots \partial_{Z_{l}}^{h_{l}} \partial_{X}^{-h_{l+1}} U\left(Z_{0}, \ldots, Z_{l}, X\right)\right\|_{\left(\bar{Z}_{0}, \ldots, \overline{Z_{l}}, \bar{X}\right)} \\
=\sum_{\beta_{0}, \ldots, \beta_{l}, \beta_{l+1} \geq 0}\left(\frac{\left(\sum_{j=0}^{l} \beta_{j}+h_{j}+\beta_{l+1}-h_{l+1}\right) !}{\left(\sum_{j=0}^{l+1} \beta_{j}\right) !}\right. \\
\left.\cdot \bar{Z}_{0}^{-h_{0}} \cdots \bar{Z}_{l}^{-h_{l}} \bar{X}^{h_{l+1}}\right) \\
\cdot \frac{\mid U_{\beta_{0}+h_{0}, \ldots, \beta_{l}+h_{l}, \beta_{l+1}-h_{l+1} \mid}}{\left(\sum_{j=0}^{l} \beta_{j}+h_{j}+\beta_{l+1}-h_{l+1}\right) !} \\
\cdot \bar{Z}_{0}^{\beta_{0}+h_{0}} \ldots \bar{Z}_{l}^{\beta_{l}+h_{l}} \bar{X}^{\beta_{l+1}-h_{l+1}} .
\end{gathered}
$$

From (25), we have

$$
\frac{\left(\sum_{j=0}^{l} \beta_{j}+h_{j}+\beta_{l+1}-h_{l+1}\right) !}{\left(\sum_{j=0}^{l+1} \beta_{j}\right) !} \leq 1
$$

for all $\beta_{0}, h_{0}, \ldots, \beta_{l}, h_{l}, \beta_{l+1}, h_{l+1} \geq 0$. Estimate (26) follows.

Lemma 3. Let $h_{0}, \ldots, h_{l}, h_{l+1} \in \mathbb{N}$. Let, for all $0 \leq j \leq l, 0<$ $\bar{Z}_{j}^{1}<\bar{Z}_{j}^{0}$ and $0<\bar{X}^{1}<\bar{X}^{0}$ be positive real numbers. Then, there exists $C_{2}>0$ (depending on $h_{0}, \ldots, h_{l+1}, \bar{X}^{0}, \bar{X}^{1}, \bar{Z}_{j}^{0}, \bar{Z}_{j}^{1}$ for $0 \leq j \leq l$ ) such that

$$
\begin{gathered}
\left\|\partial_{Z_{0}}^{h_{0}} \ldots \partial_{Z_{l}}^{h_{l}} \partial_{X}^{h_{l+1}} U\left(Z_{0}, \ldots, Z_{l}, X\right)\right\|_{\left(\bar{Z}_{0}^{1}, \ldots, \bar{Z}_{l}^{1}, \bar{X}^{1}\right)} \\
\leq C_{2}\left(\bar{Z}_{0}^{1}\right)^{-h_{0}} \ldots\left(\bar{Z}_{l}^{1}\right)^{-h_{l}}\left(\bar{X}^{1}\right)^{-h_{l+1}} \\
\cdot\left\|U\left(Z_{0}, \ldots, Z_{l}, X\right)\right\|_{\left(\bar{Z}_{0}^{0}, \ldots, \bar{Z}_{l}^{0}, \bar{X}^{0}\right)}
\end{gathered}
$$

for all $U\left(Z_{0}, \ldots, Z_{l}, X\right) \in G\left(\bar{Z}_{0}^{0}, \ldots, \bar{Z}_{l}^{0}, \bar{X}^{0}\right)$.

Proof. Let $U\left(Z_{0}, \ldots, Z_{l}, X\right) \in G\left(\bar{Z}_{0}^{0}, \ldots, \bar{Z}_{l}^{0}, \bar{X}^{0}\right)$ be of form (23). By definition, we can write

$$
\begin{gathered}
\left\|\partial_{Z_{0}}^{h_{0}} \cdots \partial_{Z_{l}}^{h_{l}} \partial_{X}^{h_{l+1}} U\left(Z_{0}, \ldots, Z_{l}, X\right)\right\|_{\left(\bar{Z}_{0}^{1}, \ldots, \bar{Z}_{l}^{1}, \bar{X}^{1}\right)} \\
=\sum_{\beta_{0}, \ldots, \beta_{l}, \beta_{l+1} \geq 0} \frac{\left|u_{\beta_{0}+h_{0}, \ldots, \beta_{l}+h_{l}, \beta_{l+1}+h_{l+1} \mid}\right|}{\left(\sum_{j=0}^{l+1} \beta_{j}+h_{j}\right) !} \\
\cdot\left(\bar{Z}_{0}^{1}\right)^{-h_{0}} \cdots\left(\bar{Z}_{l}^{1}\right)^{-h_{l}}\left(\bar{X}^{1}\right)^{-h_{l+1}} \\
\cdot\left(\frac{\left(\sum_{j=0}^{l+1} \beta_{j}+h_{j}\right) !}{\left(\sum_{j=0}^{l+1} \beta_{j}\right) !} \times\left(\bar{Z}_{0}^{1}\right)^{\beta_{0}+h_{0}} \cdots\left(\bar{Z}_{l}^{1}\right)^{\beta_{l}+h_{l}}\right. \\
\left.\cdot\left(\bar{X}^{1}\right)^{\beta_{l+1}+h_{l+1}}\right) .
\end{gathered}
$$


Now, we take some real number $0<\delta<1$ such that $\bar{Z}_{j}^{1}<\delta \bar{Z}_{j}^{0}$ for all $0 \leq j \leq l$ and $\bar{X}^{1}<\delta \bar{X}^{0}$. We get the estimates

$$
\begin{gathered}
\frac{\left(\sum_{j=0}^{l+1} \beta_{j}+h_{j}\right) !}{\left(\sum_{j=0}^{l+1} \beta_{j}\right) !}\left(\bar{Z}_{0}^{1}\right)^{\beta_{0}+h_{0}} \cdots\left(\bar{Z}_{l}^{1}\right)^{\beta_{l}+h_{l}}\left(\bar{X}^{1}\right)^{\beta_{l+1}+h_{l+1}} \\
\leq\left(\sum_{j=0}^{l+1} \beta_{j}+\sum_{j=0}^{l+1} h_{j}\right)^{\left(\sum_{j=0}^{l+1} h_{j}\right)} \delta^{\left(\sum_{j=0}^{l+1} \beta_{j}+\sum_{j=0}^{l+1} h_{j}\right)} \\
\cdot\left(\bar{Z}_{0}^{0}\right)^{\beta_{0}+h_{0}} \cdots\left(\bar{Z}_{l}^{0}\right)^{\beta_{l}+h_{l}}\left(\bar{X}^{0}\right)^{\beta_{l+1}+h_{l+1}}
\end{gathered}
$$

for all $\beta_{0}, h_{0}, \ldots, \beta_{l}, h_{l}, \beta_{l+1}, h_{l+1} \geq 0$. Since $0<\delta<1$, we know that, for any real number $a>0$, there exists $K_{\delta, a}>0$ depending on $a, \delta$ such that

$$
\sup _{n \geq 0}(n+a)^{a} \delta^{n+a} \leq K_{\delta, a}
$$

Hence, we get a constant $C_{2}>0$ depending on $h_{j}, 0 \leq j \leq l+1$ and $\delta$ with

$$
\begin{gathered}
\left(\sum_{j=0}^{l+1} \beta_{j}+\sum_{j=0}^{l+1} h_{j}\right)^{\left(\sum_{j=0}^{l+1} h_{j}\right)} \delta^{\left(\sum_{j=0}^{l+1} \beta_{j}+\sum_{j=0}^{l+1} h_{j}\right)} \\
\cdot\left(\bar{Z}_{0}^{0}\right)^{\beta_{0}+h_{0}} \cdots\left(\bar{Z}_{l}^{0}\right)^{\beta_{l}+h_{l}}\left(\bar{X}^{0}\right)^{\beta_{l+1}+h_{l+1}} \\
\leq C_{2}\left(\bar{Z}_{0}^{0}\right)^{\beta_{0}+h_{0}} \cdots\left(\bar{Z}_{l}^{0}\right)^{\beta_{l}+h_{l}}\left(\bar{X}^{0}\right)^{\beta_{l+1}+h_{l+1}}
\end{gathered}
$$

for all $\beta_{0}, h_{0}, \ldots, \beta_{l}, h_{l}, \beta_{l+1}, h_{l+1} \geq 0$. As a result, gathering (30), (31), and (33), we get the lemma.

Lemma 4. Let $U_{1}\left(Z_{0}, \ldots, Z_{l}, X\right), U_{2}\left(Z_{0}, \ldots, Z_{l}, X\right) \in G\left(\bar{Z}_{0}\right.$, $\left.\ldots, \bar{Z}_{l}, \bar{X}\right)$. Then, the product $U_{1} U_{2}$ belongs to $G\left(\bar{Z}_{0}, \ldots\right.$, $\left.\bar{Z}_{l}, \bar{X}\right)$. Moreover, we have

$$
\begin{gathered}
\left\|U_{1}\left(Z_{0}, \ldots, Z_{l}, X\right) U_{2}\left(Z_{0}, \ldots, Z_{l}, X\right)\right\|_{\left(\bar{Z}_{0}, \ldots, \bar{Z}_{l}, \bar{X}\right)} \\
\leq\left\|U_{1}\left(Z_{0}, \ldots, Z_{l}, X\right)\right\|_{\left(\bar{Z}_{0}, \ldots, \bar{Z}_{l}, \bar{X}\right)} \\
\cdot\left\|U_{2}\left(Z_{0}, \ldots, Z_{l}, X\right)\right\|_{\left(\bar{Z}_{0}, \ldots, \bar{Z}_{l}, \bar{X}\right)} \cdot
\end{gathered}
$$

In other words, the space $G\left(\bar{Z}_{0}, \ldots, \bar{Z}_{l}, \bar{X}\right)$ is a Banach algebra. Proof. Let

$$
U_{j}\left(Z_{0}, \ldots, Z_{l}, X\right)=\sum_{\beta_{0}, \ldots, \beta_{l}, \beta_{l+1} \geq 0} u_{\beta}^{j} \frac{Z_{0}^{\beta_{0}} \cdots Z_{l}^{\beta_{l}} X^{\beta_{l+1}}}{\beta_{0} ! \cdots \beta_{l} ! \beta_{l+1} !}
$$

belonging to $G\left(\bar{Z}_{0}, \ldots, \bar{Z}_{l}, \bar{X}\right)$ for $j=1,2$. By definition, we can write

$$
\begin{gathered}
\left\|U_{1}\left(Z_{0}, \ldots, Z_{l}, X\right) U_{2}\left(Z_{0}, \ldots, Z_{l}, X\right)\right\|_{\left(\bar{Z}_{0}, \ldots, \bar{Z}_{l}, \bar{X}\right)} \\
\leq \sum_{\beta_{0}, \ldots, \beta_{l}, \beta_{l+1} \geq 0}\left(\sum_{\beta_{j}^{1}+\beta_{j}^{2}=\beta_{j}, 0 \leq j \leq l+1}\left(\frac{\prod_{j=0}^{l+1} \beta_{j} !}{\prod_{j=0}^{l+1} \beta_{j}^{1} \Pi_{j=0}^{l+1} \beta_{j}^{2} !} \frac{1}{\left(\sum_{j=0}^{l+1} \beta_{j}\right) !}\right)\right. \\
\left.\cdot \mid u_{\left(\beta_{0}^{1}, \ldots, \beta_{l}^{1}, \beta_{l+1}^{1}\right)}^{1} u_{\left(\beta_{0}^{2}, \ldots, \beta_{l}^{2}, \beta_{l+1}^{2}\right)}^{2}\right) \\
\cdot \bar{Z}_{0}^{\beta_{0}} \ldots \bar{Z}_{l}^{\beta_{l}} \bar{X}^{\beta_{l+1}} .
\end{gathered}
$$

Besides, using the identity $(1+x)^{\sum_{j=0}^{l+1} \beta_{j}}=\Pi_{j=0}^{l+1}(1+x)^{\beta_{j}}$ and the binomial formula, we get that

$$
\frac{\Pi_{j=0}^{l+1} \beta_{j} !}{\prod_{j=0}^{l+1} \beta_{j}^{1} ! \Pi_{j=0}^{l+1} \beta_{j}^{2} !} \leq \frac{\left(\sum_{j=0}^{l+1} \beta_{j}\right) !}{\left(\sum_{j=0}^{l+1} \beta_{j}^{1}\right) !\left(\sum_{j=0}^{l+1} \beta_{j}^{2}\right) !}
$$

for all integers $\beta_{j}, \beta_{j}^{1}, \beta_{j}^{2} \geq 0$ such that $\beta_{j}=\beta_{j}^{1}+\beta_{j}^{2}$, for $0 \leq$ $j \leq l+1$. Therefore, inequality (34) follows from (36) and (37).

In the next proposition, we state a version of the CauchyKowalevski theorem.

Proposition 5. Let $\mathscr{D}_{1}\left(\right.$ resp., $\left.\mathscr{D}_{2}\right)$ be a finite subset of $\mathbb{N}^{l+2}$ (resp., $\mathbb{N}^{l+1}$ ) and let $S \geq 1$ be an integer such that, for all $\left(k_{0}, \ldots, k_{l+1}\right) \in \mathscr{D}_{1}$ and all $\left(p_{0}, \ldots, p_{l}\right) \in \mathscr{D}_{2}$, we have

$$
\begin{aligned}
& S>k_{l+1}, \\
& S \geq \sum_{j=0}^{l+1} k_{j}, \\
& S \geq \sum_{j=0}^{l} p_{j} .
\end{aligned}
$$

Let $\mathscr{D}_{3}$ be a finite subset of $\mathbb{N} \backslash\{0,1\}$. Let $M^{0}, \bar{X}_{0}>0$ be given real numbers and let $\bar{Z}_{j}^{0}>M^{0}, 0 \leq j \leq l$ be real numbers. Let

$$
\begin{gathered}
d_{\left(k_{0}, \ldots, k_{l+1}\right)}\left(Z_{0}, \ldots, Z_{l}, X\right) \in G\left(\bar{Z}_{0}^{0}, \ldots, \bar{Z}_{l}^{0}, \bar{X}^{0}\right), \\
f_{\left(p_{0}, \ldots, p_{l}\right)}\left(Z_{0}, \ldots, Z_{l}\right) \in G\left(\bar{Z}_{0}^{0}, \ldots, \bar{Z}_{l}^{0}, \bar{X}^{0}\right), \\
e_{m}\left(Z_{0}, \ldots, Z_{l}, X\right) \in G\left(\bar{Z}_{0}^{0}, \ldots, \bar{Z}_{l}^{0}, \bar{X}^{0}\right)
\end{gathered}
$$

for all $\left(k_{0}, \ldots, k_{l+1}\right) \in \mathscr{D}_{1}$, all $\left(p_{0}, \ldots, p_{l}\right) \in \mathscr{D}_{2}$, and all $m \in$ $\mathscr{D}_{3}$. For all $0 \leq j \leq S-1$, we also choose $\varphi_{j}\left(Z_{0}, \ldots, Z_{l}\right) \in$ $G\left(\bar{Z}_{0}^{0}, \ldots, \bar{Z}_{l}^{0}, \bar{X}^{0}\right)$. 
We consider the following Cauchy problem:

$$
\begin{aligned}
\partial_{X}^{S} U & \left(Z_{0}, \ldots, Z_{l}, X\right) \\
= & \sum_{\underline{k}=\left(k_{0}, \ldots, k_{l}, k_{l+1}\right) \in \mathscr{D}_{1}} d_{\underline{k}}\left(Z_{0}, \ldots, Z_{l}, X\right) \partial_{Z_{0}}^{k_{0}} \cdots \partial_{Z_{l}}^{k_{l}} \partial_{X}^{k_{l+1}} \\
& \cdot U\left(Z_{0}, \ldots, Z_{l}, X\right) \\
& +\sum_{\underline{p}=\left(p_{0}, \ldots, p_{l}\right) \in \mathscr{D}_{2}} f_{\underline{p}}\left(Z_{0}, \ldots, Z_{l}\right) \partial_{Z_{0}}^{p_{0}} \ldots \partial_{Z_{l}}^{p_{l}} \\
& \cdot\left(\sum_{m \in \mathscr{D}_{3}} e_{m}\left(Z_{0}, \ldots, Z_{l}, X\right)\left(U\left(Z_{0}, \ldots, Z_{l}, X\right)\right)^{m}\right)
\end{aligned}
$$

for given initial data

$$
\left(\partial_{X}^{j} U\right)\left(Z_{0}, \ldots, Z_{l}, 0\right)=\varphi_{j}\left(Z_{0}, \ldots, Z_{l}\right), \quad 0 \leq j \leq S-1 .
$$

Then, for given real numbers $\bar{Z}_{j}^{1}>0,0 \leq j \leq l$, with $M^{0}<$ $\bar{Z}_{j}^{1}<\bar{Z}_{j}^{0}$, one can choose $0<\bar{X}^{1}<\bar{X}^{0}$ and $\delta>0$ (which depend on $\bar{Z}_{j}^{1}$ for $0 \leq j \leq l$, on $\left\|d_{\underline{k}}\left(Z_{0}, \ldots, Z_{l}, X\right)\right\|_{\left(\bar{Z}_{0}^{0}, \ldots, \bar{Z}_{l}^{0}, \bar{X}^{0}\right)}$ for $\underline{k} \in \mathscr{D}_{1}$, on $\left\|f_{\underline{p}}\left(Z_{0}, \ldots, Z_{l}\right)\right\|_{\left(\bar{Z}_{0}^{0}, \ldots, \bar{Z}_{l}^{0}, \bar{X}^{0}\right)}$ for $\underline{p} \in \mathscr{D}_{2}$, and on $\left\|e_{m}\left(Z_{0}, \ldots, Z_{l}, X\right)\right\|_{\left(\bar{Z}_{0}^{0}, \ldots, \bar{Z}_{l}^{0}, \bar{X}^{0}\right)}$ for $\left.m \in \mathscr{D}_{3}\right)$ such that if

$$
\left\|\varphi_{j}\left(Z_{0}, \ldots, Z_{l}\right)\right\|_{\left(\bar{Z}_{0}^{0}, \ldots, \bar{Z}_{l}^{0}, \bar{X}^{0}\right)}<\delta, \quad 0 \leq j \leq S-1,
$$

then the problem (40), (41) has a unique solution $U\left(Z_{0}, \ldots\right.$, $\left.Z_{l}, X\right) \in G\left(\bar{Z}_{0}^{1}, \ldots, \bar{Z}_{l}^{1}, \bar{X}^{1}\right)$. Moreover, there exists a constant $C_{3}>0$, depending on $\bar{X}^{0}, \bar{X}^{1}, \bar{Z}_{j}^{0}, \bar{Z}_{j}^{1}$ for $0 \leq$ $j \leq l$, on $\left\|d_{\underline{k}}\left(Z_{0}, \ldots, Z_{l}, X\right)\right\|_{\left(\bar{Z}_{0}^{0}, \ldots, \bar{Z}_{l}^{0}, \bar{X}^{0}\right)}$ for $\underline{k} \in \mathscr{D}_{1}$, on $\left\|f_{\underline{p}}\left(Z_{0}, \ldots, Z_{l}\right)\right\|_{\left(\bar{Z}_{0}^{0}, \ldots, \bar{Z}_{l}^{0}, \bar{X}^{0}\right)}$ for $\underline{p} \in \mathscr{D}_{2}$, and on $\| e_{m}\left(Z_{0}, \ldots\right.$, $\left.Z_{l}, X\right) \|_{\left(\bar{Z}_{0}^{0}, \ldots, \bar{Z}_{l}^{0}, \bar{X}^{0}\right)}$ for $m \in \mathscr{D}_{3}$, such that

$$
\left\|U\left(Z_{0}, \ldots, Z_{l}, X\right)\right\|_{\left(\bar{Z}_{0}^{1}, \ldots, \bar{Z}_{l}^{1}, \bar{X}^{1}\right)} \leq \delta C_{3} .
$$

Proof. We put

$$
w\left(Z_{0}, \ldots, Z_{l}, X\right)=\sum_{j=0}^{S-1} \varphi_{j}\left(Z_{0}, \ldots, Z_{l}\right) \frac{X^{j}}{j !}
$$

and we consider the map $A: \mathbb{C}\left[\left[Z_{0}, \ldots, Z_{l}, X\right]\right] \rightarrow \mathbb{C}\left[\left[Z_{0}\right.\right.$, $\left.\ldots, Z_{l}, X\right]$ ] defined as

$$
\begin{aligned}
& A\left(H\left(Z_{0}, \ldots, Z_{l}, X\right)\right) \\
& =\sum_{\underline{k}=\left(k_{0}, \ldots, k_{l}, k_{l+1}\right) \in D_{1}} d_{\underline{k}}\left(Z_{0}, \ldots, Z_{l}, X\right) \partial_{Z_{0}}^{k_{0}} \ldots \partial_{Z_{l}}^{k_{l}} \partial_{X}^{k_{l+1}-S}
\end{aligned}
$$

$$
\begin{aligned}
& \cdot H\left(Z_{0}, \ldots, Z_{l}, X\right) \\
& +\sum_{\underline{k}=\left(k_{0}, \ldots, k_{l}, k_{l+1}\right) \in \mathscr{D}_{1}} d_{\underline{k}}\left(Z_{0}, \ldots, Z_{l}, X\right) \partial_{Z_{0}}^{k_{0}} \ldots \partial_{Z_{l}}^{k_{l}} \partial_{X}^{k_{l+1}} \\
& \cdot w\left(Z_{0}, \ldots, Z_{l}, X\right) \\
& +\sum_{\underline{p}=\left(p_{0}, \ldots, p_{l}\right) \in \mathscr{D}_{2}} f_{\underline{p}}\left(Z_{0}, \ldots, Z_{l}\right) \partial_{Z_{0}}^{p_{0}} \ldots \partial_{Z_{l}}^{p_{l}} \\
& \cdot\left(\sum_{m \in \mathscr{D}_{3}} e_{m}\left(Z_{0}, \ldots, Z_{l}, X\right)\right. \\
& \left.\cdot\left(\partial_{X}^{-S} H\left(Z_{0}, \ldots, Z_{l}, X\right)+w\left(Z_{0}, \ldots, Z_{l}, X\right)\right)^{m}\right) .
\end{aligned}
$$

Lemma 6. Let $\bar{Z}_{j}^{1}>0,0 \leq j \leq l$, be real numbers such that $M^{0}<\bar{Z}_{j}^{1}<Z_{j}^{0}$. Then, there exist $\delta>0$, a real number $0<$ $\bar{X}^{1}<\bar{X}^{0}$, and a constant $K_{4}>0$ (depending on $\bar{Z}_{j}^{1}$ for $0 \leq j \leq l$, on $\left\|d_{\underline{k}}\left(Z_{0}, \ldots, Z_{l}, X\right)\right\|_{\left(\bar{Z}_{0}^{0}, \ldots, \bar{Z}_{l}^{0}, \bar{X}^{0}\right)}$ for $\underline{k} \in \mathscr{D}_{1}$, on $\left\|f_{\underline{p}}\left(Z_{0}, \ldots, Z_{l}\right)\right\|_{\left(\bar{Z}_{0}^{0}, \ldots, \bar{Z}_{l}^{0}, \bar{X}^{0}\right)}$ for $\underline{p} \in \mathscr{D}_{2}$, and on $\| e_{m}\left(Z_{0}\right.$, $\left.\ldots, Z_{l}, X\right) \|_{\left(\bar{Z}_{0}^{0}, \ldots, \bar{Z}_{l}^{0}, \bar{X}^{0}\right)}$ for $\left.m \in \mathscr{D}_{3}\right)$, such that if one puts $R=$ $K_{4} \delta$,

(i) we have

$$
A\left(B_{R}\right) \subset B_{R}
$$

where $B_{R}$ is the closed ball of radius $R$, centered at 0 in $G\left(\bar{Z}_{0}^{1}, \ldots, \bar{Z}_{l}^{1}, \bar{X}^{1}\right)$;

(ii) for all $H_{1}, H_{2} \in B_{R}$,

$$
\left\|A\left(H_{1}\right)-A\left(H_{2}\right)\right\|_{\left(\bar{Z}_{0}^{1}, \ldots, \bar{Z}_{l}^{1}, \bar{X}^{1}\right)} \leq \frac{1}{2}\left\|H_{1}-H_{2}\right\|_{\left(\bar{Z}_{0}^{1}, \ldots, \bar{Z}_{l}^{1}, \bar{X}^{1}\right)} .
$$

Proof. We first show (i). We fix $\bar{Z}_{j}^{1}>0,0 \leq j \leq l$, be real numbers such that $M^{0}<\bar{Z}_{j}^{1}<Z_{j}^{0}$. We also consider $\delta>0$ for which (42) holds. Let $R>0$ be of the form $R=K_{4} \delta$ for some constant $K_{4}>0$. We take $H \in B_{R}$ where $B_{R}$ is the closed ball of radius $R$, centered at 0 in $G\left(\bar{Z}_{0}^{1}, \ldots, \bar{Z}_{l}^{1}, \bar{X}^{1}\right)$ for some real number $0<\bar{X}^{1}<\bar{X}^{0}$. From Lemmas 2 and 4 , we get

$$
\begin{gathered}
\| d_{\underline{k}}\left(Z_{0}, \ldots, Z_{l}, X\right) \partial_{Z_{0}}^{k_{0}} \cdots \partial_{Z_{l}}^{k_{l}} \partial_{X}^{k_{l+1}-S} \\
\cdot H\left(Z_{0}, \ldots, Z_{l}, X\right) \|_{\left(\bar{Z}_{0}^{1}, \ldots, \bar{Z}_{l}^{1}, \bar{X}^{1}\right)} \\
\leq\left\|d_{\underline{k}}\left(Z_{0}, \ldots, Z_{l}, X\right)\right\|_{\left(\bar{Z}_{0}^{1}, \ldots, \bar{Z}_{l}^{1}, \bar{X}^{1}\right)} \\
\cdot\left(\bar{Z}_{0}^{1}\right)^{-k_{0}} \cdots\left(\bar{Z}_{l}^{1}\right)^{-k_{l}}\left(\bar{X}^{1}\right)^{S-k_{l+1}}
\end{gathered}
$$


8

Abstract and Applied Analysis

$$
\begin{aligned}
& \cdot\left\|H\left(Z_{0}, \ldots, Z_{l}, X\right)\right\|_{\left(\bar{Z}_{0}^{1}, \ldots, \bar{Z}_{l}^{1}, \bar{X}^{1}\right)} \\
\leq & \left\|d_{\underline{k}}\left(Z_{0}, \ldots, Z_{l}, X\right)\right\|_{\left(\bar{Z}_{0}^{0}, \ldots, \bar{Z}_{l}^{0}, \bar{X}^{0}\right)} \\
& \cdot\left(\bar{Z}_{0}^{1}\right)^{-k_{0}} \ldots\left(\bar{Z}_{l}^{1}\right)^{-k_{l}} \times\left(\bar{X}^{1}\right)^{S-k_{l+1}} R .
\end{aligned}
$$

Now, from Lemmas 3 and 4 , we get a constant $C_{4}>0$ (depending on $k_{0}, \ldots, k_{l}, \bar{X}^{0}, \bar{X}^{1}, \bar{Z}_{j}^{0}, \bar{Z}_{j}^{1}$, for $0 \leq j \leq l$ ) with

$$
\begin{gathered}
\left\|d_{\underline{k}}\left(Z_{0}, \ldots, Z_{l}, X\right)\left(\partial_{Z_{0}}^{k_{0}} \ldots \partial_{Z_{l}}^{k_{l}} \varphi_{j}\right)\left(Z_{0}, \ldots, Z_{l}\right) \frac{X^{q}}{q !}\right\|_{\left(\bar{Z}_{0}^{1}, \ldots, \bar{Z}_{l}^{1}, \bar{X}^{1}\right)} \\
\leq\left\|d_{\underline{k}}\left(Z_{0}, \ldots, Z_{l}, X\right)\right\|_{\left(\bar{Z}_{0}^{1}, \ldots, \bar{Z}_{l}^{1}, \bar{X}^{1}\right)}\left\|\frac{X^{q}}{q !}\right\|_{\left(\bar{Z}_{0}^{1}, \ldots, \bar{Z}_{l}^{1}, \bar{X}^{1}\right)} \\
\quad \cdot C_{4}\left(\bar{Z}_{0}^{1}\right)^{-k_{0}} \ldots\left(\bar{Z}_{l}^{1}\right)^{-k_{l}} \times\left\|\varphi_{j}\left(Z_{0}, \ldots, Z_{l}\right)\right\|_{\left(\bar{Z}_{0}^{0}, \ldots, \bar{Z}_{l}^{0}, \bar{X}^{0}\right)} \\
\leq\left\|d_{\underline{k}}\left(Z_{0}, \ldots, Z_{l}, X\right)\right\|_{\left(\bar{Z}_{0}^{0}, \ldots, \bar{Z}_{l}^{0}, \bar{X}^{0}\right)}\left\|\frac{X^{q}}{q !}\right\|_{\left(\bar{Z}_{0}^{0}, \ldots, \bar{Z}_{l}^{0}, \bar{X}^{0}\right)} \\
\cdot C_{4}\left(\bar{Z}_{0}^{1}\right)^{-k_{0}} \ldots\left(\bar{Z}_{l}^{1}\right)^{-k_{l}} \delta
\end{gathered}
$$

for all $0 \leq j, q \leq S-1$.

Using Leibniz formula, we deduce from Lemma 4 that

$$
\begin{aligned}
& \| f_{\underline{p}}\left(Z_{0}, \ldots, Z_{p}\right) \partial_{Z_{0}}^{p_{0}} \cdots \partial_{Z_{l}}^{p_{l}} \\
& \cdot\left(e_{m}\left(Z_{0}, \ldots, Z_{l}, X\right)\right. \\
& \left.\quad \cdot\left(\partial_{X}^{-S} H\left(Z_{0}, \ldots, Z_{l}, X\right)+w\left(Z_{0}, \ldots, Z_{l}, X\right)\right)^{m}\right) \|_{\left(\bar{Z}_{0}^{1}, \ldots, \bar{Z}_{l}^{1}, \bar{X}^{1}\right)} \\
& \leq \sum_{\sum_{j=0}^{m} p_{h, j}=p_{h}, 0 \leq h \leq l} \frac{p_{0} ! \cdots p_{l} !}{\prod_{0 \leq j \leq m, 0 \leq h \leq l} p_{h, j} !} \\
& \quad \cdot\left\|f_{\underline{p}}\left(Z_{0}, \ldots, Z_{p}\right)\right\|_{\left(\bar{Z}_{0}^{1}, \ldots, \bar{Z}_{l}^{1}, \bar{X}^{1}\right)} \\
& \quad\left\|\partial_{Z_{0}}^{p_{0,0}} \ldots \partial_{Z_{l}}^{p_{l, 0}} e_{m}\left(Z_{0}, \ldots, Z_{l}, X\right)\right\|_{\left(\bar{Z}_{0}^{1}, \ldots, \bar{Z}_{l}^{1}, \bar{X}^{1}\right)} \\
& \quad \prod_{j=1}^{m} \|\left(\partial_{Z_{0}}^{p_{0, j}} \ldots \partial_{Z_{l}}^{p_{l, j}}\right)\left(\partial_{X}^{-S} H\left(Z_{0}, \ldots, Z_{l}, X\right)\right. \\
& \left.\quad+w\left(Z_{0}, \ldots, Z_{l}, X\right)\right) \|_{\left(\bar{Z}_{0}^{1}, \ldots, \bar{Z}_{l}^{1}, \bar{X}^{1}\right)} \cdot
\end{aligned}
$$

By Lemma 3, one also gets a constant $C_{4.1}>0$ (depending on $p_{0,0}, \ldots, p_{l, 0}, \bar{X}^{0}, \bar{X}^{1}, \bar{Z}_{j}^{0}, \bar{Z}_{j}^{1}$ for $\left.0 \leq j \leq l\right)$ with

$$
\begin{aligned}
& \left\|\partial_{Z_{0}}^{p_{0,0}} \ldots \partial_{Z_{l}}^{p_{l, 0}} e_{m}\left(Z_{0}, \ldots, Z_{l}, X\right)\right\|_{\left(\bar{Z}_{0}^{1}, \ldots, \bar{Z}_{l}^{1}, \bar{X}^{1}\right)} \\
& \leq C_{4.1}\left(\bar{Z}_{0}^{1}\right)^{-p_{0,0}} \cdots\left(\bar{Z}_{l}^{1}\right)^{-p_{l, 0}}\left\|e_{m}\left(Z_{0}, \ldots, Z_{l}, X\right)\right\|_{\left(\bar{Z}_{0}^{0}, \ldots, \bar{Z}_{l}^{0}, \bar{X}^{0}\right)} \cdot \quad \leq K_{4} \delta .
\end{aligned}
$$

By Lemma 2, one finds

$$
\begin{aligned}
& \left\|\partial_{Z_{0}}^{p_{0, j}} \cdots \partial_{Z_{l}}^{p_{l, j}} \partial_{X}^{-S} H\left(Z_{0}, \ldots, Z_{l}, X\right)\right\|_{\left(\bar{Z}_{0}^{1}, \ldots, \bar{Z}_{l}^{1}, \bar{X}^{1}\right)} \\
& \leq\left(\bar{Z}_{0}^{1}\right)^{-p_{0, j}} \cdots\left(\bar{Z}_{l}^{1}\right)^{-p_{l, j}}\left(\bar{X}^{1}\right)^{S}\left\|H\left(Z_{0}, \ldots, Z_{l}, X\right)\right\|_{\left(\bar{Z}_{0}^{1}, \ldots, \bar{Z}_{l}^{1}, \bar{X}^{1}\right)} \\
& \leq\left(\bar{Z}_{0}^{1}\right)^{-p_{0, j}} \cdots\left(\bar{Z}_{l}^{1}\right)^{-p_{l, j}}\left(\bar{X}^{1}\right)^{S} R .
\end{aligned}
$$

Due to Lemma 3, we get a constant $C_{4.2}>0$ (depending on $p_{0, j}, \ldots, p_{l, j}, \bar{X}^{0}, \bar{X}^{1}, \bar{Z}_{m}^{0}, \bar{Z}_{m}^{1}$ for $\left.0 \leq m \leq l\right)$ such that

$$
\begin{aligned}
& \left\|\partial_{Z_{0}}^{p_{0, j}} \cdots \partial_{Z_{l}}^{p_{l, j}} w\left(Z_{0}, \ldots, Z_{l}, X\right)\right\|_{\left(\bar{Z}_{0}^{1}, \ldots, \bar{Z}_{l}^{1}, \bar{X}^{1}\right)} \\
& \leq \sum_{q=0}^{S-1}\left\|\partial_{Z_{0}}^{p_{0, j}} \ldots \partial_{Z_{l}}^{p_{l, j}} \varphi_{q}\left(Z_{0}, \ldots, Z_{l}\right)\right\|_{\left(\bar{Z}_{0}^{1}, \ldots, \bar{Z}_{l}^{1}, \bar{X}^{1}\right)}\left\|\frac{X^{q}}{q !}\right\|_{\left(\bar{Z}_{0}^{1}, \ldots, \bar{Z}_{l}^{1}, \bar{X}^{1}\right)} \\
& \leq C_{4.2} \sum_{q=0}^{S-1}\left(\bar{Z}_{0}^{1}\right)^{-p_{0, j}} \cdots\left(\bar{Z}_{l}^{1}\right)^{-p_{l, j}}\left\|\varphi_{q}\left(Z_{0}, \ldots, Z_{l}\right)\right\|_{\left(\bar{Z}_{0}^{0}, \ldots, \bar{Z}_{l}^{0}, \bar{X}^{0}\right)} \\
& \quad\left\|\frac{X^{q}}{q !}\right\|_{\left(\bar{Z}_{0}^{0}, \ldots, \bar{Z}_{l}^{0}, \bar{X}^{0}\right)} \\
& \leq C_{4.2} \sum_{q=0}^{S-1}\left(\bar{Z}_{0}^{1}\right)^{-p_{0, j}} \cdots\left(\bar{Z}_{l}^{1}\right)^{-p_{l, j}} \delta\left\|\frac{X^{q}}{q !}\right\|_{\left(\bar{Z}_{0}^{0}, \ldots, \bar{Z}_{l}^{0}, \bar{X}^{0}\right)} .
\end{aligned}
$$

Now, we can choose $0<\bar{X}^{1}<\bar{X}^{0}, \delta>0$ and the constant $K_{4}>0$ (recall that $R=K_{4} \delta$ ) in such a way that

$$
\sum_{\underline{k}=\left(k_{0}, \ldots, k_{l+1}\right) \in \mathscr{D}_{1}}\left\|d_{\underline{k}}\left(Z_{0}, \ldots, Z_{l}, X\right)\right\|_{\left(\bar{Z}_{0}^{0}, \ldots, \bar{Z}_{l}^{0}, \bar{X}^{0}\right)}
$$

$$
\begin{gathered}
\cdot\left(\bar{Z}_{0}^{1}\right)^{-k_{0}} \ldots\left(\bar{Z}_{l}^{1}\right)^{-k_{l}} \\
\cdot\left(\left(\bar{X}^{1}\right)^{S-k_{l+1}} K_{4} \delta+\sum_{q=0}^{S-1}\left\|\frac{X^{q}}{q !}\right\|_{\left(\bar{Z}_{0}^{0}, \ldots, \bar{Z}_{l}^{0}, \bar{X}^{0}\right)} C_{4} \delta\right) \\
+\sum_{\underline{p}=\left(p_{0}, \ldots, p_{l}\right) \in \mathscr{D}_{2}} \sum_{m \in \mathscr{D}_{3} \sum_{j=0}^{m} p_{h, j}=p_{h}, 0 \leq h \leq l} \frac{p_{0} ! \cdots p_{l} !}{\prod_{0 \leq j \leq m, 0 \leq h \leq l} p_{h, j} !} \\
\cdot\left\|f_{\underline{p}}\left(Z_{0}, \ldots, Z_{l}\right)\right\|_{\left(\bar{Z}_{0}^{0}, \ldots, \bar{Z}_{l}^{0}, \bar{X}^{0}\right)} \times C_{4.1}\left(\bar{Z}_{0}^{1}\right)^{-p_{0,0}} \ldots\left(\bar{Z}_{l}^{1}\right)^{-p_{l, 0}} \\
\cdot\left\|e_{m}\left(Z_{0}, \ldots, Z_{l}, X\right)\right\|_{\left(\bar{Z}_{0}^{0}, \ldots, \bar{Z}_{l}^{0}, \bar{X}^{0}\right)} \\
\cdot \prod_{j=1}^{m}\left(\left(\bar{Z}_{0}^{1}\right)^{-p_{0, j}} \ldots\left(\bar{Z}_{l}^{1}\right)^{-p_{l, j}}\left(\bar{X}^{1}\right)^{S} K_{4} \delta\right. \\
\left.+C_{4.2} \sum_{q=0}^{S-1}\left(\bar{Z}_{0}^{1}\right)^{-p_{0, j}} \ldots\left(\bar{Z}_{l}^{1}\right)^{-p_{l, j}} \delta\left\|\frac{X^{q}}{q !}\right\|_{\left(\bar{Z}_{0}^{0}, \ldots, \bar{Z}_{l}^{0}, \bar{X}^{0}\right)}\right)
\end{gathered}
$$


Hence, gathering (48), (49), (50), (51), (52), (53), and (54) yields inclusion (46).

We turn to the proof of (ii). As above, we fix $\bar{Z}_{j}^{1}>0,0 \leq$ $j \leq l$, be real numbers such that $M^{0}<\bar{Z}_{j}^{1}<Z_{j}^{0}$. We also consider $\delta>0$ for which (42) holds. Let $R>0$ be of the form $R=K_{4} \delta$ for some constant $K_{4}>0$. We take $H_{1}, H_{2} \in$ $B_{R}$ where $B_{R}$ is the closed ball of radius $R$, centered at 0 in $G\left(\bar{Z}_{0}^{1}, \ldots, \bar{Z}_{l}^{1}, \bar{X}^{1}\right)$ for some real number $0<\bar{X}^{1}<\bar{X}^{0}$. From Lemmas 2 and 4 , we get that

$$
\begin{aligned}
\| & \| d_{\underline{k}}\left(Z_{0}, \ldots, Z_{l}, X\right) \partial_{Z_{0}}^{k_{0}} \ldots \partial_{Z_{l}}^{k_{l}} \partial_{X}^{k_{l+1}-S} \\
& \cdot\left(H_{2}\left(Z_{0}, \ldots, Z_{l}, X\right)-H_{1}\left(Z_{0}, \ldots, Z_{l}, X\right)\right) \|_{\left(\bar{Z}_{0}^{1}, \ldots, \bar{Z}_{l}^{1}, \bar{X}^{1}\right)} \\
\leq & \left\|d_{\underline{k}}\left(Z_{0}, \ldots, Z_{l}, X\right)\right\|_{\left(\bar{Z}_{0}^{1}, \ldots, \bar{Z}_{l}^{1}, \bar{X}^{1}\right)} \\
& \cdot\left(\bar{Z}_{0}^{1}\right)^{-k_{0}} \ldots\left(\bar{Z}_{l}^{1}\right)^{-k_{l}}\left(\bar{X}^{1}\right)^{S-k_{l+1}} \\
& \cdot\left\|H_{2}\left(Z_{0}, \ldots, Z_{l}, X\right)-H_{1}\left(Z_{0}, \ldots, Z_{l}, X\right)\right\|_{\left(\bar{Z}_{0}^{1}, \ldots, \bar{Z}_{l}^{1}, \bar{X}^{1}\right)} \\
\leq & \left\|d_{\underline{k}}\left(Z_{0}, \ldots, Z_{l}, X\right)\right\|_{\left(\bar{Z}_{0}^{0}, \ldots, \bar{Z}_{l}^{0}, \bar{X}^{0}\right)} \\
& \cdot\left(\bar{Z}_{0}^{1}\right)^{-k_{0}} \ldots\left(\bar{Z}_{l}^{1}\right)^{-k_{l}}\left(\bar{X}^{1}\right)^{S-k_{l+1}} \\
& \cdot\left\|H_{2}\left(Z_{0}, \ldots, Z_{l}, X\right)-H_{1}\left(Z_{0}, \ldots, Z_{l}, X\right)\right\|_{\left(\bar{Z}_{0}^{1}, \ldots, \bar{Z}_{l}^{1}, \bar{X}^{1}\right)} .
\end{aligned}
$$

Using the identity $b^{m}-a^{m}=(b-a) \sum_{s=0}^{m-1} a^{s} b^{m-1-s}$ for any complex numbers $a, b$ and any integer $m \geq 2$, we can write

$$
\begin{aligned}
\left(\partial_{X}^{-S} H_{1}\left(Z_{0}, \ldots, Z_{l}, X\right)+w\left(Z_{0}, \ldots, Z_{l}, X\right)\right)^{m} \\
-\partial_{X}^{-S} H_{2}\left(Z_{0}, \ldots, Z_{l}, X\right)+w\left(Z_{0}, \ldots, Z_{l}, X\right)^{m} \\
=\left(\partial_{X}^{-S} H_{1}-\partial_{X}^{-S} H_{2}\right) \\
\quad \cdot \sum_{s=0}^{m-1}\left(\partial_{X}^{-S} H_{2}+w\right)^{s}\left(\partial_{X}^{-S} H_{1}+w\right)^{m-1-s} .
\end{aligned}
$$

Using Leibniz formula, we deduce from Lemma 4 that

$$
\begin{aligned}
& \| f_{\underline{p}}\left(Z_{0}, \ldots, Z_{l}\right) \partial_{Z_{0}}^{p_{0}} \cdots \partial_{Z_{l}}^{p_{l}} e_{m} \\
& \cdot\left(Z_{0}, \ldots, Z_{l}, X\right)\left(\partial_{X}^{-S} H_{1}-\partial_{X}^{-S} H_{2}\right) \\
& \quad \cdot\left(\sum_{s=0}^{m-1}\left(\partial_{X}^{-S} H_{2}+w\right)^{s}\left(\partial_{X}^{-S} H_{1}+w\right)^{m-1-s}\right) \|_{\left(\bar{Z}_{0}^{1}, \ldots, \bar{Z}_{l}^{1}, \bar{X}^{1}\right)} \\
& \leq \sum_{\sum_{j=0}^{2} p_{h, j}=p_{h}, 0 \leq h \leq l} \frac{p_{0} ! \cdots p_{l} !}{\prod_{0 \leq j \leq 2,0 \leq h \leq l} p_{h, j} !}\left\|f_{\underline{p}}\left(Z_{0}, \ldots, Z_{l}\right)\right\|_{\left(\overline{(}_{0}^{1}, \ldots, \bar{Z}_{l}^{1}, \bar{X}^{1}\right)} \\
& \quad \cdot\left\|\partial_{Z_{0}, \ldots}^{p_{0,0}} \ldots \partial_{Z_{l}}^{p_{l, 0}} e_{m}\left(Z_{0}, \ldots, Z_{l}, X\right)\right\|_{\left(\bar{Z}_{0}^{1}, \ldots, \bar{Z}_{l}^{1}, \bar{X}^{1}\right)}
\end{aligned}
$$

$$
\begin{aligned}
& \cdot\left\|\partial_{Z_{0}}^{p_{0,1}} \cdots \partial_{Z_{l}}^{p_{l, 1}}\left(\partial_{X}^{-S} H_{1}-\partial_{X}^{-S} H_{2}\right)\right\|_{\left(\bar{Z}_{0}^{1}, \ldots, \bar{Z}_{l}^{1}, \bar{X}^{1}\right)} \\
& \cdot \| \partial_{Z_{0}}^{p_{0,2}} \cdots \partial_{Z_{l}}^{p_{l, 2}} \\
& \cdot\left(\sum_{s=0}^{m-1}\left(\partial_{X}^{-S} H_{2}+w\right)^{s}\left(\partial_{X}^{-S} H_{1}+w\right)^{m-1-s}\right) \|_{\left(\bar{Z}_{0}^{1}, \ldots, \bar{Z}_{l}^{1}, \bar{X}^{1}\right)} .
\end{aligned}
$$

By Lemma 3, one also gets a constant $C_{4.3}>0$ (depending on $p_{0,0}, \ldots, p_{l, 0}, \bar{X}^{0}, \bar{X}^{1}, \bar{Z}_{j}^{0}, \bar{Z}_{j}^{1}$ for $\left.0 \leq j \leq l\right)$ with

$$
\begin{aligned}
& \left\|\partial_{Z_{0}}^{p_{0,0}} \cdots \partial_{Z_{l}}^{p_{l, 0}} e_{m}\left(Z_{0}, \ldots, Z_{l}, X\right)\right\|_{\left(\bar{Z}_{0}^{1}, \ldots, \bar{Z}_{l}^{1}, \bar{X}^{1}\right)} \\
& \leq C_{4.3}\left(\bar{Z}_{0}^{1}\right)^{-p_{0,0}} \cdots\left(\bar{Z}_{l}^{1}\right)^{-p_{l, 0}}\left\|e_{m}\left(Z_{0}, \ldots, Z_{l}, X\right)\right\|_{\left(\bar{Z}_{0}^{0}, \ldots, \bar{Z}_{l}^{0}, \bar{X}^{0}\right)} .
\end{aligned}
$$

By Lemma 2, one finds

$$
\begin{aligned}
& \left\|\partial_{Z_{0}}^{p_{0,1}} \cdots \partial_{Z_{l}}^{p_{l, 1}}\left(\partial_{X}^{-S} H_{1}-\partial_{X}^{-S} H_{2}\right)\right\|_{\left(\bar{Z}_{0}^{1}, \ldots, \bar{Z}_{l}^{1}, \bar{X}^{1}\right)} \\
& \quad \leq\left(\bar{Z}_{0}^{1}\right)^{-p_{0,1}} \cdots\left(\bar{Z}_{l}^{1}\right)^{-p_{l, 1}}\left(\bar{X}^{1}\right)^{S}\left\|H_{1}-H_{2}\right\|_{\left(\bar{Z}_{0}^{1}, \ldots, \bar{Z}_{l}^{1}, \bar{X}^{1}\right)} .
\end{aligned}
$$

Using again Leibniz formula and Lemma 4, we can write

$$
\begin{aligned}
\| \partial_{Z_{0}}^{p_{0,2}} \cdots & \partial_{Z_{l}}^{p_{l, 2}}\left(\left(\partial_{X}^{-S} H_{2}+w\right)^{s}\left(\partial_{X}^{-S} H_{1}+w\right)^{m-1-s}\right) \|_{\left(\bar{Z}_{0}^{1}, \ldots, \bar{Z}_{l}^{1}, \bar{X}^{1}\right)} \\
\leq & \sum_{\sum_{j=1}^{m-1} p_{h, 2, j}=p_{h, 2}, 0 \leq h \leq l} \frac{p_{0,2} ! \cdots p_{l, 2} !}{\prod_{1 \leq j \leq m-1,0 \leq h \leq l} p_{h, 2, j} !} \\
& \cdot \prod_{j=1}^{s}\left\|\left(\partial_{Z_{0}}^{p_{0,2, j}} \cdots \partial_{Z_{l}}^{p_{l, 0, j}}\right)\left(\partial_{X}^{-S} H_{2}+w\right)\right\|_{\left(\bar{Z}_{0}^{1}, \ldots, \bar{Z}_{l}^{1}, \bar{X}^{1}\right)} \\
& \cdot \prod_{j=s+1}^{m-1}\left\|\left(\partial_{Z_{0}}^{p_{0,2, j}} \cdots \partial_{Z_{l}}^{p_{l, 0, j}}\right)\left(\partial_{X}^{-S} H_{1}+w\right)\right\|_{\left(\bar{Z}_{0}^{1}, \ldots, \bar{Z}_{l}^{1}, \bar{X}^{1}\right)}
\end{aligned}
$$

By Lemmas 2 and 3, one finds a constant $C_{4.4}>0$ (depending on $p_{0,2, j}, \ldots, p_{l, 2, j}, \bar{X}^{0}, \bar{X}^{1}, \bar{Z}_{j}^{0}, \bar{Z}_{j}^{1}$ for $0 \leq j \leq l$ ) such that

$$
\begin{aligned}
& \left\|\left(\partial_{Z_{0}}^{p_{0,2, j}} \cdots \partial_{Z_{l}}^{p_{l, 2, j}}\right)\left(\partial_{X}^{-S} H_{r}+w\right)\right\|_{\left(\bar{Z}_{0}^{1}, \ldots, \bar{Z}_{l}^{1}, \bar{X}^{1}\right)} \\
& \leq\left(\bar{Z}_{0}^{1}\right)^{-p_{0,2, j}} \cdots\left(\bar{Z}_{l}^{1}\right)^{-p_{l, 2, j}}\left(\bar{X}^{1}\right)^{S} R \\
& \quad+C_{4.4} \sum_{q=0}^{S-1}\left(\bar{Z}_{0}^{1}\right)^{-p_{0,2, j}} \cdots\left(\bar{Z}_{l}^{1}\right)^{-p_{l, 2, j}} \delta\left\|\frac{X^{q}}{q !}\right\|_{\left(\bar{Z}_{0}^{0}, \ldots, \bar{Z}_{l}^{0}, \bar{X}^{0}\right)}
\end{aligned}
$$

for $r=1,2$. 
In the following, we choose $0<\bar{X}^{1}<\bar{X}^{0}$ in order that $\sum_{\underline{k}=\left(k_{0}, \ldots, k_{l+1}\right) \in \mathscr{D}_{1}}\left\|d_{\underline{k}}\left(Z_{0}, \ldots, Z_{l}, X\right)\right\|_{\left(\bar{Z}_{0}^{0}, \ldots, \bar{Z}_{l}^{0}, \bar{X}^{0}\right)}$

$$
\begin{gathered}
\cdot\left(\bar{Z}_{0}^{1}\right)^{-k_{0}} \cdots\left(\bar{Z}_{l}^{1}\right)^{-k_{l}}\left(\bar{X}^{1}\right)^{S-k_{l+1}} \\
+\sum_{\underline{p}=\left(p_{0}, \ldots, p_{l}\right) \in \mathscr{D}_{2}} \sum_{m \in \mathscr{D}_{3}} \sum_{\sum_{j=0}^{2} p_{p_{h, j}=p_{h}, 0 \leq h \leq l} \frac{p_{0} ! \cdots p_{l} !}{\Pi_{0 \leq j \leq 2,0 \leq h \leq l} p_{h, j} !}} \\
\cdot\left\|f_{\underline{p}}\left(Z_{0}, \ldots, Z_{l}\right)\right\|_{\left(\bar{Z}_{0}^{0}, \ldots, \bar{Z}_{l}^{0}, \bar{X}^{0}\right)}
\end{gathered}
$$$$
\cdot C_{4.3}\left(\bar{Z}_{0}^{1}\right)^{-p_{0,0}} \cdots\left(\bar{Z}_{l}^{1}\right)^{-p_{l, 0}}\left\|e_{m}\left(Z_{0}, \ldots, Z_{l}, X\right)\right\|_{\left(\bar{Z}_{0}^{0}, \ldots, \bar{Z}_{l}^{0}, \bar{X}^{0}\right)}
$$$$
\cdot\left(\bar{Z}_{0}^{1}\right)^{-p_{0,1}} \cdots\left(\bar{Z}_{l}^{1}\right)^{-p_{l, 1}}\left(\bar{X}^{1}\right)^{S}
$$$$
\cdot\left(\sum_{s=0}^{m-1} \sum_{\sum_{j=1}^{m-1} p_{h, 2, j}=p_{h, 2}, 0 \leq h \leq l} \frac{p_{0,2} ! \cdots p_{l, 2} !}{\Pi_{1 \leq j \leq m-1,0 \leq h \leq l} p_{h, 2, j} !}\right.
$$$$
\cdot \prod_{j=1}^{s}\left(\left(\bar{Z}_{0}^{1}\right)^{-p_{0,2, j}} \cdots\left(\bar{Z}_{l}^{1}\right)^{-p_{l, 2, j}}\left(\bar{X}^{1}\right)^{S} K_{4} \delta\right.
$$$$
+C_{4.4} \sum_{q=0}^{S-1}\left(\bar{Z}_{0}^{1}\right)^{-p_{0,2, j}} \cdots\left(\bar{Z}_{l}^{1}\right)^{-p_{l, 2, j}}
$$$$
\left.\cdot \delta\left\|\frac{X^{q}}{q !}\right\|_{\left(\bar{Z}_{0}^{0}, \ldots, \bar{Z}_{l}^{0}, \bar{X}^{0}\right)}\right)
$$$$
\cdot \prod_{j=s+1}^{m-1}\left(\left(\bar{Z}_{0}^{1}\right)^{-p_{0,2, j}} \cdots\left(\bar{Z}_{l}^{1}\right)^{-p_{l, 2, j}}\left(\bar{X}^{1}\right)^{S} K_{4} \delta\right.
$$$$
+C_{4.4} \sum_{q=0}^{S-1}\left(\bar{Z}_{0}^{1}\right)^{-p_{0,2, j}} \cdots\left(\bar{Z}_{l}^{1}\right)^{-p_{l, 2, j}}
$$$$
\left.\left.\cdot \delta\left\|\frac{X^{q}}{q !}\right\|_{\left(\bar{Z}_{0}^{0}, \ldots, \bar{Z}_{l}^{0}, \bar{X}^{0}\right)}\right)\right) \leq \frac{1}{2} .
$$

Taking into account all inequalities (55), (56), (57), (58), (59), (60), and (61) under constraint (62), we deduce (47).

Finally, for fixed real numbers $\bar{Z}_{j}^{1}>0,0 \leq j \leq l$, such that $M^{0}<\bar{Z}_{j}^{1}<Z_{j}^{0}$, we choose $0<\bar{X}^{1}<\bar{X}^{0}, \delta>0$ and the constant $K_{4}>0$ (recall that $R=K_{4} \delta$ ) in such a way that both constraints (54) and (62) hold. For these constants, the map $A$ satisfies both (46) and (47).

We are in a position to give the proof of Proposition 5. Let $\bar{Z}_{j}^{1}>0,0 \leq j \leq l$, be real numbers such that $M^{0}<\bar{Z}_{j}^{1}<Z_{j}^{0}$; we choose $0<\bar{X}^{1}<\bar{X}^{0}, \delta>0$ and the constant $K_{4}>0$ as in Lemma 6. We have put $R=\delta K_{4}$.
From the fact that $G\left(\bar{Z}_{0}^{1}, \ldots, \bar{Z}_{l}^{1}, \bar{X}^{1}\right)$ equipped with the norm $\|\cdot\|_{\left(\bar{Z}_{0}^{1}, \ldots, \bar{Z}_{1}^{1}, \bar{X}^{1}\right)}$ is a Banach space, the closed ball $\left(B_{R}, d\right)$ for the metric $d(x, y)=\|y-x\|_{\left(\bar{Z}_{0}^{1}, \ldots, \bar{Z}_{l}^{1}, \bar{X}^{1}\right)}$ is a complete metric space. From Lemma 6 , the map $A$ is a contraction from $\left(B_{R}, d\right)$ into itself. From the classical fixed point theorem, we deduce that there exists a unique $H_{f}\left(Z_{0}, \ldots, Z_{l}, X\right) \in B_{R}$ such that $A\left(H_{f}\right)=H_{f}$.

By construction and taking into account Lemma 2, the formal series

$$
\begin{aligned}
& U_{f}\left(Z_{0}, \ldots, Z_{l}, X\right) \\
& \quad=\partial_{X}^{-S} H_{f}\left(Z_{0}, \ldots, Z_{l}, X\right)+w\left(Z_{0}, \ldots, Z_{l}, X\right)
\end{aligned}
$$

is a solution of the problem (40), (41). Moreover, $U_{f}\left(Z_{0}, \ldots\right.$, $\left.Z_{l}, X\right) \in G\left(\bar{Z}_{0}^{1}, \ldots, \bar{Z}_{l}^{1}, \bar{X}^{1}\right)$ and

$$
\begin{aligned}
& \left\|U_{f}\left(Z_{0}, \ldots, Z_{l}, X\right)\right\|_{\left(\bar{Z}_{0}^{1}, \ldots, \bar{Z}_{l}^{1}, \bar{X}^{1}\right)} \\
& \quad \leq \delta\left(\left(\bar{X}^{1}\right)^{S} K_{4}+\sum_{j=0}^{S-1}\left\|\frac{X^{j}}{j !}\right\|_{\left(\bar{Z}_{0}^{0}, \ldots, \bar{Z}_{l}^{0}, \bar{X}^{0}\right)}\right)
\end{aligned}
$$

which yields (43). Thus proof of Proposition 5 is complete.

2.2. Weighted Banach Spaces of Holomorphic Functions on Sectors. We denote by $D(0, r)$ the open disc centered at 0 with radius $r>0$ in $\mathbb{C}$. Let $\epsilon_{0}>0$ be a real number and let $S_{d}$ be an unbounded sector in direction $d \in \mathbb{R}$ centered at 0 in $\mathbb{C}$. By convention, these sectors do not contain the origin in $\mathbb{C}$. For any open set $\mathscr{D} \subset \mathbb{C}$, we denote by $\mathcal{O}(\mathscr{D})$ the vector space of holomorphic functions on $\mathscr{D}$. Let $l \geq 1$ be an integer. For all tuples $\underline{\beta}=\left(\beta_{0}, \ldots, \beta_{l+1}\right) \in \mathbb{N}^{l+2}$, let $\rho_{\beta} \geq 0$ be positive real numbers such that $\rho_{\beta^{\prime}} \leq \rho_{\beta}$ if $\left|\beta^{\prime}\right| \geq|\underline{\beta}|$ (where by definition $\left.|\underline{\beta}|=\sum_{j=0}^{l+1} \beta_{j}\right)$. We define $\Omega_{\underline{\beta}}=D\left(0, \rho_{\underline{\beta}}\right) \cup S_{d}$.

Definition 7. Let $b>1$ be a real number and let $r_{b}(\beta)=$ $\sum_{n=0}^{|\beta|} 1 /(n+1)^{b}$ for all tuples $\beta \in \mathbb{N}^{l+2}$. Let $\epsilon \in D\left(0, \epsilon_{0}\right) \backslash\{0\}$ and $r, \sigma>0$ be real numbers. We denote by $E_{\underline{\beta}, \epsilon, \sigma, r, \Omega_{\beta}}$ the vector space of all functions $v \in \mathcal{O}\left(\Omega_{\underline{\beta}}\right)$ such that

$$
\begin{aligned}
& \|v(\tau)\|_{\underline{\beta}, \epsilon, \sigma, r, \Omega_{\underline{\beta}}} \\
& \quad:=\sup _{\tau \in \Omega_{\underline{\beta}}}|v(\tau)|\left(1+\frac{|\tau|^{2}}{|\epsilon|^{2 r}}\right) \exp \left(-\frac{\sigma}{|\epsilon|^{r}} r_{b}(\underline{\beta})|\tau|\right)
\end{aligned}
$$

is finite.

Remark 8. These norms are appropriate modifications of the norms defined in $[4,17]$.

In the next proposition, we study some parameter depending linear operators acting on the spaces $E_{\beta, \epsilon, \sigma, r, \Omega_{\beta}}$.

Proposition 9. Let $s_{1}, k_{0} \geq 0$ be integers. Let $\underline{\beta}=\left(\beta_{0}, \ldots\right.$, $\left.\beta_{l+1}\right) \in \mathbb{N}^{l+2}, \underline{\widetilde{\beta}}=\left(\widetilde{\beta}_{0}, \ldots, \widetilde{\beta}_{l+1}\right) \in \mathbb{N}^{l+2}$. Under the assumption 
that $|\widetilde{\beta}|>|\underline{\beta}|$, the operator $\tau^{s_{1}} \partial_{\tau}^{-k_{0}}$ defines a bounded linear

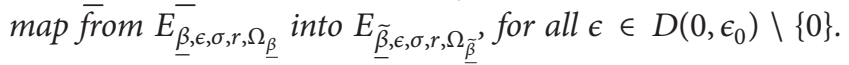
Moreover, one has

$$
\begin{aligned}
& \left\|\tau^{s_{1}} \partial_{\tau}^{-k_{0}} V_{\underline{\beta}}(\tau, \epsilon)\right\|_{\underline{\tilde{\beta}}, \epsilon, \sigma, r, \Omega_{\underline{\tilde{\beta}}}} \\
& \leq\left\|V_{\underline{\beta}}(\tau, \epsilon)\right\|_{\underline{\beta}, \epsilon, \sigma, r, \Omega_{\underline{\beta}}} \\
& \cdot|\epsilon|^{r\left(s_{1}+k_{0}\right)}\left((|\underline{\beta}|+1)^{b\left(s_{1}+k_{0}\right)}\left(\frac{\left(s_{1}+k_{0}\right) e^{-1}}{\sigma(|\widetilde{\beta}|-|\underline{\beta}|)}\right)^{s_{1}+k_{0}}\right. \\
& +(|\underline{\tilde{\beta}}|+1)^{b\left(s_{1}+k_{0}+2\right)} \\
& \left.\cdot\left(\frac{\left(s_{1}+k_{0}+2\right) e^{-1}}{\sigma(|\widetilde{\beta}|-|\underline{\beta}|)}\right)^{s_{1}+k_{0}+2}\right)
\end{aligned}
$$

for all $V_{\underline{\beta}}(\tau, \epsilon) \in E_{\underline{\beta}, \epsilon, \sigma, r, \Omega_{\beta}}$.

Proof. The proof follows the same lines of arguments as Lemma 1 from [17]. By construction of the operator $\partial_{\tau}^{-k_{0}}$, one can write

$$
\begin{aligned}
& \tau^{s_{1}} \partial_{\tau}^{-k_{0}} V_{\underline{\beta}}(\tau, \epsilon) \\
&=\tau^{s_{1}+k_{0}} \int_{0}^{1} \cdots \int_{0}^{1} V_{\underline{\beta}}\left(h_{k_{0}} \cdots h_{1} \tau, \epsilon\right) \\
& \cdot\left(1+\frac{\left|h_{k_{0}} \cdots h_{1} \tau\right|^{2}}{|\epsilon|^{2 r}}\right) \\
& \cdot \exp \left(-\frac{\sigma}{|\epsilon|^{r}} r_{b}(\underline{\beta})\left|h_{k_{0}} \cdots h_{1} \tau\right|\right) \\
& \cdot \frac{\exp \left(\left(\sigma /|\epsilon|^{r}\right) r_{b}(\underline{\beta})\left|h_{k_{0}} \cdots h_{1} \tau\right|\right)}{1+\left|h_{k_{0}} \cdots h_{1} \tau\right|^{2} /|\epsilon|^{2 r}} \\
& \cdot M_{k_{0}}\left(h_{1}, \ldots, h_{k_{0}-1}\right) d h_{k_{0}} \cdots d h_{1},
\end{aligned}
$$

where $M_{k_{0}}\left(h_{1}, \ldots, h_{k_{0}-1}\right)$ is a monic monomial for $k_{0} \geq 2$, while $M_{1}=1$, for all $\tau \in \Omega_{\beta}$. We deduce that

$$
\begin{gathered}
\left|\tau^{s_{1}} \partial_{\tau}^{-k_{0}} V_{\underline{\beta}}(\tau, \epsilon)\right|\left(1+\frac{|\tau|^{2}}{|\epsilon|^{2 r}}\right) \exp \left(-\frac{\sigma}{|\epsilon|^{r}} r_{b}(\underline{\tilde{\beta}})|\tau|\right) \\
\leq|\tau|^{s_{1}+k_{0}}\left\|V_{\underline{\beta}}(\tau, \epsilon)\right\|_{\underline{\beta}, \epsilon, \sigma, r, \Omega_{\underline{\beta}}}\left(1+\frac{|\tau|^{2}}{|\epsilon|^{2 r}}\right) \\
\cdot \exp \left(-\frac{\sigma}{|\epsilon|^{r}}\left(r_{b}(\underline{\widetilde{\beta}})-r_{b}(\underline{\beta})\right)|\tau|\right)
\end{gathered}
$$

for all $\tau \in \Omega_{\tilde{\beta}} \subset \Omega_{\underline{\beta}}$. By definition of $r_{b}$, one has

$$
r_{b}(\underline{\tilde{\beta}})-r_{b}(\underline{\beta})=\sum_{n=|\underline{\beta}|+1}^{|\widetilde{\beta}|} \frac{1}{(n+1)^{b}} \geq \frac{|\underline{\tilde{\beta}}|-|\underline{\beta}|}{(|\underline{\tilde{\beta}}|+1)^{b}} .
$$

Now, we recall the classical estimates: for all integers $m_{1}, m_{2} \geq$ 0 ,

$$
\sup _{x \geq 0} x^{m_{1}} e^{-m_{2} x}=\left(\frac{m_{1}}{m_{2}}\right)^{m_{1}} e^{-m_{1}}
$$

holds. Using (70), we deduce that

$$
\begin{gathered}
\left(|\tau|^{s_{1}+k_{0}}+\frac{|\tau|^{s_{1}+k_{0}+2}}{|\epsilon|^{2 r}}\right) \exp \left(-\frac{\sigma}{|\epsilon|^{r}}\left(\frac{|\underline{\tilde{\beta}}|-|\underline{\beta}|}{(|\underline{\tilde{\beta}}|+1)^{b}}\right)|\tau|\right) \\
\leq|\epsilon|^{r\left(s_{1}+k_{0}\right)}\left((|\underline{\tilde{\beta}}|+1)^{b\left(s_{1}+k_{0}\right)}\left(\frac{\left(s_{1}+k_{0}\right) e^{-1}}{\sigma(|\underline{\tilde{\beta}}|-|\underline{\beta}|)}\right)^{s_{1}+k_{0}}\right. \\
+(|\underline{\tilde{\beta}}|+1)^{b\left(s_{1}+k_{0}+2\right)} \\
\left.\cdot\left(\frac{\left(s_{1}+k_{0}+2\right) e^{-1}}{\sigma(|\widetilde{\beta}|-|\underline{\beta}|)}\right)^{s_{1}+k_{0}+2}\right) .
\end{gathered}
$$

Taking into account (68), (69), and (71), we get (66).

In the next proposition, we study the convolution product of functions in the spaces $E_{\underline{\beta}, \epsilon, \sigma, r, \Omega_{\beta}}$.

Proposition 10. Let $\beta^{1}=\left(\beta_{0}^{1}, \ldots, \beta_{l+1}^{1}\right) \in \mathbb{N}^{l+2}$ and $\beta^{2}=\left(\beta_{0}^{2}\right.$, $\left.\ldots, \beta_{l+1}^{2}\right) \in \mathbb{N}^{l+2}$. Let $\bar{\beta} \in \mathbb{N}^{l+2}$ such that $|\beta| \geq\left|\underline{\beta}^{1}\right|+\left|\overline{\beta^{2}}\right|$. Then, for any $V_{\underline{\beta}^{1}}(\tau, \epsilon) \in \bar{E}_{\underline{\beta}^{1}, \epsilon, \sigma, r, \Omega_{\beta^{1}}}, V_{\beta^{2}}(\tau, \bar{\epsilon}) \in \bar{E}_{\underline{\beta}^{2}, \epsilon, \sigma, r, \Omega_{\beta^{2}}}$, the convolution product $V_{\beta^{1}}(\tau, \epsilon) * V_{\beta^{2}}(\tau, \epsilon)$ belongs to $E_{\beta, \epsilon, \sigma, r, \Omega_{\beta^{\prime}}}$, for all $\epsilon \in D\left(0, \epsilon_{0}\right) \backslash\{0\}$. Moreover, there exists a universal constant $C_{5}>0$ such that

$$
\begin{aligned}
& \left\|\int_{0}^{\tau} V_{\underline{\beta}^{1}}(\tau-s, \epsilon) V_{\underline{\beta}^{2}}(s, \epsilon) d s\right\|_{\underline{\beta}, \epsilon, \sigma, r, \Omega_{\underline{\beta}}} \\
& \leq C_{5}|\epsilon|^{r}\left\|V_{\underline{\beta}^{1}}(\tau, \epsilon)\right\|_{\underline{\underline{\beta}}^{1}, \epsilon, \sigma, r, \Omega_{\underline{\beta}^{1}}}\left\|V_{\underline{\beta}^{2}}(\tau, \epsilon)\right\|_{\underline{\beta}^{2}, \epsilon, \sigma, r, \Omega_{\underline{\beta}^{2}}}
\end{aligned}
$$

for all $V_{\underline{\beta}^{1}}(\tau, \epsilon) \in E_{\underline{\beta}^{1}, \epsilon, \sigma, r, \Omega_{\beta^{1}}}, V_{\underline{\beta}^{2}}(\tau, \epsilon) \in E_{\underline{\beta}^{2}, \epsilon, \sigma, r, \Omega_{\beta^{2}}}$.

Proof. We mimic the proof of Lemma 3 in [17]. One can write

$$
\begin{aligned}
& \int_{0}^{\tau} V_{\underline{\beta}^{1}}(\tau-s, \epsilon) V_{\underline{\beta}^{2}}(s, \epsilon) d s \\
& =\int_{0}^{\tau} V_{\underline{\beta}^{1}}(\tau-s, \epsilon)\left(1+\frac{|\tau-s|^{2}}{|\epsilon|^{2 r}}\right) \\
& \cdot \exp \left(-\frac{\sigma}{|\epsilon|^{r}} r_{b}\left(\underline{\beta^{1}}\right)|\tau-s|\right) \\
& \cdot V_{\beta^{2}}(s, \epsilon)\left(1+\frac{|s|^{2}}{|\epsilon|^{2 r}}\right) \exp \left(-\frac{\sigma}{|\epsilon|^{r}} r_{b}\left(\underline{\beta^{2}}\right)|s|\right) \\
& \cdot \frac{\exp \left(\left(\sigma /|\epsilon|^{r}\right)\left(r_{b}\left(\underline{\beta^{1}}\right)|\tau-s|+r_{b}\left(\underline{\beta}^{2}\right)|s|\right)\right)}{\left(1+\left(|\tau-s|^{2} /|\epsilon|^{2 r}\right)\right)\left(1+\left(|s|^{2} /|\epsilon|^{2 r}\right)\right)} d s
\end{aligned}
$$


for all $\tau \in \Omega_{\beta} \subset \Omega_{\beta^{1}} \cap \Omega_{\beta^{2}}$. We deduce that

$$
\begin{aligned}
& \left|\int_{0}^{\tau} V_{\underline{\beta}^{1}}(\tau-s, \epsilon) V_{\underline{\beta}^{2}}(s, \epsilon) d s\right| \\
& \leq\left\|V_{\underline{\beta}^{1}}(\tau, \epsilon)\right\|_{\underline{\beta}^{1}, \epsilon, \sigma, r, \Omega_{\underline{\beta}^{1}}}\left\|V_{\underline{\beta}^{2}}(\tau, \epsilon)\right\|_{\underline{\beta}^{2}, \epsilon, \sigma, r, \Omega_{\underline{\beta}^{2}}} \\
& \quad \cdot \int_{0}^{1} \frac{|\tau| \exp \left(\sigma|\tau| /|\epsilon|^{r}\right)\left(r_{b}\left(\underline{\beta}^{1}\right)(1-h)+r_{b}\left(\underline{\beta}^{2}\right) h\right)}{\left(1+\left(|\tau|^{2} /|\epsilon|^{2 r}\right)(1-h)^{2}\right)\left(1+\left(|\tau|^{2} /|\epsilon|^{2 r}\right) h^{2}\right)} d h
\end{aligned}
$$

for all $\tau \in \Omega_{\beta}$. Since $r_{b}$ is increasing, one has

$$
r_{b}\left(\underline{\beta}^{1}\right)(1-h)+r_{b}\left(\underline{\beta}^{2}\right) h \leq r_{b}(\underline{\beta}) .
$$

Therefore,

$$
\begin{aligned}
& \left(1+\frac{|\tau|^{2}}{|\epsilon|^{2 r}}\right) \exp \left(-\frac{\sigma}{|\epsilon|^{r}} r_{b}(\underline{\beta})|\tau|\right) \\
& \quad \cdot \int_{0}^{1} \frac{|\tau| \exp \left(\sigma|\tau| /|\epsilon|^{r}\right)\left(r_{b}\left(\underline{\beta^{1}}\right)(1-h)+r_{b}\left(\underline{\beta^{2}}\right) h\right)}{\left(1+\left(|\tau|^{2} /|\epsilon|^{2 r}\right)(1-h)^{2}\right)\left(1+\left(|\tau|^{2} /|\epsilon|^{2 r}\right) h^{2}\right)} d h \\
& \leq \int_{0}^{1} \frac{\left(1+\left(|\tau|^{2} /|\epsilon|^{2 r}\right)\right)|\tau|}{\left(1+\left(|\tau|^{2} /|\epsilon|^{2 r}\right)(1-h)^{2}\right)\left(1+\left(|\tau|^{2} /|\epsilon|^{2 r}\right) h^{2}\right)} d h \\
& =J(|\tau|,|\epsilon|)
\end{aligned}
$$

for all $\tau \in \Omega_{\beta}$. Now, from [28], we know that there exists a universal constant $C_{5}>0$ such that

$$
\frac{J\left(|\epsilon|^{r}|\tau|,|\epsilon|\right)}{|\epsilon|^{r}} \leq C_{5}
$$

for all $\tau \in \mathbb{C}, \epsilon \in \mathbb{C}^{*}$. We deduce that

$$
\sup _{\tau \in \mathbb{C}} \frac{J(|\tau|,|\epsilon|)}{|\epsilon|^{r}}=\sup _{\tau \in \mathbb{C}} \frac{J\left(|\epsilon|^{r}|\tau|,|\epsilon|\right)}{|\epsilon|^{r}} \leq C_{5}
$$

for all $\epsilon \in \mathbb{C}^{*}$. Finally, gathering (74), (76), and (78) yields estimates (72).

Corollary 11. Let $l_{0} \geq 0$ be an integer. The operator $\partial_{\tau}^{-l_{0}}$ defines a bounded linear map from $E_{\beta, \epsilon, \sigma, r, \Omega_{\beta}}$ into itself, for all $\epsilon \in D\left(0, \epsilon_{0}\right) \backslash\{0\}$. Moreover, there exists a constant $C_{6}>0$ (depending on $l_{0}, \sigma$ ) such that

$$
\left\|\partial_{\tau}^{-l_{0}} V_{\underline{\beta}}(\tau, \epsilon)\right\|_{\underline{\beta}, \epsilon, \sigma, r, \Omega_{\underline{\beta}}} \leq C_{6}|\epsilon|^{r l_{0}}\left\|V_{\underline{\beta}}(\tau, \epsilon)\right\|_{\underline{\beta}, \epsilon, \sigma, r, \Omega_{\underline{\beta}}}
$$

for all $V_{\underline{\beta}}(\tau, \epsilon) \in E_{\underline{\beta}, \epsilon, \sigma, r, \Omega_{\underline{\beta}}}$.

Proof. We carry out a similar proof as in Corollary 1 from [17]. We denote by $\chi_{\mathbb{C}}$ the function equal to 1 on $\mathbb{C}$. By definition, we put $\chi_{\mathbb{C}}^{* 1}=\chi_{\mathbb{C}}$ and $\chi_{\mathbb{C}}^{* l}$ means the convolution product of $\chi_{\mathbb{C}}, l-1$ times for $l \geq 2$. By definition, we can write $\partial_{\tau}^{-l_{0}} V_{\beta}(\tau, \epsilon)=\left(\chi_{\mathbb{C}}(\tau)\right)^{* l_{0}} * V_{\beta}(\tau, \epsilon)$. From Proposition 10, there exists a (universal) constant $C_{5}>0$ such that

$$
\begin{aligned}
& \left\|\partial_{\tau}^{-l_{0}} V_{\underline{\beta}}(\tau, \epsilon)\right\|_{\left(\underline{\beta}, \epsilon, \sigma, r, \Omega_{\underline{\beta}}\right)} \\
& \quad \leq C_{5}^{l_{0}}|\epsilon|^{r l_{0}}\left\|\chi_{\mathbb{C}}(\tau)\right\|_{\left(\underline{0}, \epsilon, \sigma, r, \Omega_{\underline{0}}\right)}^{l_{0}}\left\|V_{\underline{\beta}}(\tau, \epsilon)\right\|_{\left(\underline{\beta}, \epsilon, \sigma, r, \Omega_{\underline{\beta}}\right)},
\end{aligned}
$$

where $\underline{0}=(0, \ldots, 0) \in \mathbb{N}^{l+2}$. By Definition 7 and using formula (70), we have that

$$
\begin{aligned}
& \left\|\chi_{\mathbb{C}}(\tau)\right\|_{\left(\underline{0}, \epsilon, \sigma, r, \Omega_{\underline{0}}\right)} \\
& =\sup _{\tau \in \Omega_{\underline{0}}}\left(1+\frac{|\tau|^{2}}{|\epsilon|^{2 r}}\right) \exp \left(-\frac{\sigma}{|\epsilon|^{r}}|\tau|\right) \leq 1+\left(\frac{2 e^{-1}}{\sigma}\right)^{2} .
\end{aligned}
$$

From estimates (80) and (81), we get inequality (79).

The next proposition involves bound estimates for multiplication operators of bounded holomorphic functions.

Proposition 12. Let $a(\tau, \epsilon)$ be a holomorphic function on $\Omega_{\underline{0} \times D\left(0, \epsilon_{0}\right) \backslash\{0\}}$ such that there exists a constant $M>0$ with $\sup _{(\tau, \epsilon) \in \Omega_{0 \times D\left(0, \epsilon_{0}\right) \backslash\{0\}}}|a(\tau, \epsilon)| \leq M$. Then, the multiplication by $a(\tau, \epsilon)$ is a bounded linear operator from $E_{\beta, \epsilon, \sigma, r, \Omega_{\beta}}$ into itself, for all $\epsilon \in D\left(0, \epsilon_{0}\right) \backslash\{0\}$. Moreover, the inequality

$$
\left\|a(\tau, \epsilon) V_{\underline{\beta}}(\tau, \epsilon)\right\|_{\underline{\beta}, \epsilon, \sigma, r, \Omega_{\underline{\beta}}} \leq M\left\|V_{\underline{\beta}}(\tau, \epsilon)\right\|_{\underline{\beta}, \epsilon, \sigma, r, \Omega_{\underline{\beta}}}
$$

holds for all $V_{\underline{\beta}}(\tau, \epsilon) \in E_{\underline{\beta}, \epsilon, \sigma, r, \Omega_{\underline{\beta}}}$.

Proof. The proof is a direct consequence of Definition 7 for the norm $\|\cdot\|_{\underline{\beta}, \epsilon, \sigma, r, \Omega_{\beta}}$.

2.3. A Global Cauchy Problem. We keep the same notations as in the previous section. In the following, we introduce some definitions. Let $\mathscr{A}_{1}$ be a finite subset of $\mathbb{N}^{3}$ and let $\mathscr{A}_{2}$ be a finite subset of $\mathbb{N}^{2}$. Let $l \geq 1$ be an integer.

For all $\left(k_{0}, k_{1}, k_{2}\right) \in \mathscr{A}_{1}$, we denote by $I_{\left(k_{0}, k_{1}, k_{2}\right)}$ a finite subset of $\mathbb{N}^{2}$. For all $\left(k_{0}, k_{1}, k_{2}\right) \in \mathscr{A}_{1}$, all $\left(s_{1}, s_{2}\right) \in$ $I_{\left(k_{0}, k_{1}, k_{2}\right)}$, and all integers $\beta_{0}, \beta_{l+1} \geq 0$, we denote by $a_{s_{1}, s_{2}, k_{0}, k_{1}, k_{2}, \beta_{0}, \beta_{l+1}}(\tau, \epsilon)$ some holomorphic function on $\Omega_{\underline{0}} \times$ $D\left(0, \epsilon_{0}\right)$ which satisfies the estimates: there exist constants $\rho, \rho^{\prime}>0, \mathfrak{a}_{s_{1}, s_{2}, k_{0}, k_{1}, k_{2}}>0$ with

$$
\begin{aligned}
& \sup _{(\tau, \epsilon) \in \Omega_{\underline{0}} \times D\left(0, \epsilon_{0}\right)}\left|a_{s_{1}, s_{2}, k_{0}, k_{1}, k_{2}, \beta_{0}, \beta_{l+1}}(\tau, \epsilon)\right| \\
& \leq \mathfrak{a}_{s_{1}, s_{2}, k_{0}, k_{1}, k_{2}}\left(\frac{e^{-\rho^{\prime}}}{2}\right)^{\beta_{0}}\left(\frac{1}{2 \rho}\right)^{\beta_{l+1}} \beta_{0} ! \beta_{l+1} !
\end{aligned}
$$


for all $\left(k_{0}, k_{1}, k_{2}\right) \in \mathscr{A}_{1}$, we consider the series

$$
\begin{aligned}
& a_{\left(k_{0}, k_{1}, k_{2}\right)}(\tau, z, x, \epsilon) \\
& =\sum_{\left(s_{1}, s_{2}\right) \in I_{\left(k_{0}, k_{1}, k_{2}\right)}} \sum_{\beta_{0} \geq 0, \beta_{l+1} \geq 0} a_{s_{1}, s_{2}, k_{0}, k_{1}, k_{2}, \beta_{0}, \beta_{l+1}} \\
& \cdot(\tau, \epsilon) \tau^{s_{1}} \epsilon^{-s_{2}} \frac{e^{i z \beta_{0}}}{\beta_{0} !} \frac{x^{\beta_{l+1}}}{\beta_{l+1} !}
\end{aligned}
$$

which define holomorphic functions on $\Omega_{\underline{0}} \times H_{\rho^{\prime}} \times D(0, \rho) \times$ $D\left(0, \epsilon_{0}\right) \backslash\{0\}$, where $H_{\rho^{\prime}}$ is defined as the following strip in $\mathbb{C}$ :

$$
H_{\rho^{\prime}}=\left\{z \in \frac{\mathbb{C}}{|\operatorname{Im}(z)|}<\rho^{\prime}\right\} .
$$

For all $\left(l_{0}, l_{1}\right) \in \mathscr{A}_{2}$, we denote by $J_{\left(l_{0}, l_{1}\right)}$ a finite subset of $\mathbb{N}$. For all $m_{1} \in J_{\left(l_{0}, l_{1}\right)}$ and all integers $\beta_{0}, \beta_{l+1} \geq 0$, we denote by $\alpha_{m_{1}, l_{0}, l_{1}, \beta_{0}, \beta_{l+1}}(\tau, \epsilon)$ some bounded holomorphic function on $\Omega_{0} \times D\left(0, \epsilon_{0}\right)$ with the following estimates: there exists a constant $\mathfrak{a}_{m_{1}, l_{0}, l_{1}}>0$ such that

$$
\begin{aligned}
& \sup _{(\tau, \epsilon) \in \Omega_{\underline{0}} \times D\left(0, \epsilon_{0}\right)}\left|\alpha_{m_{1}, l_{0}, l_{1}, \beta_{0}, \beta_{l+1}}(\tau, \epsilon)\right| \\
& \leq \mathfrak{a}_{m_{1}, l_{0}, l_{1}}\left(\frac{e^{-\rho^{\prime}}}{2}\right)^{\beta_{0}}\left(\frac{1}{2 \rho}\right)^{\beta_{l+1}} \beta_{0} ! \beta_{l+1} !
\end{aligned}
$$

for all $\beta_{0}, \beta_{l+1} \geq 0$.

For all $\left(l_{0}, l_{1}\right) \in \mathscr{A}_{2}$, we consider the series

$$
\begin{aligned}
& \alpha_{\left(l_{0}, l_{1}\right)}(\tau, z, x, \epsilon) \\
& =\sum_{m_{1} \in J_{\left(l_{0}, l_{1}\right)}} \sum_{\beta_{0} \geq 0, \beta_{l+1} \geq 0} \alpha_{m_{1}, l_{0}, l_{1}, \beta_{0}, \beta_{l+1}}(\tau, \epsilon) \epsilon^{-m_{1}} \frac{e^{i z \beta_{0}}}{\beta_{0} !} \frac{x^{\beta_{l+1}}}{\beta_{l+1} !}
\end{aligned}
$$

which define holomorphic functions on $\Omega_{0} \times H_{\rho^{\prime}} \times D(0, \rho) \times$ $D\left(0, \epsilon_{0}\right) \backslash\{0\}$.

Let $l \geq 1$ be an integer and let $\xi_{0}=1$ and $\xi_{1}, \ldots, \xi_{l}$ be real algebraic numbers such that the family $\left\{1, \xi_{1}, \ldots, \xi_{l}\right\}$ is $\mathbb{Z}$ linearly independent (this means that each $\xi_{j}$ is a real root of a polynomial $P_{j} \in \mathbb{Z}[X]$ and if there exist integers $k_{0}, \ldots, k_{l} \in$ $\mathbb{Z}$ such that $k_{0}+\sum_{j=1}^{l} k_{j} \xi_{j}=0$, then $k_{j}=0$ for $0 \leq j \leq$ l). Due to the classical primitive element theorem of Artin, we consider an algebraic number field $\mathbb{K}=\mathbb{Q}(\xi)$ containing the numbers $\xi_{j}, 1 \leq j \leq l$ and we denote by $h+1 \geq 1$ its degree (that is the dimension of the vector space $\mathbb{Q}(\xi)$ over $\mathbb{Q})$. The following lemma is a direct consequence of Theorem 11 in [29].

Lemma 13. There exists a constant $C_{\xi_{1}, \ldots, \xi_{l}}>0$ (depending on $\left.\xi_{1}, \ldots, \xi_{l}\right)$ such that, for any $\underline{k}=\left(k_{0}, \ldots, k_{l}\right) \in \mathbb{Z}^{l+1} \backslash\{0\}$, the inequality

$$
\begin{aligned}
\mid k_{0} & +k_{1} \xi_{1}+\cdots+k_{l} \xi_{l} \mid \\
& \geq \frac{C_{\xi_{1}, \ldots, \xi_{l}}}{\left(\max _{j=0}^{l}\left|k_{j}\right|\right)^{h}} \geq \frac{C_{\xi_{1}, \ldots, \xi_{l}}}{\left(\left|k_{0}\right|+\cdots+\left|k_{l}\right|\right)^{h}}
\end{aligned}
$$

holds.
Example 14. Let $\xi$ be an algebraic number. Assume that the degree of $\mathbb{Q}(\xi)$ is $h+1$. Then, the algebraic numbers $\left\{1, \xi, \ldots, \xi^{h}\right\}$ are $\mathbb{Z}$-linearly independent and inequality (88) above holds for $\xi_{j}=\xi^{j}, 0 \leq j \leq h$. In that case, one recovers Lemma 2.1 of [6].

Let $S, r_{1}, r_{2} \geq 1$ be integers. We put

$$
\rho_{\underline{\beta}}=\frac{C_{\xi_{1}, \ldots, \xi_{l}}^{r_{1} / r_{2}}}{\left(2\left(\beta_{0}+1+\beta_{1}+\cdots+\beta_{l+1}\right)^{h r_{1} / r_{2}}\right)}
$$

for all $\beta=\left(\beta_{0}, \ldots, \beta_{l+1}\right) \in \mathbb{N}^{l+2}$. We consider an unbounded sector $\bar{S}_{d} \subset \mathbb{C}$ centered at 0 such that

$$
\arg (\tau) \neq \frac{2 k+1}{r_{2}} \pi, \quad 0 \leq k \leq r_{2}-1,
$$

for all $\tau \in S_{d}$. As in the previous section, we put $\Omega_{\beta}=$ $D\left(0, \rho_{\beta}\right) \cup S_{d}$. For all $0 \leq j \leq S-1$, we choose a set of functions $V_{\left(\beta_{0}, \ldots, \beta_{l}, j\right)}(\tau, \epsilon) \in E_{\left(\beta_{0}, \ldots, \beta_{l}, j\right), \epsilon, \sigma, r, \Omega_{\left(\beta_{0}, \ldots, \beta_{l}, j\right)}}$ for all $\beta_{0}, \ldots, \beta_{l} \geq 0$ and we consider the formal series

$$
\begin{aligned}
V_{j}(\tau, z, \epsilon) \\
\quad=\sum_{\beta_{0}, \ldots, \beta_{l} \geq 0} V_{\left(\beta_{0}, \ldots, \beta_{l}, j\right)}(\tau, \epsilon) \frac{\exp \left(i z\left(\sum_{j=0}^{l} \beta_{j} \xi_{j}\right)\right)}{\beta_{0} ! \cdots \beta_{l} !}
\end{aligned}
$$

for all $0 \leq j \leq S-1$.

We consider the following Cauchy problem:

$$
\begin{aligned}
& \left(\tau^{r_{2}}+\left(-i \partial_{z}+1\right)^{r_{1}}\right) \partial_{x}^{S} V(\tau, z, x, \epsilon) \\
& =\sum_{\left(k_{0}, k_{1}, k_{2}\right) \in \mathscr{A}_{1}} a_{\left(k_{0}, k_{1}, k_{2}\right)}(\tau, z, x, \epsilon) \partial_{\tau}^{-k_{0}} \partial_{z}^{k_{1}} \partial_{x}^{k_{2}} V(\tau, z, x, \epsilon) \\
& \quad+\sum_{\left(l_{0}, l_{1}\right) \in \mathscr{A}_{2}, l_{1} \geq 2} \alpha_{\left(l_{0}, l_{1}\right)}(\tau, z, x, \epsilon) \partial_{\tau}^{-l_{0}}(V(\tau, z, x, \epsilon))^{* l_{1}},
\end{aligned}
$$

where $V^{* 1}=V$ and $V^{* l_{1}}, l_{1} \geq 2$, stands for the convolution product of $V$ applied $l_{1}-1$ times with respect to $\tau$, for given initial conditions

$$
\left(\partial_{z}^{j} V\right)(\tau, z, 0, \epsilon)=V_{j}(\tau, z, \epsilon), \quad 0 \leq j \leq S-1 .
$$

In the sequel, we will need the next lemma.

Lemma 15. There exists a constant $C_{7}>0$ (depending on $\left.r_{1}, r_{2}, C_{\xi_{1}, \ldots, \xi_{l}}, S_{d}\right)$ such that

$$
\left|\frac{1}{\tau^{r_{2}}+\left(1+\sum_{j=0}^{l} \beta_{j} \xi_{j}\right)^{r_{1}}}\right| \leq C_{7}\left(1+\sum_{j=0}^{l} \beta_{j}\right)^{h r_{1}}
$$

for all $\tau \in \Omega_{\left(\beta_{0}, \ldots, \beta_{l}, \beta_{l+1}+S\right)}$, for all $\beta_{j} \geq 0,0 \leq j \leq l+1$.

Proof. We put $A=1+\sum_{j=0}^{l} \beta_{j} \xi_{j}$. The following partial fraction decomposition holds:

$$
\frac{1}{\tau^{r_{2}}+A^{r_{1}}}=\sum_{k=0}^{r_{2}-1} \frac{A_{k}}{\tau-|A|^{r_{1} / r_{2}} e^{i \pi\left((2 k+1) / r_{2}\right)}},
$$


where

$$
A_{k}=\frac{1}{r_{2}} \frac{e^{-i \pi\left((2 k+1)\left(r_{2}-1\right) / r_{2}\right)}}{|A|^{r_{1}-\left(r_{1} / r_{2}\right)}}
$$

for all $0 \leq k \leq r_{2}-1$. Now, there exists some constant $C_{8}>0$ (depending on $S_{d}$ ) such that

$$
|\tau-| 1+\left.\sum_{j=0}^{l} \beta_{j} \xi_{j}\right|^{r_{1} / r_{2}} e^{i \pi\left((2 k+1) / r_{2}\right)}\left|\geq C_{8}\right| 1+\left.\sum_{j=0}^{l} \beta_{j} \xi_{j}\right|^{r_{1} / r_{2}}
$$

for all $\tau \in \Omega_{\left(\beta_{0}, \ldots, \beta_{l}, \beta_{l+1}+S\right)}$. Indeed, from (88), we know that

$$
\begin{aligned}
\left|1+\sum_{j=0}^{l} \beta_{j} \xi_{j}\right|^{r_{1} / r_{2}} & \geq \frac{C_{\xi_{1}, \ldots, \xi_{l}}^{r_{1} / r_{2}}}{\left(1+\sum_{j=0}^{l} \beta_{j}\right)^{h r_{1} / r_{2}}} \\
& >\frac{C_{\xi_{1}, \ldots, \xi_{l}}^{r_{1} / r_{2}}}{\left(1+\sum_{j=0}^{l+1} \beta_{j}+S\right)^{h r_{1} / r_{2}}}
\end{aligned}
$$

for all $\beta_{j} \geq 0,0 \leq j \leq l+1$. Let $\tau \in D\left(0, \rho_{\left(\beta_{0}, \ldots, \beta_{l}, \beta_{l+1}+S\right)}\right)$. From (98), we can write

$$
\tau=\frac{h}{2} e^{i \theta}\left|1+\sum_{j=0}^{l} \beta_{j} \xi_{j}\right|^{r_{1} / r_{2}}
$$

for some $0 \leq h<1$ and $\theta \in[0,2 \pi)$. Therefore,

$$
\begin{aligned}
\mid \tau & -\left|1+\sum_{j=0}^{l} \beta_{j} \xi_{j}\right|^{r_{1} / r_{2}} e^{i \pi\left((2 k+1) / r_{2}\right)} \mid \\
& =\left|1+\sum_{j=0}^{l} \beta_{j} \xi_{j}\right|^{r_{1} / r_{2}}\left|\frac{h}{2} e^{i \theta}-e^{i \pi\left((2 k+1) / r_{2}\right)}\right| \\
& \geq \frac{1}{2}\left|1+\sum_{j=0}^{l} \beta_{j} \xi_{j}\right|^{r_{1} / r_{2}} .
\end{aligned}
$$

Now, let $\tau \in S_{d}$. For all $k \in\left\{0, \ldots, r_{2}-1\right\}$, we can write

$$
\tau=s\left|1+\sum_{j=0}^{l} \beta_{j} \xi_{j}\right|^{r_{1} / r_{2}} e^{i\left(\pi\left((2 k+1) / r_{2}\right)+s_{k}\right)}
$$

where $s \geq 0$ and $s_{k} \in \mathbb{R}$. By construction of $S_{d}$, we have that $e^{i s_{k}} \neq 1$ for all $k \in\left\{0, \ldots, r_{2}-1\right\}$. As a result, there exists a constant $C_{9}>0$ (depending on $S_{d}$ ) with $\left|s e^{i s_{k}}-1\right| \geq C_{9}$, for all $s \geq 0$. Hence,

$$
\begin{aligned}
\mid \tau & -\left|1+\sum_{j=0}^{l} \beta_{j} \xi_{j}\right|^{r_{1} / r_{2}} e^{i \pi\left((2 k+1) / r_{2}\right)} \mid \\
& =\left|1+\sum_{j=0}^{l} \beta_{j} \xi_{j}\right|^{r_{1} / r_{2}}\left|s e^{i\left(\pi\left((2 k+1) / r_{2}\right)+s_{k}\right)}-e^{i \pi\left((2 k+1) / r_{2}\right)}\right| \\
& \geq C_{9}\left|1+\sum_{j=0}^{l} \beta_{j} \xi_{j}\right|^{r_{1} / r_{2}} .
\end{aligned}
$$

As a consequence, we get that (97) holds. On the other hand, from (88), we get that

$$
\frac{1}{|A|^{r_{1}}} \leq \frac{\left(1+\sum_{j=0}^{l} \beta_{j}\right)^{h r_{1}}}{C_{\xi_{1}, \ldots, \xi_{l}}^{r_{1}}}
$$

for all $\beta_{j} \geq 0,0 \leq j \leq l$. Gathering (95), (97), and (103), we deduce that

$$
\begin{aligned}
\frac{\left|A_{k}\right|}{\left.|\tau-| A\right|^{r_{1} / r_{2}} e^{i \pi\left((2 k+1) / r_{2}\right) \mid}} & \leq \frac{1}{r_{2} C_{8}|A|^{r_{1}}} \\
& \leq \frac{1}{r_{2} C_{8} C_{\xi_{1}, \ldots, \xi_{l}}^{r_{1}}}\left(1+\sum_{j=0}^{l} \beta_{j}\right)^{h r_{1}}
\end{aligned}
$$

for all $0 \leq k \leq r_{2}-1$. The lemma follows.

In the next proposition, we construct formal series solutions of (92) and (93).

Proposition 16. Under the assumption that

$$
S>k_{2}
$$

for all $\left(k_{0}, k_{1}, k_{2}\right) \in \mathscr{A}_{1}$, there exists a formal series

$$
\begin{aligned}
& V(\tau, z, x, \epsilon) \\
& =\sum_{\underline{\beta}=\left(\beta_{0}, \ldots, \beta_{l+1}\right) \in \mathbb{N}^{l+2}} V_{\underline{\beta}}(\tau, \epsilon) \frac{\exp \left(i z\left(\sum_{j=0}^{l} \beta_{j} \xi_{j}\right)\right)}{\beta_{0} ! \cdots \beta_{l} !} \frac{x^{\beta_{l+1}}}{\beta_{l+1} !}
\end{aligned}
$$

solution of (92) and (93), where the coefficients $\tau \mapsto V_{\beta}(\tau, \epsilon)$ belong to the space $\mathcal{O}\left(\Omega_{\beta}\right)$ for all $\underline{\beta} \in \mathbb{N}^{l+2}$ and satisfy the following recursion formula:

$$
\begin{aligned}
& \left(\tau^{r_{2}}+\left(1+\sum_{j=0}^{l} \beta_{j} \xi_{j}\right)^{r_{1}}\right) \frac{V_{\left(\beta_{0}, \ldots, \beta_{l}, \beta_{l+1}+S\right)}(\tau, \epsilon)}{\beta_{0} ! \cdots \beta_{l} ! \beta_{l+1} !} \\
& =\sum_{\left(k_{0}, k_{1}, k_{2}\right) \in \mathscr{A}_{1}\left(s_{1}, s_{2}\right) \in I_{\left(k_{0}, k_{1}, k_{2}\right)}} \sum_{\substack{\beta_{0}^{1}+\beta_{0}^{2}=\beta_{0} \\
\beta_{l+1}^{1}+\beta_{l+1}^{2}=\beta_{l+1}}} \frac{a_{s_{1}, s_{2}, k_{0}, k_{1}, k_{2}, \beta_{0}^{1}, \beta_{l+1}^{1}}(\tau, \epsilon)}{\beta_{0}^{1} ! \beta_{l+1}^{1} !} \tau^{s_{1}} \epsilon^{-s_{2}} \frac{\partial_{\tau}^{-k_{0}}\left(V_{\left(\beta_{0}^{2}, \beta_{1}, \ldots, \beta_{l}, \beta_{l+1}^{2}+k_{2}\right)}(\tau, \epsilon)\right)}{\beta_{0}^{2} ! \beta_{1} ! \cdots \beta_{l} ! \beta_{l+1}^{2} !} i^{k_{1}}\left(\beta_{0}^{2}+\sum_{j=1}^{l} \beta_{j} \xi_{j}\right)^{k_{1}}
\end{aligned}
$$




$$
\begin{aligned}
& +\sum_{\left(l_{0}, l_{1}\right) \in \mathscr{A}_{2}, l_{1} \geq 2} \sum_{m \in J_{\left(l_{0}, l_{1}\right)}} \sum_{\substack{\beta_{0}^{-1}+\beta_{0}^{0}+\cdots+\beta_{0}^{l_{1}-1}=\beta_{0} \\
\beta_{j}^{0}+\cdots+\beta_{j}^{l_{1}-1}=\beta_{j}, 1 \leq j \leq l \\
\beta_{l+1}^{-1}+\beta_{l+1}^{0}+\cdots+\beta_{l+1}^{l_{1}-1}=\beta_{l+1}}} \frac{\alpha_{m_{1}, l_{0}, l_{1}, \beta_{0}^{-1}, \beta_{l+1}^{-1}}(\tau, \epsilon) \epsilon^{-m_{1}}}{\beta_{0}^{-1} ! \beta_{l+1}^{-1} !} \\
& \times \frac{\partial_{\tau}^{-l_{0}}\left(V_{\left(\beta_{0}^{0}, \ldots, \beta_{l+1}^{0}\right)}(\tau, \epsilon) * \cdots * V_{\left(\beta_{0}^{l_{1}-1}, \ldots, \beta_{l+1}^{l_{1}-1}\right)}(\tau, \epsilon)\right)}{\Pi_{m=0}^{l_{1}-1} \Pi_{j=0}^{l+1} \beta_{j}^{m !}}
\end{aligned}
$$

for all $\beta_{0}, \ldots, \beta_{l+1} \geq 0$, all $\tau \in \Omega_{\left(\beta_{0}, \ldots, \beta_{l}, \beta_{l+1}+S\right)}$, and all $\epsilon \epsilon$ $D\left(0, \epsilon_{0}\right) \backslash\{0\}$.

Proof. By hypothesis, we know in particular that $V_{\left(\beta_{0}, \ldots, \beta_{l}, j\right)}(\tau, \epsilon)$ belongs to $\mathcal{O}\left(\Omega_{\left(\beta_{0}, \ldots, \beta_{l}, j\right)}\right)$ for any $\beta_{0}, \ldots, \beta_{l} \geq 0$, all $0 \leq j \leq S-1$, and all $\epsilon \in D\left(0, \epsilon_{0}\right) \backslash\{0\}$. Since $\Omega_{\underline{\beta}^{\prime}} \subset \Omega_{\underline{\beta}}$ when $\left|\beta^{\prime}\right|>|\beta|$ and using Lemma 15, one gets that the functions $\tau \mapsto V_{\beta}(\tau, \epsilon)$ which are defined by recursion (107) actually belong to $\mathcal{O}\left(\Omega_{\beta}\right)$ for any $\beta \in \mathbb{N}^{l+2}$, for all $\epsilon \in D\left(0, \epsilon_{0}\right) \backslash\{0\}$. Direct computation by identification of the powers of $e^{i z}, \ldots, e^{i z \xi_{l}}$ and the powers of $x$ shows that the formal series (106) is solution of (92) and (93), if its coefficients $V_{\beta}(\tau, \epsilon)$ satisfy recursion (107).

In the next proposition, we state norm inequalities for the sequence $V_{\beta}$.

Proposition 17. We consider the sequence offunctions $V_{\beta}(\tau, \epsilon)$ defined by recursion (107) for given initial data $V_{\left(\beta_{0}, \ldots, \beta_{l}, j\right)}(\tau, \epsilon)$ defined above for all $\beta_{0}, \ldots, \beta_{l} \geq 0,0 \leq j \leq S-1$. Then, for all $\underline{\beta} \in \mathbb{N}^{l+2}$ and all $\epsilon \in D\left(0, \epsilon_{0}\right) \backslash\{0\}$, the function $\tau \mapsto V_{\beta}(\tau, \epsilon)$ belongs to $E_{\underline{\beta}, \epsilon, \sigma, r, \Omega_{\underline{\beta}}}$. We put $w_{\underline{\beta}}(\epsilon)=\left\|V_{\underline{\beta}}(\tau, \epsilon)\right\|_{\underline{\beta}, \epsilon, \sigma, r, \Omega_{\underline{\beta}}}$, for all $\beta \in \mathbb{N}^{l+2}$ and all $\epsilon \in D\left(0, \epsilon_{0}\right) \backslash\{0\}$. Then, the sequence $w_{\beta}(\epsilon)$ satisfies the following estimates. There exist constants $C_{10}>0$ (depending on $r_{1}, r_{2}, C_{\xi_{1}, \ldots, \xi_{l}}, S_{d}$ ) and $C_{11}>0$ (depending on $\sigma)$ such that

$$
\begin{aligned}
& \frac{w_{\left(\beta_{0}, \ldots, \beta_{l}, \beta_{l+1}+S\right)}(\epsilon)}{\beta_{0} ! \cdots \beta_{l} ! \beta_{l+1} !} \\
& \leq \sum_{\left(k_{0}, k_{1}, k_{2}\right) \in \mathscr{A}_{1}} \sum_{\left(s_{1}, s_{2}\right) \in I_{\left(k_{0}, k_{1}, k_{2}\right)}} C_{10}\left(1+\sum_{j=0}^{l} \beta_{j}\right)^{h r_{1}} \\
& \sum_{\substack{\beta_{0}^{1}+\beta_{0}^{2}=\beta_{0} \\
\beta_{l+1}^{1}+\beta_{l+1}^{2}=\beta_{l+1}}} \frac{A_{s_{1}, s_{2}, k_{0}, k_{1}, k_{2}, \beta_{0}^{1}, \beta_{l+1}^{1}}}{\beta_{0}^{1} ! \beta_{l+1}^{1} !} \\
& \cdot \frac{w_{\left(\beta_{0}^{2}, \beta_{1}, \ldots, \beta_{l}, \beta_{l+1}^{2}+k_{2}\right)}(\epsilon)}{\beta_{0}^{2} ! \beta_{1} ! \cdots \beta_{l} ! \beta_{l+1}^{2} !}|\epsilon|^{r\left(s_{1}+k_{0}\right)-s_{2}} \\
& \cdot\left(\left(\sum_{j=0}^{l+1} \beta_{j}+S\right)^{b\left(s_{1}+k_{0}\right)}\left(\frac{\left(s_{1}+k_{0}\right) e^{-1}}{\sigma\left(S-k_{2}\right)}\right)^{s_{1}+k_{0}}\right.
\end{aligned}
$$

$$
\begin{gathered}
\cdot\left(\beta_{0}+\sum_{j=1}^{l} \beta_{j}\left|\xi_{j}\right|\right)^{k_{1}} \\
+\sum_{\substack{\left(l_{0}, l_{1}\right) \in \mathscr{A}_{2}, l_{1} \geq 2 \\
m_{1} \in J_{\left(l_{0}, l_{1}\right)}}} C_{10}\left(1+\sum_{j=0}^{l} \beta_{j}\right)^{h r_{1}} \\
\sum_{\substack{\beta_{0}^{-1}+\beta_{0}^{0}+\cdots+\beta_{0}^{l_{1}-1}=\beta_{0} \\
\beta_{j}^{0}+\cdots+\beta_{j}^{l_{1}-1}=\beta_{j}, 1 \leq j \leq l \\
\beta_{l+1}^{-1}+\beta_{l+1}^{0}+\cdots+\beta_{l+1}^{l_{1}-1}=\beta_{l+1}}} \frac{B_{m_{1}, l_{0}, l_{1}, \beta_{0}^{-1}, \beta_{l+1}^{-1}}}{\beta_{0}^{-1} ! \beta_{l+1}^{-1} !}
\end{gathered}
$$$$
\left.+\left(\sum_{j=0}^{l+1} \beta_{j}+S\right)^{b\left(s_{1}+k_{0}+2\right)}\left(\frac{\left(s_{1}+k_{0}+2\right) e^{-1}}{\sigma\left(S-k_{2}\right)}\right)^{s_{1}+k_{0}+2}\right)
$$$$
\cdot C_{11}^{l_{1}}|\epsilon|^{r\left(l_{0}+l_{1}-1\right)-m_{1}} \frac{\prod_{j=0}^{l_{1}-1} w_{\left(\beta_{0}^{j}, \ldots, \beta_{l+1}^{j}\right)}(\epsilon)}{\prod_{j=0}^{l_{1}-1} \beta_{0}^{j} ! \cdots \beta_{l+1}^{j} !}
$$

for all $\beta_{j} \geq 0,0 \leq j \leq l+1$, and all $\epsilon \in D\left(0, \epsilon_{0}\right) \backslash\{0\}$, where

$$
\begin{aligned}
& A_{s_{1}, s_{2}, k_{0}, k_{1}, k_{2}, \beta_{0}^{1}, \beta_{l+1}^{1}} \\
& \quad=\sup _{(\tau, \epsilon) \in \Omega_{\underline{0}} \times D\left(0, \epsilon_{0}\right) \backslash\{0\}}\left|a_{s_{1}, s_{2}, k_{0}, k_{1}, k_{2}, \beta_{0}^{1}, \beta_{l+1}^{1}}(\tau, \epsilon)\right|, \\
& B_{m_{1}, l_{0}, l_{1}, \beta_{0}^{-1}, \beta_{l+1}^{-1}} \\
& \quad \sup _{(\tau, \epsilon) \in \Omega_{\underline{0}} \times D\left(0, \epsilon_{0}\right) \backslash\{0\}}\left|\alpha_{m_{1}, l_{0}, l_{1}, \beta_{0}^{-1}, \beta_{l+1}^{-1}}(\tau, \epsilon)\right| .
\end{aligned}
$$

Proof. We apply the norm $\|\cdot\|_{\left(\beta_{0}, \ldots, \beta_{l}, \beta_{l+1}+S\right), \epsilon, \sigma, r, \Omega_{\left(\beta_{0}, \ldots, \beta_{l}, \beta_{l+1}+S\right)}}$ on the left and right hand side of equality (107) and use Propositions 9, 10, and 12, Lemma 15, and Corollary 11 in order to majorize the right hand side. Indeed, using Propositions 9, 10 , and 12, Corollary 11, Lemma 15 , and the estimates

$$
\left(\beta_{0}^{2}+\sum_{j=1}^{l} \beta_{j}\left|\xi_{j}\right|\right)^{k_{1}} \leq\left(\beta_{0}+\sum_{j=1}^{l} \beta_{j}\left|\xi_{j}\right|\right)^{k_{1}}
$$


we get a constant $C_{7}>0$ (depending on $r_{1}, r_{2}, C_{\xi_{1}, \ldots, \xi_{l}}, S_{d}$ ) such that

$$
\begin{aligned}
& \| \frac{1}{\tau^{r_{2}}+\left(1+\sum_{j=0}^{l} \beta_{j} \xi_{j}\right)^{r_{1}}} \frac{a_{s_{1}, s_{2}, k_{0}, k_{1}, k_{2}, \beta_{0}^{1}, \beta_{l+1}^{1}}(\tau, \epsilon)}{\beta_{0}^{1} ! \beta_{l+1}^{1} !} \\
& \cdot \tau^{s_{1}} \epsilon^{-s_{2}} \times \frac{\partial_{\tau}^{-k_{0}}\left(V_{\beta_{0}^{2}, \beta_{1}, \ldots, \beta_{l}, \beta_{l+1}^{2}+k_{2}}\right)(\tau, \epsilon)}{\beta_{0}^{2} ! \beta_{1} ! \cdots \beta_{l} ! \beta_{l+1}^{2} !} \\
& \cdot i^{k_{1}}\left(\beta_{0}^{2}+\sum_{j=1}^{l} \beta_{j} \xi_{j}\right)^{k_{1}} \| \\
& \leq C_{7}\left(1+\sum_{j=0}^{l} \beta_{j}\right)^{h r_{1}} \times \frac{A_{s_{1}, s_{2}, k_{0}, k_{1}, k_{2}, \beta_{0}^{1}, \beta_{l+1}^{1}}}{\beta_{0}^{1} ! \beta_{l+1}^{1} !} ! \\
& \cdot \frac{w_{\left(\beta_{0}^{2}, \beta_{1}, \ldots, \beta_{l}, \beta_{l+1}^{2}+k_{2}\right)}(\epsilon)}{\beta_{0}^{2} ! \beta_{1} ! \cdots \beta_{l} ! \beta_{l+1}^{2} !}|\epsilon|^{r\left(s_{1}+k_{0}\right)-s_{2}} \\
& \cdot\left(\left(\sum_{j=0}^{l+1} \beta_{j}+S\right)^{b\left(s_{1}+k_{0}\right)}\left(\frac{\left(s_{1}+k_{0}\right) e^{-1}}{\sigma\left(S-k_{2}\right)}\right)^{s_{1}+k_{0}}\right. \\
& \left.\quad+\left(\sum_{j=0}^{l+1} \beta_{j}+S\right)^{b\left(s_{1}+k_{0}+2\right)}\left(\frac{\left(s_{1}+k_{0}+2\right) e^{-1}}{\sigma\left(S-k_{2}\right)}\right)^{s_{1}+k_{0}+2}\right) \\
& \cdot\left(\beta_{0}+\sum_{j=1}^{l} \beta_{j}\left|\xi_{j}\right|\right)^{k_{1}}
\end{aligned}
$$

and using Propositions 10 and 12 and Corollary 11, we obtain a universal constant $C_{5}>0$ and some constants $C_{6}>0$ (depending on $l_{0}, \sigma$ ) and $C_{7}>0$ (depending on $\left.r_{1}, r_{2}, C_{\xi_{1}, \ldots, \xi_{l}}, S_{d}\right)$ such that

$$
\begin{gathered}
\| \frac{1}{\tau^{r_{2}}+\left(1+\sum_{j=0}^{l} \beta_{j} \xi_{j}\right)^{r_{1}}} \frac{\alpha_{m_{1}, l_{0}, l_{1}, \beta_{0}^{-1}, \beta_{l+1}^{-1}}(\tau, \epsilon) \epsilon^{-m_{1}}}{\beta_{0}^{-1} ! \beta_{l+1}^{-1} !} \\
\cdot \frac{\partial_{\tau}^{-l_{0}}\left(V_{\beta_{0}^{0}, \ldots, \beta_{l+1}^{0}}(\tau, \epsilon) * \cdots * V_{\beta_{0}^{l_{1}-1}, \ldots, \beta_{l+1}^{l_{1}-1}}(\tau, \epsilon)\right)}{\prod_{m=0}^{l_{1}-1} \Pi_{j=0}^{l+1} \beta_{j}^{m !} !} \|_{\left(\beta_{0}, \ldots, \beta_{l}, \beta_{l+1}+S\right)} \\
\leq C_{7}\left(1+\sum_{j=0}^{l} \beta_{j}\right)^{h r_{1}} \frac{B_{m_{1}, l_{0}, l_{1}, \beta_{0}^{-1}, \beta_{l+1}^{-1}}}{\beta_{0}^{-1} ! \beta_{l+1}^{-1} !} \\
\cdot C_{6} C_{5}^{l_{1}-1}|\epsilon|^{r\left(l_{0}+l_{1}-1\right)-m_{1}} \frac{\prod_{j=0}^{l_{1}-1} w_{\left(\beta_{0}^{j}, \ldots, \beta_{l+1}^{j}\right)}^{j}(\epsilon)}{\prod_{j=0}^{l_{1}-1} \beta_{0}^{j} ! \cdots \beta_{l+1}^{j} !} .
\end{gathered}
$$

We define the following formal series:

$$
\begin{aligned}
& \mathbb{A}_{s_{1}, s_{2}, k_{0}, k_{1}, k_{2}}\left(Z_{0}, X\right) \\
& \quad=\sum_{\beta_{0}, \beta_{l+1} \geq 0} A_{s_{1}, s_{2}, k_{0}, k_{1}, k_{2}, \beta_{0}, \beta_{l+1}} \frac{Z_{0}^{\beta_{0}}}{\beta_{0} !} \frac{X^{\beta_{l+1}}}{\beta_{l+1} !}, \\
& \mathbb{B}_{m_{1}, l_{0}, l_{1}}\left(Z_{0}, X\right) \\
& \quad=\sum_{\beta_{0}, \beta_{l+1} \geq 0} B_{m_{1}, l_{0}, l_{1}, \beta_{0}, \beta_{l+1}} \frac{Z_{0}^{\beta_{0}}}{\beta_{0} !} \frac{X^{\beta_{l+1}}}{\beta_{l+1} !} .
\end{aligned}
$$

We consider the following Cauchy problem:

$$
\begin{aligned}
& \partial_{X}^{S} U\left(Z_{0}, \ldots, Z_{l}, X, \epsilon\right) \\
& =\sum_{\left(k_{0}, k_{1}, k_{2}\right) \in \mathscr{A}_{1}} \sum_{\left(s_{1}, s_{2}\right) \in I_{\left(k_{0}, k_{1}, k_{2}\right)}} C_{10}\left(1+\sum_{j=0}^{l} Z_{j} \partial_{Z_{j}}\right)^{h r_{1}} \\
& \cdot\left(\left(\frac{\left(s_{1}+k_{0}\right) e^{-1}}{\sigma\left(S-k_{2}\right)}\right)^{s_{1}+k_{0}}\left(\sum_{j=0}^{l} Z_{j} \partial_{Z_{j}}+X \partial_{X}+S\right)^{b\left(s_{1}+k_{0}\right)}\right. \\
& \left.\quad+\left(\frac{\left(s_{1}+k_{0}+2\right) e^{-1}}{\sigma\left(S-k_{2}\right)}\right)^{s_{1}+k_{0}+2}\right) \\
& \left.\quad \cdot\left(\sum_{j=0}^{l} Z_{j} \partial_{Z_{j}}+X \partial_{X}+S\right)^{b\left(s_{1}+k_{0}+2\right)}\right) \\
& \quad \cdot\left(\sum_{j=0}^{l}\left|\xi_{j}\right| Z_{j} \partial_{Z_{j}}\right)^{k_{1}} \\
& \quad \cdot\left(\epsilon_{0}^{r\left(s_{1}+k_{0}\right)-s_{2}} \mathbb{A}_{s_{1}, s_{2}, k_{0}, k_{1}, k_{2}}\left(Z_{0}, X\right)\left(\partial_{X}^{k_{2}} U\right)\left(Z_{0}, \ldots, Z_{l}, X, \epsilon\right)\right) \\
& \quad+\sum_{\left(l_{0}, l_{1}\right) \in \mathscr{A}_{2}, l_{1} \geq 2} \sum_{m_{1} \in J_{\left(l_{0}, l_{1}\right)}} C_{10}\left(1+\sum_{j=0}^{l} Z_{j} \partial_{Z_{j}}\right)^{h r_{1}} \\
& \cdot \epsilon_{0}^{r\left(l_{0}+l_{1}-1\right)-m_{1}} C_{11}^{l_{1}} \mathbb{B}_{m_{1}, l_{0}, l_{1}}\left(Z_{0}, X\right)\left(U\left(Z_{0}, \ldots, Z_{l}, X, \epsilon\right)\right)^{l_{1}}
\end{aligned}
$$

for given initial data

$$
\begin{array}{r}
\left(\partial_{X}^{j} U\right)\left(Z_{0}, \ldots, Z_{l}, 0, \epsilon\right)=\sum_{\beta_{0} \geq 0, \ldots, \beta_{l} \geq 0} w_{\beta_{0}, \ldots, \beta_{l}, j}(\epsilon) \frac{Z_{0}^{\beta_{0}} \cdots Z_{l}^{\beta_{l}}}{\beta_{0} ! \cdots \beta_{l} !}, \\
0 \leq j \leq S-1
\end{array}
$$

for all $\epsilon \in D\left(0, \epsilon_{0}\right) \backslash\{0\}$.

Proposition 18. Under the assumption that

$$
S>k_{2}+b\left(s_{1}+k_{0}+2\right)
$$


for all $\left(k_{0}, k_{1}, k_{2}\right) \in \mathscr{A}_{1}$ and all $\left(s_{1}, s_{2}\right) \in I_{\left(k_{0}, k_{1}, k_{2}\right)}$, there exists a formal series

$$
U\left(Z_{0}, \ldots, Z_{l}, X, \epsilon\right)=\sum_{\underline{\beta}=\left(\beta_{0}, \ldots, \beta_{l+1}\right) \in \mathbb{N}^{l+2}} U_{\underline{\beta}}(\epsilon) \frac{Z_{0}^{\beta_{0}} \cdots Z_{l}^{\beta_{l}} X^{\beta_{l+1}}}{\beta_{0} ! \cdots \beta_{l} ! \beta_{l+1} !}
$$

solution of (114) and (115), where the coefficients $U_{\beta}(\epsilon)$ satisfy the following recursion:

$$
\begin{aligned}
& \frac{U_{\left(\beta_{0}, \ldots, \beta_{l}, \beta_{l+1}+S\right)}(\epsilon)}{\beta_{0} ! \cdots \beta_{l} ! \beta_{l+1} !} \\
& =\sum_{\left(k_{0}, k_{1}, k_{2}\right) \in \mathscr{A}_{1}} \sum_{\left(s_{1}, s_{2}\right) \in I_{\left(k_{0}, k_{1}, k_{2}\right)}} C_{10}\left(1+\sum_{j=0}^{l} \beta_{j}\right)^{h r_{1}} \\
& \sum_{\substack{\beta_{0}^{1}+\beta_{0}^{2}=\beta_{0} \\
\beta_{l+1}^{1}+\beta_{l+1}^{2}=\beta_{l+1}}} \frac{A_{s_{1}, s_{2}, k_{0}, k_{1}, k_{2}, \beta_{0}^{1}, \beta_{l+1}^{1}}}{\beta_{0}^{1} ! \beta_{l+1}^{1} !} \\
& \cdot \frac{U_{\left(\beta_{0}^{2}, \beta_{1}, \ldots, \beta_{l}, \beta_{l+1}^{2}+k_{2}\right)}(\epsilon)}{\beta_{0}^{2} ! \beta_{1} ! \cdots \beta_{l} ! \beta_{l+1}^{2} !} \epsilon_{0}^{r\left(s_{1}+k_{0}\right)-s_{2}} \\
& \cdot\left(\left(\sum_{j=0}^{l+1} \beta_{j}+S\right)^{b\left(s_{1}+k_{0}\right)}\left(\frac{\left(s_{1}+k_{0}\right) e^{-1}}{\sigma\left(S-k_{2}\right)}\right)^{s_{1}+k_{0}}\right. \\
& +\left(\sum_{j=0}^{l+1} \beta_{j}+S\right)^{b\left(s_{1}+k_{0}+2\right)} \\
& \left.\left(\frac{\left(s_{1}+k_{0}+2\right) e^{-1}}{\sigma\left(S-k_{2}\right)}\right)^{s_{1}+k_{0}+2}\right) \\
& \cdot\left(\beta_{0}+\sum_{j=1}^{l} \beta_{j}\left|\xi_{j}\right|\right)^{k_{1}} \\
& +\sum_{\left(l_{0}, l_{1}\right) \in \mathscr{A}_{2}, l_{1} \geq 2} \sum_{m_{1} \in J_{l_{0}, l_{1}}} C_{10}\left(1+\sum_{j=0}^{l} \beta_{j}\right)^{h r_{1}} \\
& \sum_{\beta_{0}^{-1}+\beta_{0}^{0}+\cdots+\beta_{0}^{l_{1}-1}=\beta_{0}} \frac{B_{m_{1}, l_{0}, l_{1}, \beta_{0}^{-1}, \beta_{l+1}^{-1}}}{\beta_{0}^{-1} ! \beta_{l+1}^{-1} !} \\
& \beta_{j}^{0}+\cdots+\beta_{j}^{l_{1}-1}=\beta_{j}, 1 \leq j \leq l \\
& \beta_{l+1}^{-1}+\beta_{l+1}^{0}+\cdots+\beta_{l+1}^{l_{1}-1}=\beta_{l+1} \\
& \cdot C_{11}^{l_{1}} \epsilon_{0}^{r\left(l_{0}+l_{1}-1\right)-m_{1}} \frac{\prod_{j=0}^{l_{1}-1} U_{\left(\beta_{0}^{j}, \ldots, \beta_{l+1}^{j}\right)}(\epsilon)}{\prod_{j=0}^{l_{1}-1} \beta_{0}^{j} ! \cdots \beta_{l+1}^{j} !}
\end{aligned}
$$

for all $\beta_{j} \geq 0,0 \leq j \leq l+1$, and all $\epsilon \in D\left(0, \epsilon_{0}\right) \backslash\{0\}$.
Proposition 19. Under assumption (116) with the additional condition that

$$
\begin{gathered}
r\left(s_{1}+k_{0}\right) \geq s_{2}, \\
r\left(l_{0}+l_{1}-1\right) \geq m_{1}, \quad l_{1} \geq 2,
\end{gathered}
$$

for all $\left(k_{0}, k_{1}, k_{2}\right) \in \mathscr{A}_{1}$, all $\left(s_{1}, s_{2}\right) \in I_{\left(k_{0}, k_{1}, k_{2}\right)}$, and all $\left(l_{0}, l_{1}\right) \in$ $\mathscr{A}_{2}, l_{1} \geq 2$ and $m_{1} \in J_{l_{0}, l_{1}}$, the following inequalities hold:

$$
w_{\underline{\beta}}(\epsilon) \leq U_{\underline{\beta}}(\epsilon)
$$

for all $\beta \in \mathbb{N}^{l+2}$, all $\epsilon \in D\left(0, \epsilon_{0}\right) \backslash\{0\}$.

Proof. By assumption (115), we know that

$$
U_{\left(\beta_{0}, \ldots, \beta_{l}, j\right)}(\epsilon)=w_{\left(\beta_{0}, \ldots, \beta_{l}, j\right)}(\epsilon)
$$

for all $0 \leq j \leq S-1$, all $\left(\beta_{0}, \ldots, \beta_{l}\right) \in \mathbb{N}^{l+1}$, and all $\epsilon \epsilon$ $D\left(0, \epsilon_{0}\right) \backslash\{0\}$. Therefore, we get our result by using induction from inequalities (108) and equalities (118).

In the next proposition we give sufficient conditions for the formal series solutions of the Cauchy problem (92), (93) in order to define actual holomorphic functions with exponential bound estimates.

Proposition 20. We make the assumption that (119) holds. We also assume that

$$
\begin{gathered}
S>b\left(s_{1}+k_{0}+2\right)+k_{2}, \\
S \geq h r_{1}+b\left(s_{1}+k_{0}+2\right)+k_{1}+k_{2}
\end{gathered}
$$

for all $\left(k_{0}, k_{1}, k_{2}\right) \in \mathscr{A}_{1}$ and all $\left(s_{1}, s_{2}\right) \in I_{\left(k_{0}, k_{1}, k_{2}\right)}$.

We choose two real numbers $\rho_{1}^{\prime}, M^{0}>0$ such that

$$
M^{0}>2(l+2) \exp \left(\rho_{1}^{\prime} \max _{j=0}^{l}\left|\xi_{j}\right|\right)
$$

and we take $\bar{X}^{0}>0$ and $\bar{Z}_{j}^{0}>M^{0}$, for $0 \leq j \leq l$. We assume that the formal series

$$
\begin{array}{r}
\varphi_{j}\left(Z_{0}, \ldots, Z_{l}, \epsilon\right)=\sum_{\beta_{0}, \ldots, \beta_{l} \geq 0} w_{\left(\beta_{0}, \ldots, \beta_{l}, j\right)}(\epsilon) \frac{Z_{0}^{\beta_{0}} \cdots Z_{l}^{\beta_{l}}}{\beta_{0} ! \cdots \beta_{l} !}, \\
0 \leq j \leq S-1,
\end{array}
$$

belong to $G\left(\bar{Z}_{0}^{0}, \ldots, \bar{Z}_{l}^{0}, \bar{X}^{0}\right)$ for all $\epsilon \in D\left(0, \epsilon_{0}\right) \backslash\{0\}$ and that there exists a constant $C_{\varphi_{j}}$ such that $\sup _{\epsilon \in D\left(0, \epsilon_{0}\right) \backslash\{0\}}\left\|\varphi_{j}\right\|_{\left(\bar{Z}_{0}^{0}, \ldots, \bar{Z}_{l}^{0}, \bar{X}^{0}\right)} \leq C_{\varphi_{j}}$. As a consequence, the formal series (91) define holomorphic functions $V_{j}(\tau, z, \epsilon)$ on the product $S_{d} \times H_{\rho_{1}^{\prime}} \times D\left(0, \epsilon_{0}\right) \backslash\{0\}$ and satisfy the following estimates: there exists a constant $C_{12}>0$ (depending on $C_{\varphi_{j}}, l$ ) such that

$$
\left|V_{j}(\tau, z, \epsilon)\right| \leq C_{12}\left(1+\frac{|\tau|^{2}}{|\epsilon|^{2 r}}\right)^{-1} \exp \left(\frac{\sigma}{|\epsilon|^{r}} \zeta(b)|\tau|\right)
$$

for all $(\tau, z, \epsilon) \in S_{d} \times H_{\rho_{1}^{\prime}} \times D\left(0, \epsilon_{0}\right) \backslash\{0\}$, and all $0 \leq j \leq S-1$. 
Then, there exists $\delta>0$ (depending on $M^{0}, \bar{Z}_{j}^{0},\left|\xi_{j}\right|$ for $0 \leq j \leq l, \bar{X}^{0}, r_{1}, r_{2}, C_{\xi_{1}, \ldots, \xi_{l}}, S_{d}, h, \sigma, b, S, \mathbb{A}_{s_{1}, s_{2}, k_{0}, k_{1}, k_{2}}\left(Z_{0}, X\right)$ for $\left(k_{0}, k_{1}, k_{2}\right) \in \mathscr{A}_{1}$ and $\left(s_{1}, s_{2}\right) \in I_{\left(k_{0}, k_{1}, k_{2}\right)}$ and $\mathbb{B}_{m_{1}, l_{0}, l_{1}}\left(Z_{0}, X\right)$ for $\left.\left(l_{0}, l_{1}\right) \in \mathscr{A}_{2}, m_{1} \in J_{\left(l_{0}, l_{1}\right)}\right)$ such that if one assumes moreover that

$$
\left\|\varphi_{j}\left(Z_{0}, \ldots, Z_{l}, \epsilon\right)\right\|_{\left(\bar{Z}_{0}^{0}, \ldots, \bar{Z}_{l}^{0}, \bar{X}^{0}\right)}<\delta, \quad 0 \leq j \leq S-1,
$$

for all $\epsilon \in D\left(0, \epsilon_{0}\right) \backslash\{0\}$, the formal series (106), solution of (92) and (93), defines a holomorphic function $V(\tau, z, x, \epsilon)$ on the product $S_{d} \times H_{\rho_{1}^{\prime}} \times D\left(0, \rho_{1}\right) \times D\left(0, \epsilon_{0}\right) \backslash\{0\}$ for some $\rho_{1}>0$ and carries the next bound estimates: there exists a constant $C_{13}>0$ (depending on the same as for $\delta$ given above) with

$$
|V(\tau, z, x, \epsilon)| \leq C_{13}\left(1+\frac{|\tau|^{2}}{|\epsilon|^{2 r}}\right)^{-1} \exp \left(\frac{\sigma}{|\epsilon|^{r}} \zeta(b)|\tau|\right)
$$

for all $(\tau, z, x, \epsilon) \in S_{d} \times H_{\rho_{1}^{\prime}} \times D\left(0, \rho_{1}\right) \times D\left(0, \epsilon_{0}\right) \backslash\{0\}$.

Proof. Since $\varphi_{j}\left(Z_{0}, \ldots, Z_{l}, \epsilon\right)$ belongs to $G\left(\bar{Z}_{0}^{0}, \ldots, \bar{Z}_{l}^{0}, \bar{X}^{0}\right)$, we get a constant $C_{14}>0$ (depending on $C_{\varphi_{j}}$ ) such that

$$
\begin{aligned}
& \left\|V_{\left(\beta_{0}, \ldots, \beta_{l}, j\right)}(\tau, \epsilon)\right\|_{\left(\beta_{0}, \ldots, \beta_{l}, j\right), \epsilon, \sigma, r, \Omega_{\left(\beta_{0}, \ldots, \beta_{l}, j\right)}} \\
& \quad \leq C_{14}\left(\frac{1}{\bar{Z}_{0}^{0}}\right)^{\beta_{0}} \cdots\left(\frac{1}{\bar{Z}_{l}^{0}}\right)^{\beta_{l}}\left(\sum_{q=0}^{l} \beta_{q}\right) !
\end{aligned}
$$

for all $\beta_{0}, \ldots, \beta_{l} \geq 0$ and all $0 \leq j \leq S-1$. From the multinomial formula, we know that

$$
\left(\sum_{q=0}^{l} \beta_{q}\right) ! \leq(l+1)^{\sum_{q=0}^{l} \beta_{q}} \beta_{0} ! \cdots \beta_{l} !
$$

for all $\beta_{0}, \ldots, \beta_{l} \geq 0$. Therefore, from (128), we deduce that

$$
\begin{aligned}
\left|V_{\left(\beta_{0}, \ldots, \beta_{l}, j\right)}(\tau, \epsilon)\right| \leq & C_{14}\left(1+\frac{|\tau|^{2}}{|\epsilon|^{2 r}}\right)^{-1} \\
& \cdot \exp \left(\frac{\sigma}{|\epsilon|^{r}} r_{b}\left(\sum_{q=0}^{l} \beta_{q}+j\right)|\tau|\right) \\
& \cdot\left(\frac{l+1}{M^{0}}\right)^{\sum_{q=0}^{l} \beta_{q}} \beta_{0} ! \cdots \beta_{l} !
\end{aligned}
$$

for all $\beta_{0}, \ldots, \beta_{l} \geq 0,0 \leq j \leq S-1$, all $\tau \in \Omega_{\left(\beta_{0}, \ldots, \beta_{l}, j\right)}$, and all $\epsilon \in D\left(0, \epsilon_{0}\right) \backslash\{0\}$. From assumption (123), we deduce that the formal series (91) defines a holomorphic function $V_{j}(\tau, z, \epsilon)$ on the product $S_{d} \times H_{\rho_{1}^{\prime}} \times D\left(0, \epsilon_{0}\right) \backslash\{0\}$ and satisfies

$$
\begin{aligned}
\left|V_{j}(\tau, z, \epsilon)\right| \leq & C_{14}\left(1+\frac{|\tau|^{2}}{|\epsilon|^{2 r}}\right)^{-1} \exp \left(\frac{\sigma}{|\epsilon|^{r}} \zeta(b)|\tau|\right) \\
& \cdot \sum_{\beta_{0}, \ldots, \beta_{l} \geq 0}\left(\frac{(l+1) \exp \left(\rho_{1}^{\prime} \max _{q=0}^{l}\left|\xi_{q}\right|\right)}{M^{0}}\right)^{\sum_{q=0}^{l} \beta_{q}} \\
\leq & 2^{l+1} C_{14}\left(1+\frac{|\tau|^{2}}{|\epsilon|^{2 r}}\right)^{-1} \exp \left(\frac{\sigma}{|\epsilon|^{r}} \zeta(b)|\tau|\right)
\end{aligned}
$$

for all $(\tau, z, \epsilon) \in S_{d} \times H_{\rho_{1}^{\prime}} \times D\left(0, \epsilon_{0}\right) \backslash\{0\}$ and all $0 \leq j \leq S-1$.
Under assumptions (119) and (122) together with (126), we see that the hypotheses of Proposition 5 are fulfilled for the Cauchy problem (114), (115). Therefore, we deduce that the formal solution $U\left(Z_{0}, \ldots, Z_{l}, X, \epsilon\right)$ of (114), (115) constructed in Proposition 18 belongs to the space $G\left(\bar{Z}_{0}^{1}, \ldots, \bar{Z}_{l}^{1}, \bar{X}^{1}\right)$ for some $0<\bar{X}^{1}<\bar{X}^{0}$ and for some $\bar{Z}_{j}^{1}>M^{0}$. Moreover, we get a constant $C_{15}>0$ (depending on $\bar{Z}_{j}^{0}, \bar{Z}_{j}^{1},\left|\xi_{j}\right|$ for $0 \leq j \leq l, \bar{X}^{0}, r_{1}, r_{2}, C_{\xi_{1}, \ldots, \xi_{l}}, S_{d}, h, \sigma, b, S, \mathbb{A}_{s_{1}, s_{2}, k_{0}, k_{1}, k_{2}}\left(Z_{0}, X\right)$ for $\left(k_{0}, k_{1}, k_{2}\right) \in \mathscr{A}_{1}$ and $\left(s_{1}, s_{2}\right) \in I_{\left(k_{0}, k_{1}, k_{2}\right)}$ and $B_{m_{1}, l_{0}, l_{1}}\left(Z_{0}, X\right)$ for $\left.\left(l_{0}, l_{1}\right) \in \mathscr{A}_{2}, m_{1} \in J_{\left(l_{0}, l_{1}\right)}\right)$ such that

$$
\left\|U\left(Z_{0}, \ldots, Z_{l}, X, \epsilon\right)\right\|_{\left(\bar{Z}_{0}^{1}, \ldots, \bar{Z}_{l}^{1}\right)} \leq \delta C_{15}
$$

for all $\epsilon \in D\left(0, \epsilon_{0}\right) \backslash\{0\}$. In particular, we deduce that

$$
\begin{aligned}
& \left|U_{\left(\beta_{0}, \ldots, \beta_{l+1}\right)}(\epsilon)\right| \\
& \quad \leq \delta C_{15}\left(\frac{1}{\bar{Z}_{0}^{1}}\right)^{\beta_{0}} \cdots\left(\frac{1}{\bar{Z}_{l}^{1}}\right)^{\beta_{l}}\left(\frac{1}{\bar{X}^{1}}\right)^{\beta_{l+1}}\left(\sum_{j=0}^{l+1} \beta_{j}\right) !
\end{aligned}
$$

for all $\beta_{0}, \ldots, \beta_{l+1} \geq 0$. Gathering (120) and (133) yields

$$
\begin{aligned}
& \left\|V_{\underline{\beta}}(\tau, \epsilon)\right\|_{\underline{\beta}, \epsilon, \sigma, r, \Omega_{\underline{\beta}}} \\
& \quad \leq \delta C_{15}\left(\frac{1}{\bar{Z}_{0}^{1}}\right)^{\beta_{0}} \cdots\left(\frac{1}{\bar{Z}_{l}^{1}}\right)^{\beta_{l}}\left(\frac{1}{\bar{X}^{1}}\right)^{\beta_{l+1}}\left(\sum_{j=0}^{l+1} \beta_{j}\right) !
\end{aligned}
$$

for all $\beta=\left(\beta_{0}, \ldots, \beta_{l+1}\right) \in \mathbb{N}^{l+2}$. Again by the multinomial formula, we have that

$$
\left(\sum_{j=0}^{l+1} \beta_{j}\right) ! \leq(l+2)^{\sum_{j=0}^{l+1} \beta_{j}} \beta_{0} ! \cdots \beta_{l+1} !
$$

for all $\beta_{0}, \ldots, \beta_{l+1} \geq 0$. Hence, from (134), we get that

$$
\begin{aligned}
\left|V_{\underline{\beta}}(\tau, \epsilon)\right| \leq & \delta C_{15}\left(1+\frac{|\tau|^{2}}{|\epsilon|^{2 r}}\right)^{-1} \exp \left(\frac{\sigma}{|\epsilon|^{r}} r_{b}(\underline{\beta})|\tau|\right) \\
& \cdot\left(\frac{l+2}{M^{0}}\right)^{\sum_{j=0}^{l} \beta_{j}}\left(\frac{l+2}{\bar{X}^{1}}\right)^{\beta_{l+1}} \beta_{0} ! \cdots \beta_{l+1} !
\end{aligned}
$$

for all $\beta_{0}, \ldots, \beta_{l+1} \geq 0$, all $\tau \in \Omega_{\beta}$, and all $\epsilon \in D\left(0, \epsilon_{0}\right) \backslash\{0\}$. We deduce that the formal series (106) defines a holomorphic 
function $V(\tau, z, x, \epsilon)$ on the product $S_{d} \times H_{\rho_{1}^{\prime}} \times D\left(0, \bar{X}^{1} /(2(l+\right.$ 2)) $) \times D\left(0, \epsilon_{0}\right) \backslash\{0\}$ and satisfies

$$
\begin{aligned}
& |V(\tau, z, x, \epsilon)| \\
& \leq \delta C_{15}\left(1+\frac{|\tau|^{2}}{|\epsilon|^{2 r}}\right)^{-1} \exp \left(\frac{\sigma}{|\epsilon|^{r}} \zeta(b)|\tau|\right) \\
& \left.\quad \sum_{\beta_{0}, \ldots, \beta_{l+1} \geq 0}\left(\frac{(l+2) \exp \left(\rho_{1}^{\prime} \max _{j=0}^{l}\left|\xi_{j}\right|\right)}{M^{0}}\right)\right)^{\sum_{j=0}^{l} \beta_{j}} \\
& \cdot\left(\frac{(l+2)|x|}{\bar{X}^{1}}\right)^{\beta_{l+1}} \\
& \leq 2^{l+2} \delta C_{15}\left(1+\frac{|\tau|^{2}}{|\epsilon|^{2 r}}\right)^{-1} \exp \left(\frac{\sigma}{|\epsilon|^{r}} \zeta(b)|\tau|\right)
\end{aligned}
$$

for all $(\tau, z, x, \epsilon) \in S_{d} \times H_{\rho_{1}^{\prime}} \times D\left(0, \bar{X}^{1} /(2(l+2))\right) \times D\left(0, \epsilon_{0}\right) \backslash$ $\{0\}$.

\section{Analytic Solutions in a Complex Parameter of a Singular Cauchy Problem}

3.1. Laplace Transform and Asymptotic Expansions. We recall the definition of Borel summability of formal series with coefficients in a Banach space; see [18].

Definition 21. A formal series

$$
\widehat{X}(t)=\sum_{j=0}^{\infty} \frac{a_{j}}{j !} t^{j} \in \mathbb{E}[[t]]
$$

with coefficients in a Banach space $\left(\mathbb{E},\|\cdot\|_{\mathbb{E}}\right)$ is said to be 1summable with respect to $t$ in the direction $d \in[0,2 \pi)$ if

(i) there exists $\rho \in \mathbb{R}_{+}$such that the following formal series, called formal Borel transform of $\widehat{X}$ of order 1

$$
\mathscr{B}(\widehat{X})(\tau)=\sum_{j=0}^{\infty} \frac{a_{j} \tau^{j}}{(j !)^{2}} \in \mathbb{E}[[\tau]],
$$

is absolutely convergent for $|\tau|<\rho$;

(ii) there exists $\delta>0$ such that the series $\mathscr{B}(\widehat{X})(\tau)$ can be analytically continued with respect to $\tau$ in a sector $S_{d, \delta}=\left\{\tau \in \mathbb{C}^{*}:|d-\arg (\tau)|<\delta\right\}$. Moreover, there exist $C>0$ and $K>0$ such that

$$
\|\mathscr{B}(\widehat{X})(\tau)\|_{\mathbb{E}} \leq C e^{K|\tau|}
$$

for all $\tau \in S_{d, \delta}$.

If this is so, the vector valued Laplace transform of order 1 of $\mathscr{B}(\widehat{X})(\tau)$ in the direction $d$ is defined by

$$
\mathscr{L}^{d}(\mathscr{B}(\widehat{X}))(t)=t^{-1} \int_{L_{\gamma}} \mathscr{B}(\widehat{X})(\tau) e^{-(\tau / t)} d \tau,
$$

along a half-line $L_{\gamma}=\mathbb{R}_{+} e^{i \gamma} \subset S_{d, \delta} \cup\{0\}$, where $\gamma$ depends on $t$ and is chosen in such a way that $\cos (\gamma-\arg (t)) \geq \delta_{1}>0$, for some fixed $\delta_{1}$, for all $t$ in any sector

$$
S_{d, \theta, R}=\left\{t \in \mathbb{C}^{*}:|t|<R,|d-\arg (t)|<\frac{\theta}{2}\right\},
$$

where $\pi<\theta<\pi+2 \delta$ and $0<R<\delta_{1} / K$. The function $\mathscr{L}^{d}(\mathscr{B}(\widehat{X}))(t)$ is called the 1-sum of the formal series $\widehat{X}(t)$ in the direction $d$ and defines a holomorphic bounded function on the sector $S_{d, \theta, R}$. Moreover, it has the formal series $\widehat{X}(t)$ as Gevrey asymptotic expansion of order 1 with respect to $t$ on $S_{d, \theta, R}$. This means that, for all $\theta_{1}<\theta$, there exist $C, M>0$ such that

$$
\left\|\mathscr{L}^{d}(\mathscr{B}(\widehat{X}))(t)-\sum_{p=0}^{n-1} \frac{a_{p}}{p !} t^{p}\right\|_{\mathbb{E}} \leq C M^{n} n !|t|^{n}
$$

for all $n \geq 1$ and all $t \in S_{d, \theta_{1}, R}$.

In the next proposition, we give some well-known identities for the Borel transform that will be useful in the sequel.

Proposition 22. Let $\widehat{X}(t)=\sum_{n \geq 0} a_{n} t^{n} / n$ ! and $\widehat{G}(t)=$ $\sum_{n \geq 0} b_{n} t^{n} / n$ ! be formal series in $\mathbb{E}[[t]]$. We have the following equalities as formal series in $\mathbb{E}[[\tau]]$ :

$$
\begin{gathered}
\left(\tau \partial_{\tau}^{2}+\partial_{\tau}\right)(\mathscr{B}(\widehat{X})(\tau))=\mathscr{B}\left(\partial_{t} \widehat{X}(t)\right)(\tau), \\
\partial_{\tau}^{-1}(\mathscr{B}(\widehat{X}))(\tau)=\mathscr{B}(t \widehat{X}(t))(\tau), \\
\tau \mathscr{B}(\widehat{X})(\tau)=\mathscr{B}\left(\left(t^{2} \partial_{t}+t\right) \widehat{X}(t)\right)(\tau), \\
\int_{0}^{\tau}(\mathscr{B} \widehat{X})(\tau-s)(\mathscr{B} \widehat{G})(s) d s \\
=\mathscr{B}(t \widehat{X}(t) \widehat{G}(t))(\tau)
\end{gathered}
$$

Proof. By a direct computation, we have the following expansions from which Proposition 22 follows:

$$
\begin{gathered}
\partial_{t} \widehat{X}(t)=\sum_{n \geq 0} a_{n+1} \frac{t^{n}}{n !}, \\
\left(\tau \partial_{\tau}^{2}+\partial_{\tau}\right)(\mathscr{B}(\widehat{X})(\tau))=\sum_{n \geq 0} a_{n+1} \frac{\tau^{n}}{(n !)^{2}}, \\
t \widehat{X}(t)=\sum_{n \geq 1} n a_{n-1} \frac{t^{n}}{n !}, \\
\partial_{\tau}^{-1}(\mathscr{B}(\widehat{X}))(\tau)=\sum_{n \geq 1} n a_{n-1} \frac{\tau^{n}}{(n !)^{2}}, \\
\left(t^{2} \partial_{t}+t\right) \widehat{X}(t)=\sum_{n \geq 1} n^{2} a_{n-1} \frac{t^{n}}{n !},
\end{gathered}
$$




$$
\begin{gathered}
\tau \mathscr{B}(\widehat{X})(\tau)=\sum_{n \geq 1} n^{2} a_{n-1} \frac{\tau^{n}}{(n !)^{2}}, \\
t \widehat{X}(t) \widehat{G}(t)=\sum_{n \geq 1}\left(\sum_{l+m=n-1} \frac{n !}{l ! m !} a_{l} b_{m}\right) \frac{t^{n}}{n !}, \\
\int_{0}^{\tau}(\mathscr{B} \widehat{X})(\tau-s)(\mathscr{B} \widehat{G})(s) d s \\
=\sum_{n \geq 1}\left(\sum_{l+m=n-1} \frac{n !}{l ! m !} a_{l} b_{m}\right) \frac{\tau^{n}}{(n !)^{2}} .
\end{gathered}
$$

3.2. Analytic Solutions of Some Singular Cauchy Problem. Let $S, r_{1}, r_{2} \geq 1$ be integers. Let $\mathcal{S}$ be a finite subset of $\mathbb{N}^{4}$ and $\mathcal{N}$ a finite subset of $\mathbb{N}^{2}$. For all $\left(s, k_{0}, k_{1}, k_{2}\right) \in \mathcal{S}$ and all integers $\beta_{0}, \beta_{l+1} \geq 0$, we denote by $b_{s, k_{0}, k_{1}, k_{2}, \beta_{0}, \beta_{l+1}}(\epsilon)$ some holomorphic function on $D\left(0, \epsilon_{0}\right)$ which satisfies the next estimates: there exist constants $\rho, \rho^{\prime}>0, \mathfrak{b}_{s, k_{0}, k_{1}, k_{2}}>0$ with

$$
\begin{aligned}
& \sup _{\epsilon \in D\left(0, \epsilon_{0}\right)}\left|b_{s, k_{0}, k_{1}, k_{2}, \beta_{0}, \beta_{l+1}}(\epsilon)\right| \\
& \quad \leq \mathfrak{b}_{s, k_{0}, k_{1}, k_{2}}\left(\frac{e^{-\rho^{\prime}}}{2}\right)^{\beta_{0}}\left(\frac{1}{2 \rho}\right)^{\beta_{l+1}} \beta_{0} ! \beta_{l+1} !
\end{aligned}
$$

for all $\beta_{0}, \beta_{l+1} \geq 0$. Likewise, for all $\left(l_{0}, l_{1}\right) \in \mathcal{N}$ and all integers $\beta_{0}, \beta_{l+1} \geq 0$, we denote by $c_{l_{0}, l_{1}, \beta_{0}, \beta_{l+1}}(\epsilon)$ some holomorphic function on $D\left(0, \epsilon_{0}\right)$ with the following estimates: there exists a constant $\mathfrak{c}_{l_{0}, l_{1}}>0$ with

$$
\sup _{\epsilon \in D\left(0, \epsilon_{0}\right)}\left|c_{l_{0}, l_{1}, \beta_{0}, \beta_{l+1}}(\epsilon)\right| \leq \mathfrak{c}_{l_{0}, l_{1}}\left(\frac{e^{-\rho^{\prime}}}{2}\right)^{\beta_{0}}\left(\frac{1}{2 \rho}\right)^{\beta_{l+1}} \beta_{0} ! \beta_{l+1} !
$$

for all $\beta_{0}, \beta_{l+1} \geq 0$.

For all $\left(s, k_{0}, k_{1}, k_{2}\right) \in \mathcal{S}$ and all $\left(l_{0}, l_{1}\right) \in \mathcal{N}$, we consider the series

$$
\begin{aligned}
b_{s, k_{0}, k_{1}, k_{2}}(z, x, \epsilon) & =\sum_{\beta_{0}, \beta_{l+1} \geq 0} b_{s, k_{0}, k_{1}, k_{2}, \beta_{0}, \beta_{l+1}}(\epsilon) \frac{e^{i z \beta_{0}}}{\beta_{0} !} \frac{x^{\beta_{l+1}}}{\beta_{l+1} !}, \\
c_{l_{0}, l_{1}}(z, x, \epsilon) & =\sum_{\beta_{0}, \beta_{l+1} \geq 0} c_{l_{0}, l_{1}, \beta_{0}, \beta_{l+1}}(\epsilon) \frac{e^{i z \beta_{0}}}{\beta_{0} !} \frac{x^{\beta_{l+1}}}{\beta_{l+1} !}
\end{aligned}
$$

which define bounded holomorphic functions on $H_{\rho^{\prime}} \times$ $D(0, \rho) \times D\left(0, \epsilon_{0}\right)$.

We consider the following singular Cauchy problem:

$$
\begin{gathered}
\left(\left(T^{2} \partial_{T}+T\right)^{r_{2}}+\left(-i \partial_{z}+1\right)^{r_{1}}\right) \partial_{x}^{S} Y_{U_{d}, D\left(0, \epsilon_{0}\right) \backslash\{0\}}(T, z, x, \epsilon) \\
=\sum_{\left(s, k_{0}, k_{1}, k_{2}\right) \in \mathcal{S}} b_{s, k_{0}, k_{1}, k_{2}}(z, x, \epsilon) \epsilon^{r\left(k_{0}-s\right)} T^{\mathcal{S}} \\
\cdot\left(\partial_{T}^{k_{0}} \partial_{z}^{k_{1}} \partial_{x}^{k_{2}} Y_{U_{d}, D\left(0, \epsilon_{0}\right) \backslash\{0\}}\right)(T, z, x, \epsilon)
\end{gathered}
$$

$$
\begin{array}{r}
+\sum_{\left(l_{0}, l_{1}\right) \in \mathcal{N}} \mathcal{c}_{l_{0}, l_{1}}(z, x, \epsilon) \epsilon^{-r\left(l_{0}+l_{1}-1\right)} T^{l_{0}+l_{1}-1} \\
\cdot\left(Y_{U_{d}, D\left(0, \epsilon_{0}\right) \backslash\{0\}}(T, z, x, \epsilon)\right)^{l_{1}}
\end{array}
$$

for given initial data

$$
\begin{array}{r}
\left(\partial_{x}^{j} Y_{U_{d}, D\left(0, \epsilon_{0}\right) \backslash\{0\}}\right)(T, z, 0, \epsilon)=Y_{U_{d}, D\left(0, \epsilon_{0}\right) \backslash\{0\}, j}(T, z, \epsilon), \\
0 \leq j \leq S-1 .
\end{array}
$$

The initial conditions are constructed as follows. Let $U_{d}$ be an unbounded sector such that

$$
\arg (\tau) \neq \frac{2 k+1}{r_{2}} \pi
$$

for all $0 \leq k \leq r_{2}-1$ and all $\tau \in U_{d}$. For all $0 \leq j \leq S-1$ and all $\left(\beta_{0}, \ldots, \beta_{l}\right) \in \mathbb{N}^{l+1}$, let $V_{U_{d},\left(\beta_{0}, \ldots, \beta_{l}, j\right)}(\tau, \epsilon)$ be a function such that

$$
V_{U_{d},\left(\beta_{0}, \ldots, \beta_{l}, j\right)}(\tau, \epsilon) \in E_{\left(\beta_{0}, \ldots, \beta_{l}, j\right), \epsilon, \sigma, r, D\left(0, \rho_{\left(\beta_{0}, \ldots, \beta_{l}, j\right)}\right) \cup U_{d}}
$$

for all $\epsilon \in D\left(0, \epsilon_{0}\right) \backslash\{0\}$.

We choose two real numbers $\rho_{1}^{\prime}, M^{0}>0$ such that

$$
M^{0}>2(l+2) \exp \left(\rho_{1}^{\prime} \max _{j=0}^{l}\left|\xi_{j}\right|\right)
$$

and we take $\bar{X}^{0}>0$ and $\bar{Z}_{j}^{0}>M^{0}$, for all $0 \leq j \leq l$. We make the assumption that the formal series

$$
\begin{gathered}
\varphi_{d, j}\left(Z_{0}, \ldots, Z_{l}, \epsilon\right) \\
=\sum_{\beta_{0}, \ldots, \beta_{l} \geq 0}\left\|V_{U_{d},\left(\beta_{0}, \ldots, \beta_{l}, j\right)}(\tau, \epsilon)\right\|_{\left(\beta_{0}, \ldots, \beta_{l}, j\right), \epsilon, \sigma, r, D\left(0, \rho_{\left(\beta_{0}, \ldots, \beta_{l}, j\right)}\right) \cup U_{d}} \\
\cdot \frac{Z_{0}^{\beta_{0}} \cdots Z_{l}^{\beta_{l}}}{\beta_{0} ! \cdots \beta_{l} !}
\end{gathered}
$$

belongs to the Banach space $G\left(\bar{Z}_{0}^{0}, \ldots, \bar{Z}_{l}^{0}, \bar{X}^{0}\right)$ for all $\epsilon \epsilon$ $D\left(0, \epsilon_{0}\right) \backslash\{0\}$ and all $0 \leq j \leq S-1$. Moreover, we assume that there exists a constant $C_{\varphi_{d, j}}>0$ such that $\sup _{\epsilon \in D\left(0, \epsilon_{0}\right) \backslash\{0\}}\left\|\varphi_{d, j}\right\|_{\left(\bar{Z}_{0}^{0}, \ldots, \bar{Z}_{l}^{0}, \bar{X}^{0}\right)} \leq C_{\varphi_{d, j}}$.

Let

$$
V_{U_{d},\left(\beta_{0}, \ldots, \beta_{l}, j\right)}(\tau, \epsilon)=\sum_{m \geq 0} \frac{\chi_{m,\left(\beta_{0}, \ldots, \beta_{l}, j\right)}(\epsilon)}{(m !)^{2}} \tau^{m}
$$

be its Taylor expansion with respect to $\tau$ on $D\left(0, \rho_{\left(\beta_{0}, \ldots, \beta_{l}, j\right)}\right)$, for all $\epsilon \in D\left(0, \epsilon_{0}\right) \backslash\{0\}$. We consider the formal series

$$
\widehat{Y}_{\left(\beta_{0}, \ldots, \beta_{l}, j\right)}(T, \epsilon)=\sum_{m \geq 0} \frac{\chi_{m,\left(\beta_{0}, \ldots, \beta_{l}, j\right)}(\epsilon)}{m !} T^{m}
$$

for all $\epsilon \in D\left(0, \epsilon_{0}\right) \backslash\{0\}$. For all $\left(\beta_{0}, \ldots, \beta_{l}, j\right)$ we define $Y_{U_{d}, D\left(0, \epsilon_{0}\right) \backslash\{0\},\left(\beta_{0}, \ldots, \beta_{l}, j\right)}(T, \epsilon)$ as the 1-sum of $\widehat{Y}_{\left(\beta_{0}, \ldots, \beta_{l}, j\right)}(T, \epsilon)$ in 
the direction $d$. From the fact that $\tau \mapsto V_{U_{d},\left(\beta_{0}, \ldots, \beta_{l}, j\right)}(\tau, \epsilon)$ belongs to $E_{\left(\beta_{0}, \ldots, \beta_{l}, j\right), \epsilon, \sigma, r, D\left(0, \rho_{\left(\beta_{0}, \ldots, \beta_{l}, j\right)}\right) \cup U_{d}}$, for all $\epsilon \in D\left(0, \epsilon_{0}\right) \backslash$ $\{0\}$, we get that $Y_{U_{d}, D\left(0, \epsilon_{0}\right) \backslash\{0\},\left(\beta_{0}, \ldots, \beta_{l}, j\right)}(T, \epsilon)$ defines a holomorphic function for all $T \in U_{d, \theta, h|\epsilon|^{r}}$ and all $\epsilon \in D\left(0, \epsilon_{0}\right) \backslash\{0\}$, where

$$
U_{d, \theta, h|\epsilon|^{r}}=\left\{T \in \mathbb{C}:|T|<h|\epsilon|^{r},|d-\arg (T)|<\frac{\theta}{2}\right\},
$$

for some $\theta>\pi$ and some constant $h>0$ (independent of $\epsilon$ and $\left.\beta_{0}, \ldots, \beta_{l}\right)$, for all $0 \leq j \leq S-1$. The initial data are defined as the formal series

$$
\begin{aligned}
& Y_{U_{d}, D\left(0, \epsilon_{0}\right) \backslash\{0\}, j}(T, z, \epsilon) \\
& =\sum_{\beta_{0}, \ldots, \beta_{l} \geq 0} Y_{U_{d}, D\left(0, \epsilon_{0}\right) \backslash\{0\},\left(\beta_{0}, \ldots, \beta_{l}, j\right)}(T, \epsilon) \frac{\exp \left(i z\left(\sum_{j=0}^{l} \beta_{j} \xi_{j}\right)\right)}{\beta_{0} ! \cdots \beta_{l} !}, \\
& 0 \leq j \leq S-1,
\end{aligned}
$$

which actually define holomorphic functions on the domain $U_{d, \theta, h|\epsilon|^{r}} \times H_{\rho_{1}^{\prime}} \times D\left(0, \epsilon_{0}\right) \backslash\{0\}$. Indeed from hypotheses (153) and (154) and the multinomial formula (129), following the first part of the proof of Proposition 20, we get that there exists a constant $C_{16}>0$ such that

$$
\begin{aligned}
& \left|\sum_{\beta_{0}, \ldots, \beta_{l} \geq 0} V_{U_{d},\left(\beta_{0}, \ldots, \beta_{l}, j\right)}(\tau, \epsilon) \frac{\exp \left(i z\left(\sum_{j=0}^{l} \beta_{j} \xi_{j}\right)\right)}{\beta_{0} ! \cdots \beta_{l} !}\right| \\
& \leq C_{16}\left(1+\frac{|\tau|^{2}}{|\epsilon|^{2 r}}\right)^{-1} \exp \left(\frac{\sigma}{|\epsilon|^{r}} \zeta(b)|\tau|\right) \\
& \quad \sum_{\beta_{0}, \ldots, \beta_{l} \geq 0}\left(\frac{(l+1) \exp \left(\rho_{1}^{\prime} \max _{j=0}^{l}\left|\xi_{j}\right|\right)}{M^{0}}\right)^{\sum_{j=0}^{l} \beta_{j}} \\
& \leq 2^{l+1} C_{16}\left(1+\frac{|\tau|^{2}}{|\epsilon|^{2 r}}\right)^{-1} \exp \left(\frac{\sigma}{|\epsilon|^{r}} \zeta(b)|\tau|\right)
\end{aligned}
$$

for all $\tau \in U_{d}, z \in H_{\rho_{1}^{\prime}}$ and $\epsilon \in D\left(0, \epsilon_{0}\right) \backslash\{0\}$.

We get the following result.

Proposition 23. Let the initial data be constructed as above. We make the following assumptions. For all $\left(s, k_{0}, k_{1}, k_{2}\right) \in \mathcal{S}$ and all $\left(l_{0}, l_{1}\right) \in \mathcal{N}$, we have that

$$
\begin{gathered}
s \geq 2 k_{0}, \\
s>k_{2}, \\
S>b\left(s-k_{0}+2\right)+k_{2}, \\
S \geq h r_{1}+b\left(s-k_{0}+2\right)+k_{1}+k_{2}, \\
l_{1} \geq 2 .
\end{gathered}
$$

Then, there exists a constant $I>0$ (independent of $\epsilon$ ) such that if one assumes that

$$
\left\|\varphi_{d, j}\left(Z_{0}, \ldots, Z_{l}, \epsilon\right)\right\|_{\left(\bar{Z}_{0}^{0}, \ldots, \bar{Z}_{l}^{0}, \bar{X}^{0}\right)}<I
$$

where $\varphi_{d, j}$ is defined in (154), for all $\epsilon \in D\left(0, \epsilon_{0}\right) \backslash\{0\}$, the problem (149), (150) has a solution

$$
\begin{aligned}
Y_{U_{d}, D\left(0, \epsilon_{0}\right) \backslash\{0\}}(T, z, x, \epsilon) & \\
= & \sum_{\beta_{0}, \ldots, \beta_{l}, \beta_{l+1} \geq 0} Y_{U_{d}, D\left(0, \epsilon_{0}\right) \backslash\{0\},\left(\beta_{0}, \ldots, \beta_{l}, \beta_{l+1}\right)}(T, \epsilon) \\
& \frac{\exp \left(i z\left(\sum_{j=0}^{l} \beta_{j} \xi_{j}\right)\right)}{\beta_{0} ! \cdots \beta_{l} !} \frac{x^{\beta_{l+1}}}{\beta_{l+1} !}
\end{aligned}
$$

which defines a bounded holomorphic function on $U_{d, \theta, h^{\prime}|\epsilon|^{r}} \times$ $H_{\rho_{1}^{\prime}} \times D\left(0, \rho_{1}\right)$ for some $h^{\prime}>0$ and $\rho_{1}>0$, for all $\epsilon \in D\left(0, \epsilon_{0}\right) \backslash$ $\{0\}$. Moreover, each function $Y_{U_{d}, D\left(0, \epsilon_{0}\right) \backslash\{0\},\left(\beta_{0}, \ldots, \beta_{l}, \beta_{l+1}\right)}(T, \epsilon)$ can be written as a Laplace transform of order 1 in the direction $d$ of a function $\tau \mapsto V_{U_{d},\left(\beta_{0}, \ldots, \beta_{l}, \beta_{l+1}\right)}(\tau, \epsilon)$ which is holomorphic on $D\left(0, \rho_{\left(\beta_{0}, \ldots, \beta_{l}, \beta_{l+1}\right)}\right) \cup U_{d}$ and satisfies the estimates: there exist two constants $C_{17}>0, K_{17}>0$ (both independent of $\epsilon$ ) such that

$$
\begin{aligned}
& \left|V_{U_{d},\left(\beta_{0}, \ldots, \beta_{l}, \beta_{l+1}\right)}(\tau, \epsilon)\right| \\
& \leq C_{17} \exp \left(\frac{\sigma}{|\epsilon|^{r}} \zeta(b)|\tau|\right)\left(\frac{l+2}{M^{0}}\right)^{\sum_{j=0}^{l} \beta_{j}} K_{17}^{\beta_{l+1}} \beta_{0} ! \cdots \beta_{l+1} !
\end{aligned}
$$

for all $\tau \in D\left(0, \rho_{\left(\beta_{0}, \ldots, \beta_{l}, \beta_{l+1}\right)}\right) \cup U_{d}$ and $\epsilon \in D\left(0, \epsilon_{0}\right) \backslash\{0\}$.

Proof. One considers a formal series

$$
\begin{aligned}
& \widehat{Y}(T, z, x, \epsilon) \\
& =\sum_{\beta_{0}, \ldots, \beta_{l}, \beta_{l+1} \geq 0} \widehat{Y}_{\left(\beta_{0}, \ldots, \beta_{l}, \beta_{l+1}\right)}(T, \epsilon) \frac{\exp \left(i z\left(\sum_{j=0}^{l} \beta_{j} \xi_{j}\right)\right)}{\beta_{0} ! \cdots \beta_{l} !} \frac{x^{\beta_{l+1}}}{\beta_{l+1} !}
\end{aligned}
$$

solution of (149), with initial data

$$
\begin{aligned}
& \left(\partial_{x}^{j} \widehat{Y}\right)(T, z, 0, \epsilon) \\
& =\widehat{Y}_{j}(T, z, \epsilon) \\
& =\sum_{\beta_{0}, \ldots, \beta_{l} \geq 0} \widehat{Y}_{\left(\beta_{0}, \ldots, \beta_{l}, j\right)}(T, \epsilon) \frac{\exp \left(i z\left(\sum_{j=0}^{l} \beta_{j} \xi_{j}\right)\right)}{\beta_{0} ! \cdots \beta_{l} !}, \\
& 0 \leq j \leq S-1,
\end{aligned}
$$

for all $\epsilon \in D\left(0, \epsilon_{0}\right) \backslash\{0\}$ where $\widehat{Y}_{\left(\beta_{0}, \ldots, \beta_{l}, j\right)}(T, \epsilon)$ are defined in (156). We consider the formal Borel transform of $\widehat{Y}(T, z, x, \epsilon)$ of order 1 with respect to $T$ denoted by

$$
\begin{aligned}
& \widehat{V}(\tau, z, x, \epsilon) \\
& =\sum_{\beta_{0}, \ldots, \beta_{l}, \beta_{l+1} \geq 0} \widehat{V}_{\left(\beta_{0}, \ldots, \beta_{l}, \beta_{l+1}\right)}(\tau, \epsilon) \frac{\exp \left(i z\left(\sum_{j=0}^{l} \beta_{j} \xi_{j}\right)\right)}{\beta_{0} ! \cdots \beta_{l} !} \frac{x^{\beta_{l+1}}}{\beta_{l+1} !},
\end{aligned}
$$


where, by construction, $\widehat{V}_{\left(\beta_{0}, \ldots, \beta_{l}, \beta_{l+1}\right)}(\tau, \epsilon)$ is the Borel transform of order 1 with respect to $T$ of the formal series $\widehat{Y}_{\left(\beta_{0}, \ldots, \beta_{l}, \beta_{l+1}\right)}(T, \epsilon)$, for all $\epsilon \in D\left(0, \epsilon_{0}\right) \backslash\{0\}$.

From the identities of Proposition 22, we get that $\widehat{V}(\tau, z, x, \epsilon)$ satisfies the following singular Cauchy problem:

$$
\begin{aligned}
& \left(\tau^{r_{2}}+\left(-i \partial_{z}+1\right)^{r_{1}}\right) \partial_{x}^{S} \widehat{V}(\tau, z, x, \epsilon) \\
& =\sum_{\left(s, k_{0}, k_{1}, k_{2}\right) \in \mathcal{S}} b_{s, k_{0}, k_{1}, k_{2}}(z, x, \epsilon) \epsilon^{r\left(k_{0}-s\right)} \partial_{\tau}^{-s} \\
& \quad \cdot\left(\tau \partial_{\tau}^{2}+\partial_{\tau}\right)^{k_{0}} \partial_{z}^{k_{1}} \partial_{x}^{k_{2}} \widehat{V}(\tau, z, x, \epsilon) \\
& \quad+\sum_{\left(l_{0}, l_{1}\right) \in \mathcal{N}} c_{l_{0}, l_{1}}(z, x, \epsilon) \epsilon^{-r\left(l_{0}+l_{1}-1\right)} \partial_{\tau}^{-l_{0}}(\widehat{V}(\tau, z, x, \epsilon))^{* l_{1}}
\end{aligned}
$$

with initial data

$$
\begin{aligned}
&\left(\partial_{x}^{j} \widehat{V}\right)(\tau, z, 0, \epsilon) \\
&=\sum_{\beta_{0}, \ldots, \beta_{l} \geq 0} V_{\left(\beta_{0}, \ldots, \beta_{l}, j\right)}(\tau, \epsilon) \frac{\exp \left(i z\left(\sum_{j=0}^{l} \beta_{j} \xi_{j}\right)\right)}{\beta_{0} ! \cdots \beta_{l} !}, \\
& 0 \leq j \leq S-1,
\end{aligned}
$$

where $V_{\left(\beta_{0}, \ldots, \beta_{l}, j\right)}(\tau, \epsilon)$ are defined in (152), for all $\epsilon \in D\left(0, \epsilon_{0}\right) \backslash$ $\{0\}$. In the following, we rewrite (167) using the two following technical lemmas. Their proofs can be found in [17, Lemmas 5 and 6 ]. Therefore we omit them.

Lemma 24. For all $k_{0} \geq 1$, there exist constants $a_{k, k_{0}} \in \mathbb{N}$, $k_{0} \leq k \leq 2 k_{0}$, such that

$$
\left(\tau \partial_{\tau}^{2}+\partial_{\tau}\right)^{k_{0}} u(\tau)=\sum_{k=k_{0}}^{2 k_{0}} a_{k, k_{0}} \tau^{k-k_{0}} \partial_{\tau}^{k} u(\tau)
$$

for all holomorphic functions $u: \Omega \rightarrow \mathbb{C}$ on an open set $\Omega \subset$ $\mathbb{C}$.

Lemma 25. Let $a, b, c \geq 0$ be positive integers such that $a \geq b$ and $a \geq c$. We put $\delta=a+b-c$. Then, for all holomorphic functions $u: \Omega \rightarrow \mathbb{C}$, the function $\partial_{\tau}^{-a}\left(\tau^{b} \partial_{\tau}^{c} u(\tau)\right)$ can be written in the form

$$
\partial_{\tau}^{-a}\left(\tau^{b} \partial_{\tau}^{c} u(\tau)\right)=\sum_{\left(b^{\prime}, c^{\prime}\right) \in \mathcal{O}_{\delta}} \alpha_{b^{\prime}, c^{\prime}} \tau^{b^{\prime}} \partial_{\tau}^{c^{\prime}} u(\tau)
$$

where $\mathcal{O}_{\delta}$ is a finite subset of $\mathbb{Z}^{2}$ such that, for all $\left(b^{\prime}, c^{\prime}\right) \in \mathcal{O}_{\delta}$, $b^{\prime}-c^{\prime}=\delta, b^{\prime} \geq 0, c^{\prime} \leq 0$, and $\alpha_{b^{\prime}, c^{\prime}} \in \mathbb{Z}$.
Using the latter Lemmas 24 and 25 together with assumption (160), we can rewrite (167) in the form

$$
\begin{aligned}
& \left(\tau^{r_{2}}+\left(-i \partial_{z}+1\right)^{r_{1}}\right) \partial_{x}^{S} \widehat{V}(\tau, z, x, \epsilon) \\
& =\sum_{\left(s, k_{0}, k_{1}, k_{2}\right) \in \mathcal{S}} b_{s, k_{0}, k_{1}, k_{2}}(z, x, \epsilon) \epsilon^{r\left(k_{0}-s\right)} \\
& \\
& \quad\left(\sum_{\left(r^{\prime}, p^{\prime}\right) \in \mathcal{O}_{s-k_{0}}} \alpha_{\left.r^{\prime}, p^{\prime} \tau^{r^{\prime}} \partial_{\tau}^{-p^{\prime}} \partial_{z}^{k_{1}} \partial_{x}^{k_{2}} \widehat{V}(\tau, z, x, \epsilon)\right)}\right. \\
& \quad+\sum_{\left(l_{0}, l_{1}\right) \in \mathcal{N}} \mathcal{C}_{l_{0}, l_{1}}(z, x, \epsilon) \epsilon^{-r\left(l_{0}+l_{1}-1\right)} \partial_{\tau}^{-l_{0}}(\widehat{V}(\tau, z, x, \epsilon))^{* l_{1}},
\end{aligned}
$$

where $\mathcal{O}_{s-k_{0}}$ is a finite subset of $\mathbb{N}^{2}$ such that, for all $\left(r^{\prime}, p^{\prime}\right) \in$ $\mathcal{O}_{s-k_{0}}$, we have $r^{\prime}+p^{\prime}=s-k_{0}$ and $\alpha_{r^{\prime}, p^{\prime}} \in \mathbb{Z}$, for the given initial data

$$
\begin{aligned}
&\left(\partial_{x}^{j} \widehat{V}\right)(\tau, z, 0, \epsilon) \\
&=\sum_{\beta_{0}, \ldots, \beta_{l} \geq 0} V_{\left(\beta_{0}, \ldots, \beta_{l}, j\right)}(\tau, \epsilon) \frac{\exp \left(i z\left(\sum_{j=0}^{l} \beta_{j} \xi_{j}\right)\right)}{\beta_{0} ! \cdots \beta_{l} !}, \\
& 0 \leq j \leq S-1 .
\end{aligned}
$$

From assumption (160), we deduce that assumptions (119) and (122) of Proposition 20 are fulfilled for (171). Hence, from Proposition 20, we deduce the existence of a constant $I>0$ (independent of $\epsilon$ ) such that if inequality (161) holds, then the formal series $\widehat{V}(\tau, z, x, \epsilon)$ solution of (171) and (172) defines a holomorphic function $V_{U_{d}}(\tau, z, x, \epsilon)$ on the product $U_{d} \times$ $H_{\rho_{1}^{\prime}} \times D\left(0, \rho_{1}\right) \times D\left(0, \epsilon_{0}\right) \backslash\{0\}$ which satisfies the next bound estimates: there exists a constant $C_{18}>0$ such that

$$
\left|V_{U_{d}}(\tau, z, x, \epsilon)\right| \leq C_{18}\left(1+\frac{|\tau|^{2}}{|\epsilon|^{2 r}}\right)^{-1} \exp \left(\frac{\sigma}{|\epsilon|^{r}} \zeta(b)|\tau|\right)
$$

for all $(\tau, z, x, \epsilon) \in U_{d} \times H_{\rho_{1}^{\prime}} \times D\left(0, \rho_{1}\right) \times D\left(0, \epsilon_{0}\right) \backslash\{0\}$. Moreover, from the proof of Proposition 20 (especially formula (136)), we also get that each formal series $\widehat{V}_{\left(\beta_{0}, \ldots, \beta_{l}, \beta_{l+1}\right)}(\tau, \epsilon)$ defines a holomorphic function $V_{U_{d},\left(\beta_{0}, \ldots, \beta_{l}, \beta_{l+1}\right)}(\tau, \epsilon)$ on $\left(U_{d} \cup\right.$ $\left.D\left(0, \rho_{\left(\beta_{0}, \ldots, \beta_{l+1}\right)}\right)\right) \times D\left(0, \epsilon_{0}\right) \backslash\{0\}$ with the following estimates: there exist two constants $C_{19}>0, K_{19}>0$ such that

$$
\begin{aligned}
\left|V_{U_{d},\left(\beta_{0}, \ldots, \beta_{l}, \beta_{l+1}\right)}(\tau, \epsilon)\right| \\
\leq I C_{19} \exp \left(\frac{\sigma}{|\epsilon|^{r}} r_{b}\left(\left(\beta_{0}, \ldots, \beta_{l+1}\right)\right)|\tau|\right)\left(\frac{l+2}{M^{0}}\right)^{\sum_{j=0}^{l} \beta_{j}} \\
\quad \cdot K_{19}^{\beta_{l+1}} \beta_{0} ! \cdots \beta_{l+1} !
\end{aligned}
$$

for all $\tau \in D\left(0, \rho_{\left(\beta_{0}, \ldots, \beta_{l+1}\right)}\right) \cup U_{d}$ and $\epsilon \in D\left(0, \epsilon_{0}\right) \backslash\{0\}$. From (174), we get that each formal series $\widehat{Y}_{\left(\beta_{0}, \ldots, \beta_{l}, \beta_{l+1}\right)}(T, \epsilon)$ is 1-summable with respect to the fact that $T$ is the direction 
$d$ and its 1 -sum denoted by $Y_{U_{d}, D\left(0, \epsilon_{0}\right) \backslash\{0\},\left(\beta_{0}, \ldots, \beta_{l}, \beta_{l+1}\right)}(T, \epsilon)$ can be written as Laplace transform of order 1 in the direction $d$ of the function $\tau \mapsto V_{U_{d},\left(\beta_{0}, \ldots, \beta_{l}, \beta_{l+1}\right)}(\tau, \epsilon)$. Moreover, again by (174), we deduce that the series

$$
\begin{aligned}
& Y_{U_{d}, D\left(0, \epsilon_{0}\right) \backslash\{0\}}(T, z, x, \epsilon) \\
&=\sum_{\beta_{0}, \ldots, \beta_{l}, \beta_{l+1} \geq 0} Y_{U_{d}, D\left(0, \epsilon_{0}\right) \backslash\{0\},\left(\beta_{0}, \ldots, \beta_{l}, \beta_{l+1}\right)}(T, \epsilon) \\
& \cdot \frac{\exp \left(i z\left(\sum_{j=0}^{l} \beta_{j} \xi_{j}\right)\right)}{\beta_{0} ! \cdots \beta_{l} !} \frac{x^{\beta_{l+1}}}{\beta_{l+1} !}
\end{aligned}
$$

defines a bounded holomorphic function on $U_{d, \theta, h^{\prime}|\epsilon|^{r}} \times H_{\rho_{1}^{\prime}} \times$ $D\left(0, \rho_{1}\right)$ for some $h^{\prime}>0$ and $\rho_{1}>0$, for all $\epsilon \in D\left(0, \epsilon_{0}\right) \backslash\{0\}$. Finally, from the algebraic properties of the $\kappa$-summation procedure (see [18] Section 6.3) since $\widehat{Y}(T, z, x, \epsilon)$ formally solves (149), we deduce that $Y_{U_{d}, D\left(0, \epsilon_{0}\right) \backslash\{0\}}(T, z, x, \epsilon)$ is an actual solution of the Cauchy problem (149), (150).

\section{Formal Series Solutions and Gevrey Asymptotic Expansion in a Complex Parameter for the Main Cauchy Problem}

4.1. Analytic Solutions in a Complex Parameter for the Main Cauchy Problem. We recall the definition of a good covering.

Definition 26. Let $v \geq 2$ be an integer. For all $0 \leq i \leq v-1$, we consider an open sector $\mathscr{E}_{i}$ with vertex at 0 and with radius $\epsilon_{0}$. We assume that these sectors are three by three disjoint and that $\mathscr{E}_{i+1} \cap \mathscr{E}_{i} \neq \emptyset$, for all $0 \leq i \leq v-1$, where by convention we define $\mathscr{E}_{v}=\mathscr{E}_{0}$. Moreover, we assume that $\cup_{0 \leq i \leq v-1} \mathscr{E}_{i}=$ $\mathcal{U} \backslash\{0\}$, where $\mathcal{U}$ is some neighborhood of 0 in $\mathbb{C}$. Such a set of sectors $\left\{\mathscr{E}_{i}\right\}_{0 \leq i \leq \nu-1}$ is called a good covering in $\mathbb{C}^{*}$.

Definition 27. Let $\left\{\mathscr{E}_{i}\right\}_{0 \leq i \leq \nu-1}$ be a good covering in $\mathbb{C}^{*}$. Let $r>0$ be a positive real number and let $r_{2} \geq 1$ be some integer. Let $\mathscr{T}$ be an open sector with vertex at 0 with radius $r_{\mathscr{T}}>0$. We consider the following family of open sectors:

$$
U_{d_{i}, \theta, \epsilon_{0}^{r} r_{\mathscr{T}}}=\left\{t \in \mathbb{C}^{*}:|t|<\epsilon_{0}^{r} r_{\mathscr{T}},\left|d_{i}-\arg (t)\right|<\frac{\theta}{2}\right\},
$$

where $d_{i} \in \mathbb{R}, 0 \leq i \leq \nu-1$ and $\theta>\pi$, which satisfy the following properties.

(1) For all $0 \leq i \leq v-1, d_{i} \neq \pi\left((2 k+1) / r_{2}\right)$ for all $0 \leq$ $k \leq r_{2}-1$.

(2) For all $0 \leq i \leq \nu-1$, all $t \in \mathscr{T}$, and all $\epsilon \in \mathscr{E}_{i}$, we have that $\epsilon^{r} t \in U_{d_{i}, \theta, \epsilon_{0}^{r} r_{\mathscr{T}}}$.

Under the above settings, we say that the family $\left\{\left\{U_{d_{i}, \theta, \epsilon_{0}^{r} r_{\mathscr{T}}}\right\}_{0 \leq i \leq \nu-1}, \mathscr{T}\right\}$ is associated with the good covering $\left\{\mathscr{E}_{i}\right\}_{0 \leq i \leq \nu-1}$.

Let $S \geq 1$ be an integer. Let $\mathcal{S}$ be a finite subset of $\mathbb{N}^{4}$, and let $\mathscr{N}$ be a finite subset of $\mathbb{N}^{2}$.

As in the previous section, for all $\left(s, k_{0}, k_{1}, k_{2}\right) \in \mathcal{S}$ and all integers $\beta_{0}, \beta_{l+1} \geq 0$, we denote by $b_{s, k_{0}, k_{1}, k_{2}, \beta_{0}, \beta_{l+1}}(\epsilon)$ some holomorphic function on $D\left(0, \epsilon_{0}\right)$ which satisfies the next estimates: there exist constants $\rho, \rho^{\prime}>0, \mathfrak{b}_{s, k_{0}, k_{1}, k_{2}}>0$ with

$$
\begin{aligned}
& \sup _{\epsilon \in D\left(0, \epsilon_{0}\right)}\left|b_{s, k_{0}, k_{1}, k_{2}, \beta_{0}, \beta_{l+1}}(\epsilon)\right| \\
& \quad \leq \mathfrak{b}_{s, k_{0}, k_{1}, k_{2}}\left(\frac{e^{-\rho^{\prime}}}{2}\right)^{\beta_{0}}\left(\frac{1}{2 \rho}\right)^{\beta_{l+1}} \beta_{0} ! \beta_{l+1} !
\end{aligned}
$$

for all $\beta_{0}, \beta_{l+1} \geq 0$. Likewise, for all $\left(l_{0}, l_{1}\right) \in \mathcal{N}$ and all integers $\beta_{0}, \beta_{l+1} \geq 0$, we denote by $c_{l_{0}, l_{1}, \beta_{0}, \beta_{l+1}}(\epsilon)$ some holomorphic function on $D\left(0, \epsilon_{0}\right)$ with the following estimates: there exists a constant $\mathfrak{c}_{l_{0}, l_{1}}>0$ with

$$
\sup _{\epsilon \in D\left(0, \epsilon_{0}\right)}\left|c_{l_{0}, l_{1}, \beta_{0}, \beta_{l+1}}(\epsilon)\right| \leq \mathfrak{c}_{l_{0}, l_{1}}\left(\frac{e^{-\rho^{\prime}}}{2}\right)^{\beta_{0}}\left(\frac{1}{2 \rho}\right)^{\beta_{l+1}} \beta_{0} ! \beta_{l+1} !
$$

for all $\beta_{0}, \beta_{l+1} \geq 0$.

For all $\left(s, k_{0}, k_{1}, k_{2}\right) \in \mathcal{S}$ and all $\left(l_{0}, l_{1}\right) \in \mathcal{N}$, we consider the series

$$
\begin{aligned}
b_{s, k_{0}, k_{1}, k_{2}}(z, x, \epsilon) & =\sum_{\beta_{0}, \beta_{l+1} \geq 0} b_{s, k_{0}, k_{1}, k_{2}, \beta_{0}, \beta_{l+1}}(\epsilon) \frac{e^{i z \beta_{0}}}{\beta_{0} !} \frac{x^{\beta_{l+1}}}{\beta_{l+1} !} \\
\mathcal{c}_{l_{0}, l_{1}}(z, x, \epsilon) & =\sum_{\beta_{0}, \beta_{l+1} \geq 0} c_{l_{0}, l_{1}, \beta_{0}, \beta_{l+1}}(\epsilon) \frac{e^{i z \beta_{0}}}{\beta_{0} !} \frac{x^{\beta_{l+1}}}{\beta_{l+1} !}
\end{aligned}
$$

which define bounded holomorphic functions on $H_{\rho^{\prime}} \times$ $D(0, \rho) \times D\left(0, \epsilon_{0}\right)$. Let $\left\{\mathscr{E}_{i}\right\}_{0 \leq i \leq \nu-1}$ be a good covering in $\mathbb{C}^{*}$ and let $r_{1}, r_{2}, r_{3} \geq 1$ be three integers. We put $r=r_{3} / r_{2}$.

For all $0 \leq i \leq v-1$, we consider the following Cauchy problem:

$$
\begin{aligned}
& \left(\epsilon^{r_{3}}\left(t^{2} \partial_{t}+t\right)^{r_{2}}+\left(-i \partial_{z}+1\right)^{r_{1}}\right) \partial_{x}^{S} X_{i}(t, z, x, \epsilon) \\
& =\sum_{\left(s, k_{0}, k_{1}, k_{2}\right) \in \mathcal{S}} b_{s, k_{0}, k_{1}, k_{2}}(z, x, \epsilon) t^{s}\left(\partial_{t}^{k_{0}} \partial_{z}^{k_{1}} \partial_{x}^{k_{2}} X_{i}\right)(t, z, x, \epsilon) \\
& \quad+\sum_{\left(l_{0}, l_{1}\right) \in \mathcal{N}} c_{l_{0}, l_{1}}(z, x, \epsilon) t^{l_{0}+l_{1}-1}\left(X_{i}(t, z, x, \epsilon)\right)^{l_{1}}
\end{aligned}
$$

for given initial data

$$
\left(\partial_{x}^{j} X_{i}\right)(t, z, 0, \epsilon)=\Xi_{i, j}(t, z, \epsilon), \quad 0 \leq j \leq S-1,
$$

where the functions $\Xi_{i, j}$ are constructed as follows. We consider a family of sectors $\left\{\left\{U_{d_{i}, \theta, \epsilon_{0}^{r} r_{\mathscr{T}}}\right\}_{0 \leq i \leq \nu-1}, \mathscr{T}\right\}$ associated with the good covering $\left\{\mathscr{E}_{i}\right\}_{0 \leq i \leq v-1}$. For all $0 \leq i \leq \nu-1$, let $U_{d_{i}}$ be an unbounded open sector centered at 0 , with bisecting direction $d_{i}$ and with aperture $n_{i}>\theta-\pi$. We choose $\theta$ and $n_{i}$ in such a way that

$$
\arg (\tau) \neq \pi \frac{2 k+1}{r_{2}}
$$


for all $\tau \in U_{d_{i}}$, all $0 \leq i \leq v-1$, and all $0 \leq k \leq r_{2}-1$. For all $0 \leq i \leq \nu-1$ and all $0 \leq j \leq S-1$, we define

$$
\Xi_{i, j}(t, z, \epsilon)=Y_{U_{d_{i}}, D\left(0, \epsilon_{0}\right) \backslash\{0\}, j}\left(\epsilon^{r} t, z, \epsilon\right),
$$

where $Y_{U_{d_{i}}, D\left(0, \epsilon_{0}\right) \backslash\{0\}, j}(T, z, \epsilon)$ is given by expression (158) and constructed as in the beginning of Section 3.2 with the help of a family of functions $V_{U_{d_{i}},\left(\beta_{0}, \ldots, \beta_{l}, j\right)}(\tau, \epsilon)$, for $\left(\beta_{0}, \ldots, \beta_{l}\right) \epsilon$ $\mathbb{N}^{l+1}, 0 \leq j \leq S-1$, satisfying (152).

We make the additional assumption that, for all $\left(\beta_{0}, \ldots, \beta_{l}\right) \in \mathbb{N}^{l+1}, 0 \leq j \leq S-1$, there exists a holomorphic function $\tau \mapsto V_{\left(\beta_{0}, \ldots, \beta_{l}, j\right)}(\tau, \epsilon)$ on $D\left(0, \rho_{\left(\beta_{0}, \ldots, \beta_{l+1}\right)}\right)$ for all $\epsilon \in D\left(0, \epsilon_{0}\right) \backslash\{0\}$ such that

$$
V_{U_{d_{i}},\left(\beta_{0}, \ldots, \beta_{l}, j\right)}(\tau, \epsilon)=V_{\left(\beta_{0}, \ldots, \beta_{l}, j\right)}(\tau, \epsilon)
$$

for all $0 \leq i \leq \nu-1$, all $0 \leq j \leq S-1$, all $\tau \in D\left(0, \rho_{\left(\beta_{0}, \ldots, \beta_{l+1}\right)}\right)$, and all $\epsilon \in D\left(0, \epsilon_{0}\right) \backslash\{0\}$.

Moreover, we assume that the series $\varphi_{d_{i}, j}\left(Z_{0}, \ldots, Z_{l}, \epsilon\right)$ defined in (154) belong to the Banach space $G\left(\bar{Z}_{0}^{0}, \ldots, \bar{Z}_{l}^{0}, \bar{X}^{0}\right)$ for all $\epsilon \in D\left(0, \epsilon_{0}\right) \backslash\{0\}$, where $\bar{X}^{0}>0$ and $\bar{Z}_{j}^{0}, 0 \leq j \leq l$, are chosen in such a way that $\bar{Z}_{j}^{0}>M^{0}$ for $M^{0}>0$ that fulfills inequality (153) for some real number $\rho_{1}^{\prime}>0$.

By construction, $\Xi_{i, j}(t, z, \epsilon)$ defines a holomorphic function on $\mathscr{T} \times H_{\rho_{1}^{\prime}} \times \mathscr{E}_{i}$, for all $0 \leq i \leq v-1$ and all $0 \leq j \leq S-1$, for well-chosen radius $r_{\mathscr{T}}>0$ and aperture $\theta$.

Proposition 28. Let the initial data (181) be constructed as above. We make the following assumptions: for all $\left(s, k_{0}, k_{1}, k_{2}\right) \in \mathcal{S}$ and all $\left(l_{0}, l_{1}\right) \in \mathcal{N}$, we have that

$$
\begin{gathered}
s \geq 2 k_{0}, \\
S>k_{2}, \\
S>b\left(s-k_{0}+2\right)+k_{2}, \\
S \geq h r_{1}+b\left(s-k_{0}+2\right)+k_{1}+k_{2}, \\
l_{1} \geq 2 .
\end{gathered}
$$

Then, there exists a constant $I>0$ (independent of $\epsilon$ ) such that if one assumes that

$$
\left\|\varphi_{d_{i}, j}\left(Z_{0}, \ldots, Z_{l}, \epsilon\right)\right\|_{\left(\bar{Z}_{0}^{0}, \ldots, \bar{Z}_{l}^{0}, \bar{X}^{0}\right)}<I
$$

for all $0 \leq i \leq \nu-1$, for all $\epsilon \in D\left(0, \epsilon_{0}\right) \backslash\{0\}$, the problem (180), (181) has a solution $X_{i}(t, z, x, \epsilon)$ which is holomorphic and bounded on $\left(\mathscr{T} \cap D\left(0, h^{\prime}\right)\right) \times H_{\rho_{1}^{\prime}} \times D\left(0, \rho_{1}\right) \times \mathscr{E}_{i}$, for some $\rho_{1}>0$. Moreover, there exist constants $K_{23}, M_{23}>0$ and $0<$ $h^{\prime \prime}<h^{\prime}$ such that

$$
\begin{aligned}
& \sup _{t \in \mathscr{T} \cap D\left(0, h^{\prime \prime}\right), z \in H_{\rho_{1}^{\prime}}, x \in D\left(0, \rho_{1}\right)}\left|X_{i+1}(t, z, x, \epsilon)-X_{i}(t, z, x, \epsilon)\right| \\
& \leq K_{23} \exp \left(-\frac{M_{23}}{|\epsilon|^{r_{3} /\left(h r_{1}+r_{2}\right)}}\right)
\end{aligned}
$$

for all $\epsilon \in \mathscr{E}_{i+1} \cap \mathscr{E}_{i}$ and all $0 \leq i \leq \nu-1$ (where by convention $\left.X_{v}=X_{0}\right)$, provided that $\epsilon_{0}>0$ is small enough.

Proof. For all $0 \leq i \leq v-1$, we consider the singular Cauchy problem (149) with initial data

$$
\begin{array}{r}
\left(\partial_{x}^{j} Y_{U_{d_{i}}, D\left(0, \epsilon_{0}\right) \backslash\{0\}}\right)(T, z, 0, \epsilon)=Y_{U_{d_{i}}, D\left(0, \epsilon_{0}\right) \backslash\{0\}, j}(T, z, \epsilon), \\
0 \leq j \leq S-1 .
\end{array}
$$

Bearing in mind hypothesis (185) and assumption (186), we see that the assumptions of Proposition 23 are all fulfilled for the problem (149), (188), which, therefore, possesses a solution $(T, z, x) \mapsto Y_{U_{d_{i}}, D\left(0, \epsilon_{0}\right) \backslash\{0\}}(T, z, x, \epsilon)$, holomorphic and bounded on $U_{d_{i}, \theta, h^{\prime}|\epsilon|^{r}} \times H_{\rho_{1}^{\prime}} \times D\left(0, \rho_{1}\right)$ for some $h^{\prime}>0$ and $\rho_{1}>0$, for all $\epsilon \in D\left(0, \epsilon_{0}\right) \backslash\{0\}$. Now, we put

$$
X_{i}(t, z, x, \epsilon)=Y_{U_{d_{i}}, D\left(0, \epsilon_{0}\right) \backslash\{0\}}\left(\epsilon^{r} t, z, x, \epsilon\right)
$$

which defines a holomorphic and bounded function on $\left(\mathscr{T} \cap D\left(0, h^{\prime}\right)\right) \times H_{\rho_{1}^{\prime}} \times D\left(0, \rho_{1}\right) \times \mathscr{E}_{i}$, for all $0 \leq i \leq$ $\nu-1$, by construction of $\mathscr{T}$ and $\mathscr{E}_{i}$ in Definition 27. Since $Y_{U_{d_{i}}, D\left(0, \epsilon_{0}\right) \backslash\{0\}}(T, z, x, \epsilon)$ solves the problem (149), (188), one can check that $X_{i}(t, z, x, \epsilon)$ solves the problem (180), (181) on $\left(\mathscr{T} \cap D\left(0, h^{\prime}\right)\right) \times H_{\rho_{1}^{\prime}} \times D\left(0, \rho_{1}\right) \times \mathscr{E}_{i}$, for all $0 \leq i \leq \nu-1$.

In the next step of the proof, we show estimates (187). Let $0 \leq i \leq v-1$. Using Proposition 23, we can write the function $X_{i}(t, z, x, \epsilon)$ as follows:

$$
\begin{aligned}
& X_{i}(t, z, x, \epsilon) \\
& =\sum_{\beta_{0}, \ldots, \beta_{l}, \beta_{l+1} \geq 0} X_{i,\left(\beta_{0}, \ldots, \beta_{l+1}\right)}(t, \epsilon) \frac{\exp \left(i z\left(\sum_{j=0}^{l} \beta_{j} \xi_{j}\right)\right)}{\beta_{0} ! \cdots \beta_{l} !} \frac{x^{\beta_{l+1}}}{\beta_{l+1} !}
\end{aligned}
$$

where

$$
X_{i,\left(\beta_{0}, \ldots, \beta_{l+1}\right)}(t, \epsilon)=\frac{1}{\epsilon^{r} t} \int_{L_{\gamma_{i}}} V_{U_{d_{i},\left(\beta_{0}, \ldots, \beta_{l+1}\right)}}(\tau, \epsilon) e^{-\tau /\left(\epsilon^{r} t\right)} d \tau
$$

with integration path $L_{\gamma_{i}}=\mathbb{R}_{+} e^{\sqrt{-1} \gamma_{i}} \subset U_{d_{i}}$ and such that $\tau \mapsto V_{U_{\left.d_{i}, \beta_{0}, \ldots, \beta_{l+1}\right)}}(\tau, \epsilon)$ are holomorphic functions on $D\left(0, \rho_{\left(\beta_{0}, \ldots, \beta_{l+1}\right)}\right) \cup U_{d_{i}}$, for all $\epsilon \in D\left(0, \epsilon_{0}\right) \backslash\{0\}$, and satisfy the estimates: there exist constants $C_{20}, K_{20}>0$, which satisfy $\rho_{1} K_{20}<1$, with

$$
\begin{aligned}
& \left|V_{U_{d_{i}},\left(\beta_{0}, \ldots, \beta_{l}, \beta_{l+1}\right)}(\tau, \epsilon)\right| \\
& \leq C_{20} \exp \left(\frac{\sigma}{|\epsilon|^{r}} \zeta(b)|\tau|\right)\left(\frac{l+2}{M^{0}}\right)^{\sum_{j=0}^{l} \beta_{j}} K_{20}^{\beta_{l+1}} \beta_{0} ! \cdots \beta_{l+1} !
\end{aligned}
$$

for all $\tau \in D\left(0, \rho_{\left(\beta_{0}, \ldots, \beta_{l}, \beta_{l+1}\right)}\right) \cup U_{d_{i}}$ and $\epsilon \in D\left(0, \epsilon_{0}\right) \backslash\{0\}$. Moreover, from assumption (184), we deduce with the help 
of recursion (107), which is satisfied for the coefficients of the formal solution $\widehat{V}(\tau, z, x, \epsilon)$ of the problem (171), (172), that, for all $\left(\beta_{0}, \ldots, \beta_{l}, \beta_{l+1}\right) \in \mathbb{N}^{l+2}$, there exists a holomorphic function $\tau \mapsto V_{\left(\beta_{0}, \ldots, \beta_{l}, \beta_{l+1}\right)}(\tau, \epsilon)$ on $D\left(0, \rho_{\left(\beta_{0}, \ldots, \beta_{l}, \beta_{l+1}\right)}\right)$ for all $\epsilon \in D\left(0, \epsilon_{0}\right) \backslash\{0\}$ such that

$$
V_{U_{d_{i}},\left(\beta_{0}, \ldots, \beta_{l}, \beta_{l+1}\right)}(\tau, \epsilon)=V_{\left(\beta_{0}, \ldots, \beta_{l}, \beta_{l+1}\right)}(\tau, \epsilon)
$$

for all $\tau \in D\left(0, \rho_{\left(\beta_{0}, \ldots, \beta_{l}, \beta_{l+1}\right)}\right)$, all $\epsilon \in D\left(0, \epsilon_{0}\right) \backslash\{0\}$, and all $0 \leq i \leq v-1$.

We show the following.

Lemma 29. There exist constants $0<h^{\prime \prime}<h^{\prime}, C_{21}, K_{21}$, $M_{21}>0$ (independent of $\epsilon$ ), which satisfy $\rho_{1} K_{21}<1$, such that

$$
\begin{aligned}
& \sup _{t \in \mathscr{T} \cap D\left(0, h^{\prime \prime}\right)}\left|X_{i+1,\left(\beta_{0}, \ldots, \beta_{l+1}\right)}(t, \epsilon)-X_{i,\left(\beta_{0}, \ldots, \beta_{l+1}\right)}(t, \epsilon)\right| \\
& \leq C_{21}\left(\frac{l+2}{M^{0}}\right)^{\sum_{j=0}^{l} \beta_{j}} K_{21}^{\beta_{l+1}} \beta_{0} ! \cdots \beta_{l+1} ! e^{-M_{21}\left(\rho_{\left(\beta_{0}, \ldots, \beta_{l+1}\right)} /|\epsilon|^{r}\right)}
\end{aligned}
$$

for all $\epsilon \in \mathscr{E}_{i+1} \cap \mathscr{E}_{i}$, all $0 \leq i \leq \nu-1$, and all $\left(\beta_{0}, \ldots, \beta_{l+1}\right) \epsilon$ $\mathbb{N}^{l+2}\left(\right.$ where by convention $\left.X_{v,\left(\beta_{0}, \ldots, \beta_{l+1}\right)}=X_{0,\left(\beta_{0}, \ldots, \beta_{l+1}\right)}\right)$.

Proof. From the fact that the function $\tau \mapsto V_{\left(\beta_{0}, \ldots, \beta_{l}, \beta_{l+1}\right)}(\tau$, $\epsilon) e^{-\tau /\left(\epsilon^{r} t\right)}$ is holomorphic on $D\left(0, \rho_{\left(\beta_{0}, \ldots, \beta_{l}, \beta_{l+1}\right)}\right)$, for all $\epsilon \epsilon$ $D\left(0, \epsilon_{0}\right)$, we deduce that its integral along the union of a segment starting from 0 to $\left(\rho_{\left(\beta_{0}, \ldots, \beta_{l+1}\right)} / 2\right) e^{\sqrt{-1} \gamma_{i+1}}$, an arc of circle with radius $\rho_{\left(\beta_{0}, \ldots, \beta_{l+1}\right)} / 2$ connecting $\left(\rho_{\left(\beta_{0}, \ldots, \beta_{l+1}\right)} /\right.$ 2) $e^{\sqrt{-1} \gamma_{i+1}}$ and $\left(\rho_{\left(\beta_{0}, \ldots, \beta_{l+1}\right)} / 2\right) e^{\sqrt{-1} \gamma_{i}}$ and a segment starting from $\left.\left(\rho_{\left(\beta_{0}, \ldots, \beta_{l+1}\right)}\right) 2\right) e^{\sqrt{-1} \gamma_{i}}$ to the origin, is vanishing. Therefore, using property (193), we can write, for all $\left(\beta_{0}, \ldots\right.$, $\left.\beta_{l}, \beta_{l+1}\right) \in \mathbb{N}^{l+2}$,

$$
\begin{aligned}
& X_{i+1,\left(\beta_{0}, \ldots, \beta_{l+1}\right)}(t, \epsilon)-X_{i,\left(\beta_{0}, \ldots, \beta_{l+1}\right)}(t, \epsilon) \\
& =\frac{1}{\epsilon^{r} t}\left(\int_{L_{\rho_{\left(\beta_{0}, \ldots, \beta_{l+1}\right)}, 2, \gamma_{i+1}}} V_{U_{d_{i+1}},\left(\beta_{0}, \ldots, \beta_{l+1}\right)}(\tau, \epsilon) e^{-\tau /\left(\epsilon^{r} t\right)} d \tau\right. \\
& \quad-\int_{L_{\rho_{\left(\beta_{0}, \ldots, \beta_{l+1}\right) / 2}, \gamma_{i}}} V_{U_{d_{i}},\left(\beta_{0}, \ldots, \beta_{l+1}\right)}(\tau, \epsilon) e^{-\tau /\left(\epsilon^{r} t\right)} d \tau \\
& \left.\quad+\int_{\left.C\left(\rho_{\left(\beta_{0}, \ldots, \beta_{l+1}\right)}\right) / 2, \gamma_{i}, \gamma_{i+1}\right)} V_{\left(\beta_{0}, \ldots, \beta_{l+1}\right)}(\tau, \epsilon) e^{-\tau /\left(\epsilon^{r} t\right)} d \tau\right),
\end{aligned}
$$

where

$$
\begin{gathered}
L_{\rho_{\left(\beta_{0}, \ldots, \beta_{l+1}\right)} / 2}, \gamma_{i+1}=\left[\rho_{\left(\beta_{0}, \ldots, \beta_{l+1}\right)} / 2,+\infty\right) e^{\sqrt{-1} \gamma_{i+1}}, \\
L_{\rho_{\left(\beta_{0}, \ldots, \beta_{l+1}\right)}, 2}, \gamma_{i}=\left[\rho_{\left(\beta_{0}, \ldots, \beta_{l+1}\right)} / 2,+\infty\right) e^{\sqrt{-1} \gamma_{i}}
\end{gathered}
$$

and $C\left(\rho_{\left(\beta_{0}, \ldots, \beta_{l+1}\right)} / 2, \gamma_{i}, \gamma_{i+1}\right)$ is an arc of circle centered at 0 with radius $\rho_{\left(\beta_{0}, \ldots, \beta_{l+1}\right)} / 2$ connecting $\left(\rho_{\left(\beta_{0}, \ldots, \beta_{l+1}\right)} / 2\right) e^{\sqrt{-1} \gamma_{i}}$ and $\left(\rho_{\left(\beta_{0}, \ldots, \beta_{l+1}\right)} / 2\right) e^{\sqrt{-1} \gamma_{i+1}}$ for a well-chosen orientation.
First, we give estimates for

$$
\begin{aligned}
& I_{1} \\
& =\left|\frac{1}{\epsilon^{r} t} \int_{L_{\rho_{\left(\beta_{0}, \ldots, \beta_{l+1}\right)}, 2}, \gamma_{i+1}} V_{U_{d_{i+1}},\left(\beta_{0}, \ldots, \beta_{l+1}\right)}(\tau, \epsilon) e^{-\tau /\left(\epsilon^{r} t\right)} d \tau\right| .
\end{aligned}
$$

By construction, the direction $\gamma_{i+1}$ (which depends on $\epsilon^{r} t$ ) is chosen in such a way that $\cos \left(\gamma_{i+1}-\arg \left(\epsilon^{r} t\right)\right) \geq \delta_{1}$ for all $\epsilon \in \mathscr{E}_{i+1} \cap \mathscr{E}_{i}$ and all $t \in \mathscr{T} \cap D\left(0, h^{\prime}\right)$, for some fixed $\delta_{1}>0$. From estimates (192), we deduce that

$$
\begin{aligned}
& I_{1} \leq \frac{1}{\left|\epsilon^{r} t\right|} \int_{\rho_{\left(\beta_{0}, \ldots, \beta_{l+1}\right)} / 2}^{+\infty} C_{20}\left(\frac{l+2}{M^{0}}\right)^{\sum_{j=0}^{l} \beta_{j}} K_{20}^{\beta_{l+1}} \beta_{0} ! \cdots \beta_{l+1} \text { ! } \\
& \cdot e^{\left((\sigma \zeta(b)) /|\epsilon|^{r}\right) h} e^{-\left(h /\left|\epsilon^{r} t\right|\right) \cos \left(\gamma_{i+1}-\arg \left(\epsilon^{r} t\right)\right)} d h \\
& \leq \frac{1}{\left|\epsilon^{r} t\right|} C_{20}\left(\frac{l+2}{M^{0}}\right)^{\sum_{j=0}^{l} \beta_{j}} K_{20}^{\beta_{l+1}} \beta_{0} ! \cdots \beta_{l+1} ! \\
& \cdot \int_{\rho_{\left(\beta_{0}, \ldots, \beta_{l+1}\right)} / 2}^{+\infty} e^{\left(\sigma \zeta(b)-\left(\delta_{1} /|t|\right)\right)\left(h /|\epsilon|^{r}\right)} d h \\
& =C_{20}\left(\frac{l+2}{M^{0}}\right)^{\sum_{j=0}^{l} \beta_{j}} K_{20}^{\beta_{l+1}} \beta_{0} ! \cdots \beta_{l+1} \text { ! } \\
& \cdot \frac{1}{\delta_{1}-\sigma \zeta(b)|t|} e^{-\left(\left(\delta_{1} /|t|\right)-\sigma \zeta(b)\right)\left(\rho_{\left(\beta_{0}, \ldots, \beta_{l+1}\right)} /\left(2|\epsilon|^{r}\right)\right)} \\
& \leq \frac{C_{20}}{\delta_{2}}\left(\frac{l+2}{M^{0}}\right)^{\sum_{j=0}^{l} \beta_{j}} K_{20}^{\beta_{l+1}} \beta_{0} ! \cdots \beta_{l+1} ! \\
& \cdot e^{-\delta_{2}\left(\rho_{\left(\beta_{0}, \ldots, \beta_{l+1}\right)} /\left(2|\epsilon|^{r} h^{\prime}\right)\right)}
\end{aligned}
$$

for all $t \in \mathscr{T} \cap D\left(0, h^{\prime}\right)$ with $|t|<\left(\delta_{1}-\delta_{2}\right) / \sigma \zeta(b)$, for some $0<\delta_{2}<\delta_{1}$ and for all $\epsilon \in \mathscr{E}_{i+1} \cap \mathscr{E}_{i}$.

Now, we provide estimates for

$$
\begin{aligned}
& I_{2} \\
& =\left|\frac{1}{\epsilon^{r} t} \int_{L_{\rho_{\left(\beta_{0}, \ldots, \beta_{l+1}\right)} / 2}, \gamma_{i}} V_{U_{d_{i}},\left(\beta_{0}, \ldots, \beta_{l+1}\right)}(\tau, \epsilon) e^{-\tau /\left(\epsilon^{r} t\right)} d \tau\right| .
\end{aligned}
$$

By construction, the direction $\gamma_{i}$ (wich depends on $\epsilon^{r} t$ ) is chosen in such a way that $\cos \left(\gamma_{i}-\arg \left(\epsilon^{r} t\right)\right) \geq \delta_{1}$ for all $\epsilon \in \mathscr{E}_{i+1} \cap \mathscr{E}_{i}$ and all $t \in \mathscr{T} \cap D\left(0, h^{\prime}\right)$, for some fixed $\delta_{1}>0$. Again, from estimates (192), we deduce as above that

$$
{ }^{I_{2}} \frac{C_{20}}{\delta_{2}}\left(\frac{l+2}{M^{0}}\right)^{\sum_{j=0}^{l} \beta_{j}} K_{20}^{\beta_{l+1}} \beta_{0} ! \cdots \beta_{l+1} ! e^{-\delta_{2}\left(\rho_{\left(\beta_{0}, \ldots, \beta_{l+1}\right)} /\left(2|\epsilon|^{r} h^{\prime}\right)\right)}
$$

for all $t \in \mathscr{T} \cap D\left(0, h^{\prime}\right)$ with $|t|<\left(\delta_{1}-\delta_{2}\right) / \sigma \zeta(b)$, for some $0<\delta_{2}<\delta_{1}$ and for all $\epsilon \in \mathscr{E}_{i+1} \cap \mathscr{E}_{i}$. 
Finally, we give upper bounds for

$$
I_{3}=\left|\int_{C\left(\rho_{\left(\beta_{0}, \ldots, \beta_{l+1}\right)} / 2, \gamma_{i}, \gamma_{i+1}\right)} V_{\left(\beta_{0}, \ldots, \beta_{l+1}\right)}(\tau, \epsilon) e^{-\tau /\left(\epsilon^{r} t\right)} d \tau\right| .
$$

Due to (192) and bearing in mind property (193), we deduce that

$$
\begin{aligned}
I_{3} \leq \frac{1}{\left|\epsilon^{r} t\right|} \mid \int_{\gamma_{i}}^{\gamma_{i+1}} & C_{20}\left(\frac{l+2}{M^{0}}\right)^{\sum_{j=0}^{l} \beta_{j}} K_{20}^{\beta_{l+1}} \beta_{0} ! \cdots \beta_{l+1} ! \\
& \cdot e^{\left((\sigma \zeta(b)) /|\epsilon|^{r}\right)\left(\rho_{\left(\beta_{0}, \ldots, \beta_{l+1}\right)} / 2\right)} \\
& \cdot e^{-\left(\rho_{\left(\beta_{0}, \ldots, \beta_{l+1}\right)} / 2\right)\left(\cos \left(\theta-\arg \left(\epsilon^{r} t\right)\right) /\left|\epsilon^{r} t\right|\right)} \\
& \cdot \frac{\rho_{\left(\beta_{0}, \ldots, \beta_{l+1}\right)}}{2} d \theta \mid .
\end{aligned}
$$

By construction, the circle $C\left(\rho_{\left(\beta_{0}, \ldots, \beta_{l+1}\right)} / 2, \gamma_{i}, \gamma_{i+1}\right)$ is chosen in order that $\cos \left(\theta-\arg \left(\epsilon^{r} t\right)\right) \geq \delta_{1}$ for all $\theta \in\left[\gamma_{i}, \gamma_{i+1}\right]$ (if $\left.\gamma_{i}<\gamma_{i+1}\right)$, for all $\theta \in\left[\gamma_{i+1}, \gamma_{i}\right]$ (if $\gamma_{i+1}<\gamma_{i}$ ), and for every $t \in \mathscr{T} \cap D\left(0, h^{\prime}\right), \epsilon \in \mathscr{E}_{i+1} \cap \mathscr{E}_{i}$. From (202), we get that

$$
\begin{aligned}
I_{3} \leq & \left|\gamma_{i+1}-\gamma_{i}\right| C_{20}\left(\frac{l+2}{M^{0}}\right)^{\sum_{j=0}^{l} \beta_{j}} K_{20}^{\beta_{l+1}} \beta_{0} ! \cdots \beta_{l+1} ! \\
& \cdot \frac{\rho_{\left(\beta_{0}, \ldots, \beta_{l+1}\right)}}{2} \frac{1}{\left|\epsilon^{r} t\right|} e^{-\left(\left(\delta_{1} /|t|\right)-\sigma \zeta(b)\right)\left(\rho_{\left(\beta_{0}, \ldots, \beta_{l+1}\right)} /\left(2|\epsilon|^{r}\right)\right)} \\
\leq & \left|\gamma_{i+1}-\gamma_{i}\right| C_{20}\left(\frac{l+2}{M^{0}}\right)^{\sum_{j=0}^{l} \beta_{j}} K_{20}^{\beta_{l+1}} \beta_{0} ! \cdots \beta_{l+1} ! \\
& \cdot \frac{\rho_{\left(\beta_{0}, \ldots, \beta_{l+1}\right)}}{2} \frac{1}{\left|\epsilon^{r} t\right|} e^{-\left(\delta_{2} / 4\right)\left(\rho_{\left(\beta_{0}, \ldots, \beta_{l+1}\right)} /\left(|\epsilon|^{r}|t|\right)\right)} \\
& \cdot e^{-\left(\delta_{2} / 4\right)\left(\rho_{\left(\beta_{0}, \ldots, \beta_{l+1}\right)} /\left(|\epsilon|^{r} h^{\prime}\right)\right)}
\end{aligned}
$$

for all $t \in \mathscr{T} \cap D\left(0, h^{\prime}\right)$ with $|t|<\left(\delta_{1}-\delta_{2}\right) / \sigma \zeta(b)$, for some $0<\delta_{2}<\delta_{1}$, and for all $\epsilon \in \mathscr{E}_{i+1} \cap \mathscr{E}_{i}$. Regarding inequality (70), we deduce from (203) that

$$
\begin{aligned}
I_{3} \leq & \frac{2 e^{-1}\left|\gamma_{i+1}-\gamma_{i}\right|}{\delta_{2}} C_{20}\left(\frac{l+2}{M^{0}}\right)^{\sum_{j=0}^{l} \beta_{j}} K_{20}^{\beta_{l+1}} \beta_{0} ! \cdots \beta_{l+1} ! \\
& \cdot e^{-\left(\delta_{2} / 4\right)\left(\rho_{\left(\beta_{0}, \ldots, \beta_{l+1}\right)} /\left(|\epsilon|^{r} h^{\prime}\right)\right)}
\end{aligned}
$$

for all $t \in \mathscr{T} \cap D\left(0, h^{\prime}\right)$ with $|t|<\left(\delta_{1}-\delta_{2}\right) / \sigma \zeta(b)$, for some $0<\delta_{2}<\delta_{1}$, and for all $\epsilon \in \mathscr{E}_{i+1} \cap \mathscr{E}_{i}$.

Finally, gathering decomposition (195) and estimates (198), (200), and (204), we get inequality (194).
From Lemma 29 and taking into account assumption (153), we can write

$$
\begin{gathered}
\sup _{t \in \mathscr{T} \cap D\left(0, h^{\prime \prime}\right), z \in H_{\rho_{1}^{\prime}}, x \in D\left(0, \rho_{1}\right)}\left|X_{i+1}(t, z, x, \epsilon)-X_{i}(t, z, x, \epsilon)\right| \\
\leq \sum_{\beta_{0}, \ldots, \beta_{l}, \beta_{l+1} \geq 0} C_{21} e^{-M_{21}\left(\rho_{\left(\beta_{0}, \ldots, \beta_{l+1}\right)} /|\epsilon|^{r}\right)} \\
\cdot\left(\frac{(l+2) e^{\rho_{1}^{\prime}\left(\max _{j=0}^{l}\left|\xi_{j}\right|\right)}}{M^{0}}\right)^{\sum_{j=0}^{l} \beta_{j}}\left(\rho_{1} K_{21}\right)^{\beta_{l+1}} \\
\leq C_{21} \sum_{\beta_{0}, \ldots, \beta_{l}, \beta_{l+1} \geq 0} e^{-M_{21}\left(\rho_{\left(\beta_{0}, \ldots, \beta_{l+1}\right)} /||^{r}\right)} \\
\cdot\left(\max \left\{\rho_{1} K_{21}, \frac{1}{2}\right\}\right)^{\sum_{j=0}^{l+1} \beta_{j}}
\end{gathered}
$$

for all $\epsilon \in \mathscr{E}_{i+1} \cap \mathscr{E}_{i}$ and all $0 \leq i \leq \nu-1$. Moreover, we recall that, for all integers $l \geq 1, \kappa \geq 0$,

$$
\begin{aligned}
\operatorname{Card} & \left\{\left(\beta_{0}, \ldots, \beta_{l+1}\right) \in \frac{\mathbb{N}^{l+2}}{\sum_{j=0}^{l+1} \beta_{j}}=\kappa\right\} \\
& =\frac{(\kappa+l+1) !}{(l+1) ! \kappa !}=\frac{\mathscr{P}_{l}(\kappa)}{(l+1) !},
\end{aligned}
$$

where $\mathscr{P}_{l}(\kappa)=(\kappa+l+1)(\kappa+l) \cdots(\kappa+1)$ is a polynomial of degree $l+1$ in $\kappa$. From definition (89) and with the help of (206), we get two constants $C_{22}>0$ and $0<K_{22}<1$ such that

$$
\begin{gathered}
C_{21} \sum_{\beta_{0}, \ldots, \beta_{l}, \beta_{l+1} \geq 0} e^{-M_{21}\left(\rho_{\left(\beta_{0}, \ldots, \beta_{l+1}\right)} /|\epsilon|^{r}\right)}\left(\max \left\{\rho_{1} K_{21}, \frac{1}{2}\right\}\right)^{\sum_{j=0}^{l+1} \beta_{j}} \\
\leq C_{21} \sum_{\kappa \geq 0} \frac{\mathscr{P}_{l}(\kappa)}{(l+1) !} \exp \left(-M_{21} \frac{C_{\xi_{1}, \ldots, \xi_{l}}^{r_{1} / r_{2}}}{2(1+\kappa)^{h r_{1} / r_{2}}|\epsilon|^{r}}\right) \\
\cdot\left(\max \left\{\rho_{1} K_{21}, \frac{1}{2}\right\}\right)^{\kappa} \\
\leq C_{22} \sum_{\kappa \geq 0} \exp \left(-M_{21} \frac{C_{\xi_{1}, \ldots, \xi_{l}}^{r_{1} / r_{2}}}{2(1+\kappa)^{h r_{1} / r_{2}}|\epsilon|^{r}}\right)\left(K_{22}\right)^{\kappa}
\end{gathered}
$$

for all $\epsilon \in \mathscr{E}_{i+1} \cap \mathscr{E}_{i}$, all $0 \leq i \leq \nu-1$. Now, we recall the following lemma from [4].

Lemma 30. Let $0<a<1$ and $\alpha>0$. There exist $K, M>0$ and $\delta>0$ such that

$$
\sum_{\kappa \geq 0} e^{-\left(1 /(\kappa+1)^{\alpha}\right)(1 / \epsilon)} a^{\kappa} \leq K \exp \left(-M \epsilon^{-1 /(\alpha+1)}\right)
$$

for all $\epsilon \in(0, \delta]$.

From Lemma 30 applied to inequality (207) and from (205), we deduce that estimates (187) hold, if $\epsilon_{0}$ is chosen small enough. Thus proof of Proposition 28 is complete. 
4.2. Existence of Formal Power Series Solutions in the Complex Parameter for the Main Cauchy Problem. This subsection is devoted to explaining the main result of this work. Namely, we will establish the existence of a formal power series

$$
\begin{aligned}
\widehat{X}(t, z, x, \epsilon) & =\sum_{k \geq 0} H_{k}(t, z, x) \frac{\epsilon^{k}}{k !} \\
& \in \mathcal{O}\left(\left(\mathscr{T} \cap D\left(0, h^{\prime \prime}\right)\right) \times H_{\rho_{1}^{\prime}} \times D\left(0, \rho_{1}\right)\right)[[\epsilon]]
\end{aligned}
$$

which solves formally (180) and is constructed in such a way that the actual solutions $X_{i}(t, z, x, \epsilon)$ of the problem (180), (181) all have $\widehat{X}$ as asymptotic expansion of Gevrey order $\left(h r_{1}+r_{2}\right) / r_{3}$ on $\mathscr{E}_{i}$ (as formal series and functions with coefficients and values in the Banach space $\mathcal{O}\left(\left(\mathscr{T} \cap D\left(0, h^{\prime \prime}\right)\right) \times\right.$ $\left.H_{\rho_{1}^{\prime}} \times D\left(0, \rho_{1}\right)\right)$ equipped with the supremum norm) (see Definition 31 below), for all $0 \leq i \leq v-1$.

The proof makes use of a Banach valued version of cohomological criterion for Gevrey asymptotic expansion of sectorial holomorphic functions known in the literature as the Ramis-Sibuya theorem. For a reference, we refer to [18, Section 7.4 Proposition 18] and [30, Lemma XI-2-6].

Definition 31. Let $\left(\mathbb{E},\|\cdot\|_{\mathbb{E}}\right)$ be a complex Banach space over $\mathbb{C}$. One considers a formal series $\widehat{G}(\epsilon)=\sum_{n \geq 0} G_{n} \epsilon^{n}$ where the coefficients $G_{n}$ belong to $\mathbb{E}$ and a holomorphic function $G: \mathscr{E} \rightarrow \mathbb{E}$ on an open bounded sector $\mathscr{E}$ with vertex at 0 . Let $s>0$ be a positive real number. One says that $G$ admits $\widehat{G}$ as its asymptotic expansion of Gevrey order $s$ on $\mathscr{E}$ if, for every proper and bounded subsector $T$ of $\mathscr{E}$, there exist two constants $K, M>0$ such that, for all $N \geq 1$, one has

$$
\left\|G(\epsilon)-\sum_{n=0}^{N-1} G_{n} \epsilon^{n}\right\|_{\mathbb{E}} \leq K M^{N} N !^{s}|\epsilon|^{N}
$$

for all $\epsilon \in T$.

Theorem RS. Let $\left(\mathbb{E},\|\cdot\|_{\mathbb{E}}\right)$ be a complex Banach space over $\mathbb{C}$. Let $\left\{\mathscr{E}_{i}\right\}_{0 \leq i \leq v-1}$ be a good covering in $\mathbb{C}^{*}$. For every $0 \leq i \leq \nu-1$, let $G_{i}$ be a holomorphic function from $\mathscr{E}_{i}$ into $\mathbb{E}$, and let the cocycle $\Delta_{i}(\epsilon):=G_{i+1}(\epsilon)-G_{i}(\epsilon)$ be a holomorphic function from $Z_{i}:=\mathscr{E}_{i} \cap \mathscr{E}_{i+1}$ into $\mathbb{E}$ (with the convention that $\mathscr{E}_{v}=\mathscr{E}_{0}$ and $\left.G_{v}=G_{0}\right)$. We assume that

(1) $G_{i}(\epsilon)$ is bounded as $\epsilon \in \mathscr{E}_{i}$ tends to 0 , for every $0 \leq i \leq$ $v-1$

(2) $\Delta_{i}$ has an exponential decreasing of order $s>0$ on $Z_{i}$, for every $0 \leq i \leq \nu-1$, meaning that there exist $C_{i}, A_{i}>$ 0 such that

$$
\left\|\Delta_{i}(\epsilon)\right\|_{\mathbb{E}} \leq C_{i} e^{-A_{i} /|\epsilon|^{1 / s}},
$$

for every $\epsilon \in Z_{i}$ and $0 \leq i \leq v-1$.

Then, there exists a formal power series $\widehat{G}(\epsilon) \in \mathbb{E}[[\epsilon]]$ such that $G_{i}(\epsilon)$ admits $\widehat{G}(\epsilon)$ as its asymptotic expansion of Gevrey order $s$ on $\mathscr{E}_{i}$, for every $0 \leq i \leq v-1$.

We now state the main result of our paper.
Theorem 32. Let one assume that conditions (185) hold. For all $0 \leq i \leq v-1,0 \leq j \leq S-1$, one also assumes that for the functions $\Xi_{i, j}(t, z, \epsilon)$ constructed in (181) inequalities (186) are fulfilled for some constant I $>0$ given in Proposition 28. Let

$$
\mathbb{E}=\mathcal{O}\left(\left(\mathscr{T} \cap D\left(0, h^{\prime \prime}\right)\right) \times H_{\rho_{1}^{\prime}} \times D\left(0, \rho_{1}\right)\right)
$$

be the Banach space of holomorphic and bounded functions on $\left(\mathscr{T} \cap D\left(0, h^{\prime \prime}\right)\right) \times H_{\rho_{1}^{\prime}} \times D\left(0, \rho_{1}\right)$ equipped with the supremum norm, where $h^{\prime \prime}, \rho_{1}^{\prime}, \rho_{1}$ are the constants appearing in Proposition 28.

Then, there exists a formal series

$$
\widehat{X}(t, z, x, \epsilon)=\sum_{k \geq 0} H_{k}(t, z, x) \frac{\epsilon^{k}}{k !} \in \mathbb{E}[[\epsilon]]
$$

which formally solves the equation

$$
\begin{aligned}
& \left(\epsilon^{r_{3}}\left(t^{2} \partial_{t}+t\right)^{r_{2}}+\left(-i \partial_{z}+1\right)^{r_{1}}\right) \partial_{x}^{S} \widehat{X}(t, z, x, \epsilon) \\
& =\sum_{\left(s, k_{0}, k_{1}, k_{2}\right) \in \mathcal{S}} b_{s, k_{0}, k_{1}, k_{2}}(z, x, \epsilon) t^{s} \partial_{t}^{k_{0}} \partial_{z}^{k_{1}} \partial_{x}^{k_{2}} \widehat{X}(t, z, x, \epsilon) \\
& \quad+\sum_{\left(l_{0}, l_{1}\right) \in \mathcal{N}} c_{l_{0}, l_{1}}(z, x, \epsilon) t^{l_{0}+l_{1}-1}(\widehat{X}(t, z, x, \epsilon))^{l_{1}}
\end{aligned}
$$

and is the Gevrey asymptotic expansion of order $\left(h r_{1}+r_{2}\right) / r_{3}$ of the $\mathbb{E}$-valued function $\epsilon \in \mathscr{E}_{i} \mapsto X_{i}(t, z, x, \epsilon)$, solution of the problem (180), (181) constructed in Proposition 28, for all $0 \leq i \leq v-1$.

Proof. We consider the functions $X_{i}(t, z, x, \epsilon), 0 \leq i \leq \nu-1$ constructed in Proposition 28. For all $0 \leq i \leq v-1$, we define $G_{i}(\epsilon):=(t, z, x) \mapsto X_{i}(t, z, x, \epsilon)$, which is, by construction, a bounded holomorphic function from $\mathscr{E}_{i}$ into the Banach space $\mathbb{E}$ of holomorphic and bounded functions on $(\mathscr{T} \cap$ $\left.D\left(0, h^{\prime \prime}\right)\right) \times H_{\rho_{1}^{\prime}} \times D\left(0, \rho_{1}\right)$ equipped with the supremum norm, where $h^{\prime \prime}, \rho_{1}^{\prime}, \rho_{1}$ are constants appearing in Proposition 28. Bearing in mind estimates (187), we deduce that the cocycle $\Delta_{i}(\epsilon)=G_{i+1}(\epsilon)-G_{i}(\epsilon)$ fulfills estimates of form (211) on $Z_{i}=\mathscr{E}_{i+1} \cap \mathscr{E}_{i}$, where $s=\left(h r_{1}+r_{2}\right) / r_{3}$, for all $0 \leq i \leq$ $v-1$. According to Theorem RS stated above, we deduce the existence of a formal series $\widehat{G}(\epsilon) \in \mathbb{E}[[\epsilon]]$ which is the Gevrey asymptotic expansion of order $\left(h r_{1}+r_{2}\right) / r_{3}$ of $G_{i}(\epsilon)$ on $\mathscr{E}_{i}$, for all $0 \leq i \leq v-1$. Let us define

$$
\widehat{G}(\epsilon)=\widehat{X}(t, z, x, \epsilon)=\sum_{k \geq 0} H_{k}(t, z, x) \frac{\epsilon^{k}}{k !} .
$$

It only remains to show that $\widehat{X}$ is a formal solution of (214). From the fact that $G_{i}(\epsilon)$ admits $\widehat{G}(\epsilon)$ as its asymptotic expansion at 0 on $\mathscr{E}_{i}$, one gets

$$
\begin{aligned}
\lim _{\epsilon \rightarrow 0, \epsilon \in \mathscr{O}_{i}} \sup _{t \in \mathscr{T} \cap D\left(0, h^{\prime \prime}\right), z \in H_{\rho_{1}^{\prime}}, x \in D\left(0, \rho_{1}\right)} \mid \partial_{\epsilon}^{l} X_{i}(t, z, x, \epsilon) \\
-H_{l}(t, z, x) \mid=0
\end{aligned}
$$


for all $l \geq 0$ and all $0 \leq i \leq v-1$. Now, we choose an integer $i \in\{0, \ldots, v-1\}$. By construction, the function $X_{i}(t, z, x, \epsilon)$ solves (180). We differentiate it $l$ times with respect to $\epsilon$. By means of Leibniz's rule, we get that $\partial_{\epsilon}^{l} X_{i}(t, z, x, \epsilon)$ satisfies the identity

$$
\begin{aligned}
& \sum_{h_{1}+h_{2}=l} \frac{l !}{h_{1} ! h_{2} !} \partial_{\epsilon}^{h_{1}}\left(\epsilon^{r_{3}}\right)\left(t^{2} \partial_{t}+t\right)^{r_{2}} \partial_{x}^{S} \partial_{\epsilon}^{h_{2}} X_{i}(t, z, x, \epsilon) \\
& \quad+\left(-i \partial_{z}+1\right)^{r_{1}} \partial_{x}^{S} \partial_{\epsilon}^{l} X_{i}(t, z, x, \epsilon) \\
& =\sum_{\left(s, k_{0}, k_{1}, k_{2}\right) \epsilon \mathcal{S}} t^{s} \\
& \quad \cdot\left(\sum_{h_{1}+h_{2}=l} \frac{l !}{h_{1} ! h_{2} !} \partial_{\epsilon}^{h_{1}} b_{s, k_{0}, k_{1}, k_{2}}(z, x, \epsilon)\right. \\
& \left.+\sum_{\left(l_{0}, l_{1}\right) \epsilon \mathcal{N}} t^{l_{0}+l_{1}-1}\left(\partial_{t}^{k_{0}} \partial_{z}^{k_{1}} \partial_{x}^{k_{2}} \partial_{\epsilon}^{h_{2}} X_{i}\right)(t, z, x, \epsilon)\right) \\
& \quad \sum_{h_{0}+h_{1}+\cdots+h_{l_{1}}=l} \frac{l !}{h_{0} ! \cdots h_{l_{1}} !} \partial_{\epsilon}^{h_{0}} \mathcal{c}_{l_{0}, l_{1}}(z, x, \epsilon) \\
& \quad \prod_{j=1}^{\prod_{1}}\left(\partial_{\epsilon}^{h_{j}} X_{i}\right)(t, z, x, \epsilon)
\end{aligned}
$$

for all $l \geq 1$ and all $(t, z, x, \epsilon) \in\left(\mathscr{T} \cap D\left(0, h^{\prime \prime}\right)\right) \times H_{\rho_{1}^{\prime}} \times D\left(0, \rho_{1}\right) \times$ $\mathscr{E}_{i}$. We let $\epsilon$ tend to 0 in equality (217) and, with the help of (216), we get the following recursions:

$$
\begin{aligned}
& \left(-i \partial_{z}+1\right)^{r_{1}} \partial_{x}^{S} \frac{H_{l}(t, z, x)}{l !} \\
& =\sum_{\left(s, k_{0}, k_{1}, k_{2}\right) \in \mathcal{S}} t^{s} \\
& \quad\left(\sum_{h_{1}+h_{2}=l} \frac{\left(\partial_{\epsilon}^{h_{1}} b_{s, k_{0}, k_{1}, k_{2}}\right)(z, x, 0)}{h_{1} !} \frac{\partial_{t}^{k_{0}} \partial_{z}^{k_{1}} \partial_{x}^{k_{2}} H_{h_{2}}(t, z, x)}{h_{2} !}\right) \\
& +\sum_{\left(l_{0}, l_{1}\right) \in \mathcal{N}} t^{l_{0}+l_{1}-1} \\
& \quad \sum_{h_{0}+h_{1}+\cdots+h_{l_{1}}=l} \frac{\left(\partial_{\epsilon}^{h_{0}} \mathcal{c}_{l_{0}, l_{1}}\right)(z, x, 0)}{h_{0} !} \prod_{j=1}^{l_{1}} \frac{H_{h_{j}}(t, z, x)}{h_{j} !}
\end{aligned}
$$

for all $0 \leq l<r_{3}$, all $(t, z, x) \in\left(\mathscr{T} \cap D\left(0, h^{\prime \prime}\right)\right) \times H_{\rho_{1}^{\prime}} \times D\left(0, \rho_{1}\right)$, and

$$
\begin{aligned}
& \left(t^{2} \partial_{t}+t\right)^{r_{2}} \partial_{x}^{S} \frac{H_{l-r_{3}}(t, z, x)}{\left(l-r_{3}\right) !}+\left(-i \partial_{z}+1\right)^{r_{1}} \partial_{x}^{S} \frac{H_{l}(t, z, x)}{l !} \\
& =\sum_{\left(s, k_{0}, k_{1}, k_{2}\right) \in \mathcal{S}} t^{s}
\end{aligned}
$$

$$
\begin{aligned}
& \cdot\left(\sum_{h_{1}+h_{2}=l} \frac{\left(\partial_{\epsilon}^{h_{1}} b_{s, k_{0}, k_{1}, k_{2}}\right)(z, x, 0)}{h_{1} !} \frac{\partial_{t}^{k_{0}} \partial_{z}^{k_{1}} \partial_{x}^{k_{2}} H_{h_{2}}(t, z, x)}{h_{2} !}\right) \\
& +\sum_{\left(l_{0}, l_{1}\right) \in \mathcal{N}} t^{l_{0}+l_{1}-1} \\
& \cdot \sum_{h_{0}+h_{1}+\cdots+h_{l_{1}}=l} \frac{\left(\partial_{\epsilon}^{h_{0}} c_{l_{0}, l_{1}}\right)(z, x, 0)}{h_{0} !} \prod_{j=1}^{l_{1}} \frac{H_{h_{j}}(t, z, x)}{h_{j} !}
\end{aligned}
$$

for every $l \geq r_{3}$ and all $(t, z, x) \in\left(\mathscr{T} \cap D\left(0, h^{\prime \prime}\right)\right) \times H_{\rho_{1}^{\prime}} \times$ $D\left(0, \rho_{1}\right)$. Since the functions $b_{s, k_{0}, k_{1}, k_{2}}(z, x, \epsilon)$ and $c_{l_{0}, l_{1}}(z, x, \epsilon)$ are analytic with respect to $\epsilon$ near the origin in $\mathbb{C}$, we get that

$$
\begin{aligned}
b_{s, k_{0}, k_{1}, k_{2}}(z, x, \epsilon) & =\sum_{h \geq 0} \frac{\left(\partial_{\epsilon}^{h} b_{s, k_{0}, k_{1}, k_{2}}\right)(z, x, 0)}{h !} \epsilon^{h} \\
c_{l_{0}, l_{1}}(z, x, \epsilon) & =\sum_{h \geq 0} \frac{\left(\partial_{\epsilon}^{h} c_{l_{0}, l_{1}}\right)(z, x, 0)}{h !} \epsilon^{h}
\end{aligned}
$$

for all $\left(s, k_{0}, k_{1}, k_{2}\right) \in \mathcal{S},\left(l_{0}, l_{1}\right) \in \mathcal{N}$ and all $(z, x, \epsilon) \in$ $H_{\rho_{1}^{\prime}} \times D\left(0, \rho_{1}\right) \times D\left(0, \epsilon_{0}\right)$. Finally, gathering recursions (218) and (219) and expansions (220), one can see that the formal series (215) solves (214).

\section{Conflict of Interests}

The authors declare that there is no conflict of interests regarding the publication of this paper.

\section{Acknowledgments}

A. Lastra is partially supported by the Project MTM201231439 of Ministerio de Ciencia e Innovacion, Spain. S. Malek is partially supported by the French ANR-10-JCJC 0105 Project and the PHC Polonium 2013 Project no. 28217SG.

\section{References}

[1] F. Nakajima, "Existence of quasi-periodic solutions of quasiperiodic systems," Funkcialaj Ekvacioj, vol. 15, pp. 61-73, 1972.

[2] T. Mandai, "Existence and nonexistence of null-solutions for some non-Fuchsian partial differential operators with $T$ dependent coefficients," Nagoya Mathematical Journal, vol. 122, pp. 115-137, 1991.

[3] L. Hormander, The Analysis of Linear Partial Differential Operators-I. Distribution Theory and Fourier Analysis, Springer, Berlin, Germany, 2nd edition, 1990.

[4] A. Lastra, S. Malek, and J. Sanz, "On Gevrey solutions of threefold singular nonlinear partial differential equations," Journal of Differential Equations, vol. 255, no. 10, pp. 3205-3232, 2013.

[5] R. Gerard and H. Tahara, Singular Nonlinear Partial Differential Equations, Aspects of Mathematics, Friedrich Vieweg \& Sohn, Brunswick, Germany, 1996.

[6] G. Iooss and A. M. Rucklidge, "On the existence of quasipattern solutions of the Swift-Hohenberg equation," Journal of Nonlinear Science, vol. 20, no. 3, pp. 361-394, 2010. 
[7] L. Eliasson, "Absolutely convergent series expansions for quasiperiodic motions," Mathematical Physics Electronic Journal, vol. 2, no. 4, p. 33, 1996.

[8] G. Gentile, "Quasiperiodic motions in dynamical systems: review of a renormalization group approach," Journal of Mathematical Physics, vol. 51, no. 1, Article ID 015207, 34 pages, 2010.

[9] G. Gentile, M. V. Bartuccelli, and J. H. B. Deane, "Summation of divergent series and Borel summability for strongly dissipative differential equations with periodic or quasiperiodic forcing terms," Journal of Mathematical Physics, vol. 46, no. 6, Article ID 062704, 2005.

[10] M. Bartuccelli and G. Gentile, "Lindstedt series for perturbations of isochronous systems: a review of the general theory," Reviews in Mathematical Physics, vol. 14, no. 2, pp. 121-171, 2002.

[11] O. Costin, G. Gallavotti, G. Gentile, and A. Giuliani, "Borel summability and Lindstedt series," Communications in Mathematical Physics, vol. 269, no. 1, pp. 175-193, 2007.

[12] M. Yamaguchi, "Existence of quasi-periodic solutions of perturbed nonlinear and quasi-linear partial differential equations of standard types," Journal of Mathematical Analysis and Applications, vol. 59, no. 1, pp. 15-28, 1977.

[13] M. Yamaguchi, "Existence of periodic solutions of second order nonlinear evolution equations and applications," Funkcialaj Ekvacioj: Serio Internacia, vol. 38, no. 3, pp. 519-538, 1995.

[14] W. Craig and C. E. Wayne, "Newton's method and periodic solutions of nonlinear wave equations," Communications on Pure and Applied Mathematics, vol. 46, no. 11, pp. 1409-1498, 1993.

[15] S. Kuksin, Nearly Integrable Infinite-Dimensional Hamiltonian Systems, vol. 1556 of Lecture Notes in Mathematics, Springer, Berlin, Germany, 1993.

[16] C. E. Wayne, "Periodic and quasi-periodic solutions of nonlinear wave equations via KAM theory," Communications in Mathematical Physics, vol. 127, no. 3, pp. 479-528, 1990.

[17] S. Malek, "On the summability of formal solutions for doubly singular nonlinear partial differential equations," Journal of Dynamical and Control Systems, vol. 18, no. 1, pp. 45-82, 2012.

[18] W. Balser, Formal Power Series and Linear Systems of Meromorphic Ordinary Differential Equations, Springer, New York, NY, USA, 2000.

[19] B. L. J. Braaksma, "Multisummability of formal power series solutions of nonlinear meromorphic differential equations," Annales de l'Institut Fourier, vol. 42, no. 3, pp. 517-540, 1992.

[20] O. Costin, Asymptotics and Borel Summability, vol. 141 of Chapman \& Hall/CRC Monographs and Surveys in Pure and Applied Mathematics, CRC Press, Boca Raton, Fla, USA, 2009.

[21] J. Ecalle, Les Fonctions Resurgentes, Publications Mathématiques d'Orsay, 1981.

[22] B. Malgrange, "Sommation des series divergentes," Expositiones Mathematicae, vol. 13, no. 2-3, pp. 163-222, 1995.

[23] J.-P. Ramis, “Dévissage Gevrey," in Journées Singulières de Dijon (Univ. Dijon, Dijon, 1978), vol. 59 of Astérisque, pp. 173-204, Société Mathématique de France, Paris, France, 1978.

[24] J. Ramis and Y. Sibuya, "A new proof of multisummability of formal solutions of nonlinear meromorphic differential equations," Annales de l'Institut Fourier, vol. 44, no. 3, pp. 811848, 1994.

[25] D. Damanik and M. Goldstein, "On the existence of global solutions for the KdV equation with quasiperiodic initial data," preprint, 2012.
[26] C. Corduneanu, Almost Periodic Oscillations and Waves, Springer, New York, NY, USA, 2009.

[27] S. Malek, "On singularly perturbed partial integro-differential equations with irregular singularity," Journal of Dynamical and Control Systems, vol. 13, no. 3, pp. 419-449, 2007.

[28] O. Costin and S. Tanveer, "Existence and uniqueness for a class of nonlinear higher-order partial differential equations in the complex plane," Communications on Pure and Applied Mathematics, vol. 53, no. 9, pp. 1092-1117, 2000.

[29] A. Shidlovskii, Transcendental Numbers, vol. 12 of de Gruyter Studies in Mathematics, Walter de Gruyter and Co., Berlin, Germany, 1989, Translated from the Russian by Neal Koblitz. With a foreword by W. Dale Brownawell.

[30] P. Hsieh and Y. Sibuya, Basic Theory of Ordinary Differential Equations, Springer, New York, NY, USA, 1999. 


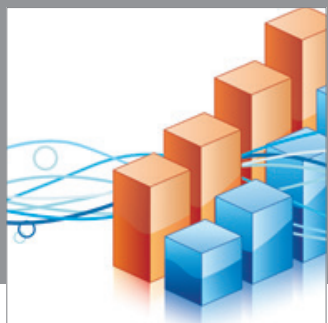

Advances in

Operations Research

mansans

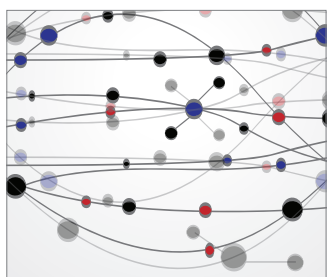

The Scientific World Journal
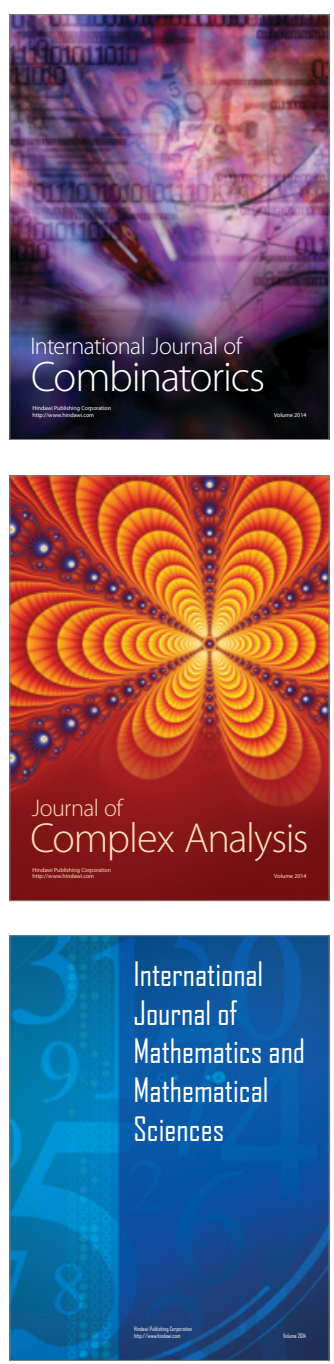
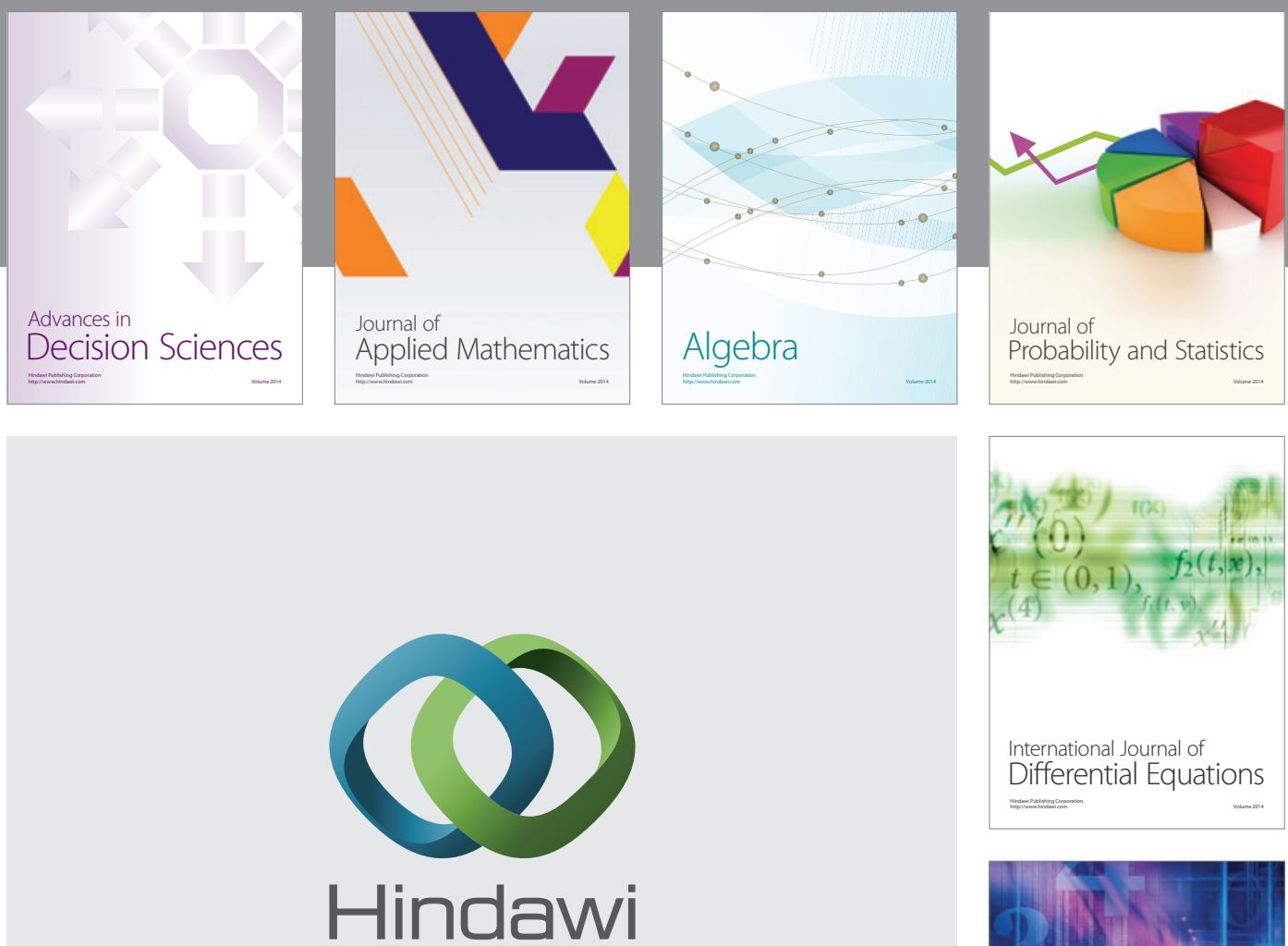

Submit your manuscripts at http://www.hindawi.com
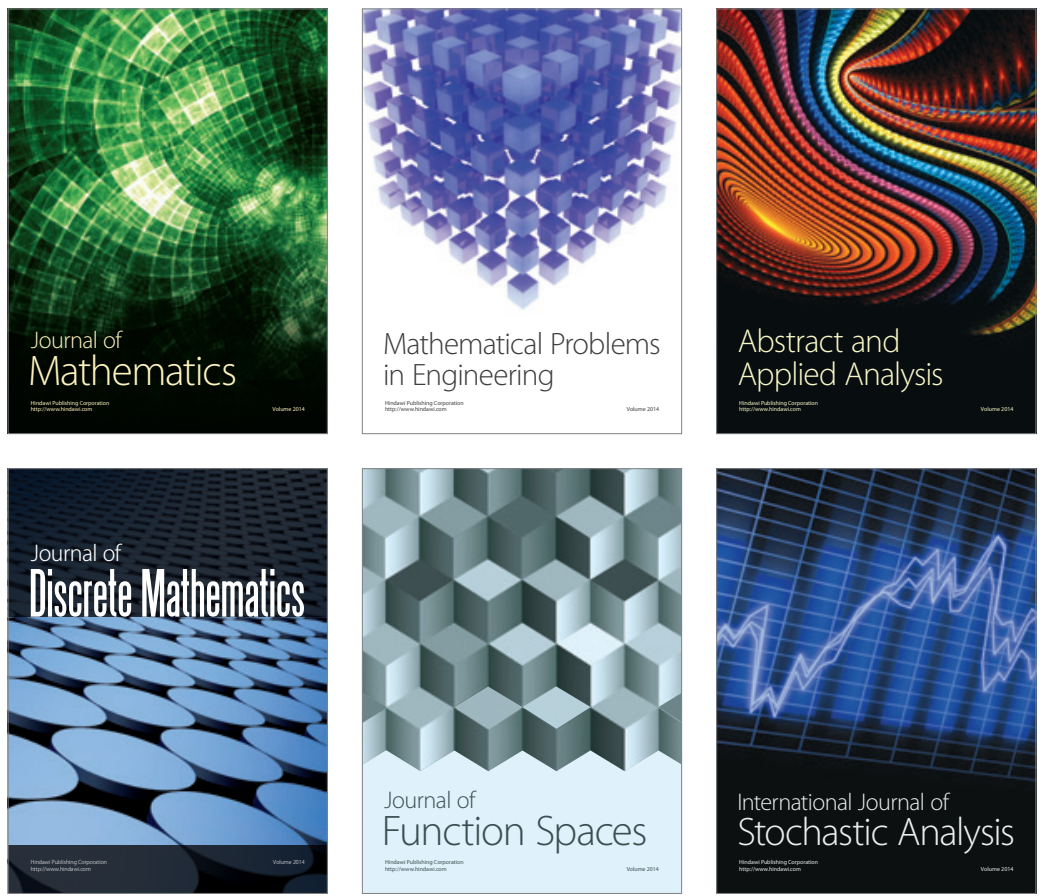

Journal of

Function Spaces

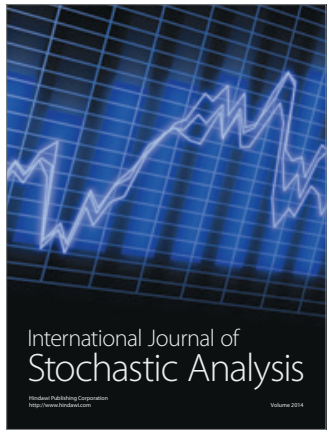

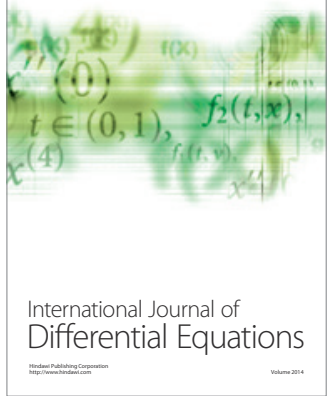
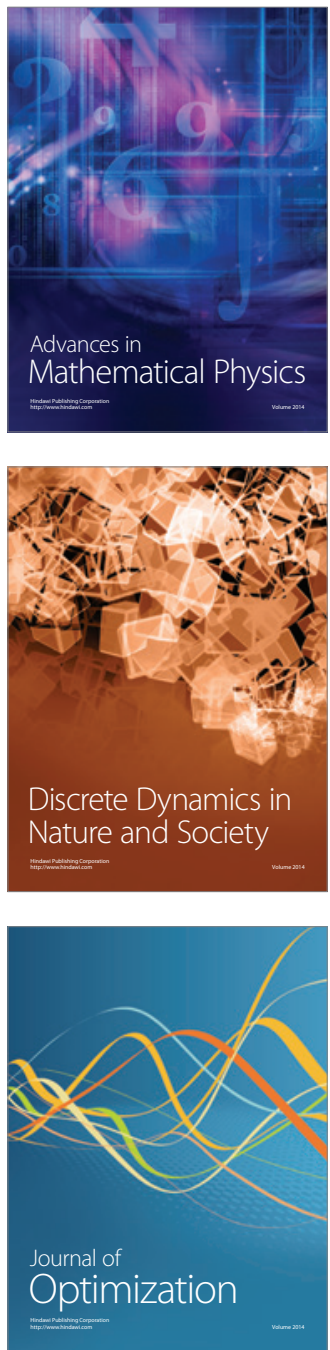